\title{
QUALITATIVE BEHAVIOUR OF INCOMPRESSIBLE TWO-PHASE FLOWS WITH PHASE TRANSITIONS: THE CASE OF NON-EQUAL DENSITIES
}

\author{
JAN PRÜSS, SENJO SHIMIZU, AND MATHIAS WILKE
}

\begin{abstract}
Our study of a basic model for incompressible two-phase flows with phase transitions consistent with thermodynamics in the case of constant but non-equal densities of the phases, begun in [23] is continued. We extend our well-posedness result to general geometries, study the stability of the equilibria of the problem, and show that a solution which does not develop singularities exist globally. And if its limit set contains a stable equilibrium it converges to this equilibrium as time goes to infinity, in the natural state manifold for the problem in an $L_{p}$-setting.
\end{abstract}

\section{Mathematics Subject Classification (2010):}

Primary: 35R35, Secondary: 35Q30, 76D45, 76T10.

Key words: Two-phase Navier-Stokes equations, surface tension, phase transitions, entropy, semiflow, stability, compactness, generalized principle of linearized stability, convergence to equilibria.

Version of March 26, 2018

\section{INTRODUCTION}

Let $\Omega \subset \mathbb{R}^{n}$ be a bounded domain of class $C^{3-}, n \geq 2$. $\Omega$ contains two phases: at time $t$, phase $i$ occupies subdomain $\Omega_{i}(t)$ of $\Omega$. Assume $\partial \Omega_{1}(t) \cap \partial \Omega=\emptyset$; this means no boundary intersection. The closed compact hypersurface $\Gamma(t):=\partial \Omega_{1}(t) \subset \Omega$ forms the interface between the phases.

Let $u$ denote the velocity field, $\pi$ the pressure field, $T(u, \pi, \theta)$ the stress tensor, $D(u)=\left(\nabla u+[\nabla u]^{\top}\right) / 2$ the rate of strain tensor, $\theta$ the (absolute) temperature field, $\nu_{\Gamma}$ the outer normal of $\Omega_{1}, u_{\Gamma}$ the velocity field of the interface, $V_{\Gamma}=u_{\Gamma} \cdot \nu_{\Gamma}$ the normal velocity of $\Gamma(t), H_{\Gamma}=H(\Gamma(t))=-\operatorname{div}_{\Gamma} \nu_{\Gamma}$ the curvature of $\Gamma(t), j$ the phase flux, and $\llbracket v \rrbracket=v_{2}-v_{1}$ the jump of the variable $v$ across $\Gamma(t)$.

S.S. expresses her thanks for hospitality to the Institute of Mathematics, Martin-LutherUniversität Halle-Wittenberg, where important parts of this work originated. The research of S.S was partially supported by JSPS Grant-in-Aid for Scientific Research (B) - 24340025 and Challenging Exploratory Research - 23654048, MEXT. 
Several quantities are derived from the specific free energies $\psi_{i}(\theta)$ as follows. $\epsilon_{i}(\theta):=\psi_{i}(\theta)+\theta \eta_{i}(\theta)$ means the internal energy in phase $i, \eta_{i}(\theta)=-\psi_{i}^{\prime}(\theta)$ the entropy, $\kappa_{i}(\theta):=\epsilon_{i}^{\prime}(\theta)>0$ the heat capacity, and $l(\theta)=\theta \llbracket \psi^{\prime}(\theta) \rrbracket=-\theta \llbracket \eta(\theta) \rrbracket$ the latent heat. Further $d_{i}(\theta)>0$ denotes the coefficient of heat conduction in Fourier's law, $\mu_{i}(\theta)>0$ the viscosity in Newton's law, $\rho_{1}, \rho_{2}>0$ the constant, positive densities of the phases, and $\sigma>0$ the constant coefficient of surface tension. In the sequel we drop the index $i$, as there is no danger of confusion; we just keep in mind that the coefficients depend on the phases.

By an Incompressible Two-Phase Flow with Phase Transition we mean the following problem with sharp interface:

Find a family of closed compact hypersurfaces $\{\Gamma(t)\}_{t \geq 0}$ contained in $\Omega$ and appropriately smooth functions $u: \mathbb{R}_{+} \times \bar{\Omega} \rightarrow \mathbb{R}^{n}$, and $\pi, \theta: \mathbb{R}_{+} \times \bar{\Omega} \rightarrow \mathbb{R}$ such that

$$
\begin{array}{cr}
\rho\left(\partial_{t} u+u \cdot \nabla u\right)-\operatorname{div} T(u, \pi, \theta)=0 & \text { in } \Omega \backslash \Gamma(t), \\
T(u, \pi, \theta)=2 \mu(\theta) D(u)-\pi I, \quad \operatorname{div} u=0 & \text { in } \Omega \backslash \Gamma(t), \\
\llbracket \frac{1}{\rho} \rrbracket j^{2} \nu_{\Gamma}-\llbracket T(u, \pi, \theta) \nu_{\Gamma} \rrbracket=\sigma H_{\Gamma} \nu_{\Gamma} \quad \llbracket u \rrbracket=\llbracket \frac{1}{\rho} \rrbracket j \nu_{\Gamma} & \text { on } \Gamma(t), \\
u=0 \text { on } \partial \Omega, \quad u_{\mid t=0}=u_{0} & \text { in } \Omega \backslash \Gamma(t) . \\
\rho \kappa(\theta)\left(\partial_{t} \theta+u \cdot \nabla \theta\right)-\operatorname{div}(d(\theta) \nabla \theta)-2 \mu|D(u)|_{2}^{2}=0 & \text { in } \Omega \backslash \Gamma(t), \\
\theta \llbracket \eta(\theta) \rrbracket j-\llbracket d(\theta) \partial_{\nu_{\Gamma}} \theta \rrbracket=0, \quad \llbracket \theta \rrbracket=0 & \text { on } \Gamma(t), \\
\partial_{\nu} \theta=0 \text { on } \partial \Omega, \quad \theta_{\mid t=0}=\theta_{0} & \text { in } \Omega . \\
\llbracket \psi(\theta) \rrbracket=-\llbracket \frac{1}{2 \rho^{2}} \rrbracket j^{2}+\llbracket \frac{T(u, \pi, \theta) \nu_{\Gamma} \cdot \nu_{\Gamma}}{\rho} \rrbracket & \text { on } \Gamma(t) . \\
V_{\Gamma}=u_{\Gamma} \cdot \nu_{\Gamma}=u \cdot \nu_{\Gamma}-\frac{1}{\rho} j & \text { on } \Gamma(t), \\
\Gamma_{\mid t=0}=\Gamma_{0} . &
\end{array}
$$

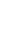


and the total entropy reads

$$
\Phi(\theta, \Gamma)=\int_{\Omega} \rho \eta(\theta) d x .
$$

For the case $\rho_{1} \neq \rho_{2}$ the following result is valid.

Theorem 1.1. Let $\sigma, \rho_{1}, \rho_{2}>0, \rho_{1} \neq \rho_{2}$, and suppose that $\psi_{j} \in C^{3}(0, \infty)$, $\mu_{j}, d_{j} \in C^{2}(0, \infty)$,

$$
-\psi_{j}^{\prime \prime}(s), \mu_{j}(s), d_{j}(s)>0, \quad \text { for } s>0, j=1,2 .
$$

Then the following assertions hold.

(i) The total energy $\mathrm{E}$ is conserved along smooth solutions.

(ii) The negative total entropy $-\Phi$ is a strict Ljapunov functional, which means that $-\Phi$ is strictly decreasing along nonconstant smooth solutions.

(iii) The equilibria without boundary contact are zero velocity, constant temperature, constant pressure in each phase, and $\Omega_{1}$ consists of a finite number of nonintersecting balls of equal size.

(iv) The critical points of the entropy functional with prescribed energy and phase volumes are precisely the equilibria of the system.

(v) Local maxima of the entropy functional with prescribed energy and phase volumes are precisely the equilibria with connected interface.

In equilibrium, the size of the balls follows from conservation of total mass

$$
\rho_{1}\left|\Omega_{1}\right|+\rho_{2}\left|\Omega_{2}\right|=c_{0},
$$

hence

$$
m\left(\omega_{n} / n\right) R^{n}=\left(\rho_{2}|\Omega|-c_{0}\right) / \llbracket \rho \rrbracket ;
$$

once the number of components $m$ of the disperse phase $\Omega_{1}$ is given, this determines the radius $R>0$ of the balls. The temperature is uniquely determined by the total energy

$$
\rho_{1}\left|\Omega_{1}\right| \epsilon_{1}(\theta)+\rho_{2}\left|\Omega_{2}\right| \epsilon_{2}(\theta)+\sigma m \omega_{n} R^{n-1}=\mathrm{E}_{0},
$$

since the functions $\epsilon_{j}(\theta)$ are strictly increasing by assumption. Therefore we have an $n m$-parameter family of equilibria, where the parameters are the centers of the $m$ balls.

It turns out that in case $m=1$ each equilibrium is linearly stable and as one main result in this paper we prove its nonlinear stability. We also prove on the other hand, if $m \geq 2$ then each equilibrium is unstable, linearly as well as nonlinearly; this is a well-known phenomenon called Ostwald ripening in the literature. Thus local maxima of the entropy under the constraints of given energy and phase volumes correspond to the stable equilibria of the system. This is precisely what one would expect physically.

There is a large literature on isothermal incompressible Newtonian two-phase flows without phase transitions, and also on the two-phase Stefan problem with surface tension modeling temperature driven phase transitions. On the other hand, mathematical work on two-phase flow problems including phase transitions are 
rare. In this direction, we only know the papers by Hoffmann and Starovoitov [9, 10, dealing with a simplified two-phase flow model, and Kusaka and Tani [16, 17] which are two-phase for temperature but only one phase is moving. The papers of Di Benedetto and Friedman [5] and Di Benedetto and O'Leary [6] consider weak solutions of conduction-convection problems with phase change. However, none of these papers deals with models which are consistent with thermodynamics.

We emphasize that the major difference between the cases of equal or different densities lies in the occurrence of the so-called Stefan currents which are induced by the jump in the normal velocity across the interface in case $\rho_{1} \neq \rho_{2}$. If the densities are equal these are absent, this case which we call temperature-dominated has been analyzed in 22 and [27. Here we are interested in the velocity-dominated case, i.e. densities $\rho_{1} \neq \rho_{2}$. Note that in this case we may eliminate the phase flux $j$, multiplying the jump condition for $u$ by $\nu_{\Gamma}$, to the result

$$
j=\llbracket u \cdot \nu_{\Gamma} \rrbracket / \llbracket 1 / \rho \rrbracket .
$$

The jump condition for $u$ then becomes $P_{\Gamma} \llbracket u \rrbracket=0$, where $P_{\Gamma}=I-\nu_{\Gamma} \otimes \nu_{\Gamma}$. Similarly, multiplying the second equation in (1.3) with $\rho$ and taking the jump across $\Gamma(t)$ we obtain the new equation

$$
\llbracket \rho \rrbracket V_{\Gamma}=\llbracket \rho u \cdot \nu_{\Gamma} \rrbracket
$$

for the moving interface $\Gamma(t)$. However, it is useful at several occasions to keep the variable $j$, as it has a clear physical meaning.

In 23] the first two authors investigated local well-posedness of this problem in a situation of a nearly flat interface, based on $L_{p}$-maximal regularity of the underlying principal linearization. It is the purpose of this paper to extend this work to general geometries and to present a qualitative analysis of (1.1), (1.2), (1.3) in the framework of $L_{p}$-theory. We study the equilibria of the system which are zero velocities, constant pressures in the phases, constant temperature, and the disperse phase $\Omega_{1}$ consists of a finite number of non-intersecting balls of the same radius. Thus the equilibria form a manifold, and we prove that an equilibrium is normally stable if and only if the phases are connected, otherwise it is normally hyperbolic; see below for a definition of these notions. We further prove by means of the generalized principle of linearized stability that an equilibrium is stable for the nonlinear problem if and only if the phases are connected. Furthermore we show that a solution which does not develop singularities, in a sense specified below, exist globally in the natural state manifold $\mathcal{S} \mathcal{M}$ of the system, and it converges to a probably different equilibrium, provided its limit set in $\mathcal{S M}$ contains a stable equilibrium.

The plan for this paper is as follows. In Section 2 we employ the direct mapping approach to transform the problem locally in time to a fixed domain. This is by now a well established method, we refer in particular to 24] for the necessary geometric background. Section 3 deals with local well-posedness, which is based on maximal $L_{p}$-regularity of the underlying principal linearization and the contraction 
mapping principle. The proof of the crucial maximal regularity result for the arising non-standard asymmetric Stokes-problem is given in Section 7; by perturbation and localization, it is derived from the corresponding result for flat interfaces [23. In Section 4 we study the linearization of the problem at a non-degenerate equilibrium. Employing the generalized principle of linearized stability, in Section 5 we prove these stability assertions also for the nonlinear problem. In Section 6 we introduce the natural state manifold $\mathcal{S M}$ of the system in the $L_{p}$-setting, and it is shown that the local well-posedness result from Section 3 induces a local semiflow on $\mathcal{S M}$. We study its asymptotic behavior, employing the negative entropy as a strict Ljapunov functional, relative compactness of bounded semiorbits and the stability result from Section 5 .

In a subsequent paper we will extend the results of this contribution to the case where the coefficient of surface tension $\sigma>0$ is no longer constant but temperature-dependent. This allows us to include the so-called Marangoni forces.

\section{Transformation to a Fixed Domain}

Let $\Omega \subset \mathbb{R}^{n}$ be a bounded domain with boundary $\partial \Omega$ of class $C^{2}$, and suppose $\Gamma \subset \Omega$ is a hyper-surface of class $C^{2}$, i.e. a $C^{2}$-manifold which is the boundary of a bounded domain $\Omega_{1} \subset \bar{\Omega}_{1} \subset \Omega$; we then set $\Omega_{2}=\Omega \backslash \bar{\Omega}_{1}$. Note that $\Omega_{2}$ is connected, but $\Omega_{1}$ maybe disconnected, however, it consists of finitely many components only, since $\partial \Omega_{1}=\Gamma$ by assumption is a manifold, at least of class $C^{2}$. Recall that the second order bundle of $\Gamma$ is given by

$$
\mathcal{N}^{2} \Gamma:=\left\{\left(p, \nu_{\Gamma}(p), \nabla_{\Gamma} \nu_{\Gamma}(p)\right): p \in \Gamma\right\} .
$$

Here $\nu_{\Gamma}(p)$ denotes the outer normal of $\Omega_{1}$ at $p \in \Gamma$ and $\nabla_{\Gamma}$ the surface gradient on $\Gamma$. The Weingarten tensor $L_{\Gamma}$ on $\Gamma$ is defined by

$$
L_{\Gamma}(p)=-\nabla_{\Gamma} \nu_{\Gamma}(p), \quad p \in \Gamma .
$$

The eigenvalues $\kappa_{j}(p)$ of $L_{\Gamma}(p)$ are the principal curvatures of $\Gamma$ at $p \in \Gamma$, and we have $\left|L_{\Gamma}(p)\right|=\max _{j}\left|\kappa_{j}(p)\right|$. The curvature $H_{\Gamma}(p)$ (more precisely $(n-1)$ times mean curvature) is defined as the trace of $L_{\Gamma}(p)$, i.e.

$$
H_{\Gamma}(p)=\sum_{j=1}^{n-1} \kappa_{j}=-\operatorname{div}_{\Gamma} \nu_{\Gamma}(p),
$$

where $\operatorname{div}_{\Gamma}$ means surface divergence. Recall also the Haussdorff distance $d_{H}$ between the two closed subsets $A, B \subset \mathbb{R}^{m}$, defined by

$$
d_{H}(A, B):=\max \left\{\sup _{a \in A} \operatorname{dist}(a, B), \sup _{b \in B} \operatorname{dist}(b, A)\right\} .
$$

Then we may approximate $\Gamma$ by a real analytic hyper-surface $\Sigma$ (or merely $\Sigma \in C^{3}$ ), in the sense that the Haussdorff distance of the second order bundles of $\Gamma$ and $\Sigma$ is as small as we want. More precisely, for each $\eta>0$ there is a real analytic closed hyper-surface $\Sigma$ such that $d_{H}\left(\mathcal{N}^{2} \Sigma, \mathcal{N}^{2} \Gamma\right) \leq \eta$. If $\eta>0$ is small enough, then $\Sigma$ bounds a domain $\Omega_{1}^{\Sigma}$ with $\overline{\Omega_{1}^{\Sigma}} \subset \Omega$, and we set $\Omega_{2}^{\Sigma}=\Omega \backslash \overline{\Omega_{1}^{\Sigma}}$. 
It is well known that a hyper-surface $\Sigma$ of class $C^{2}$ admits a tubular neighborhood, which means that there is $a>0$ such that the map

$$
\begin{aligned}
& \Lambda: \Sigma \times(-a, a) \rightarrow \mathbb{R}^{n} \\
& \Lambda(p, r):=p+r \nu_{\Sigma}(p)
\end{aligned}
$$

is a diffeomorphism from $\Sigma \times(-a, a)$ onto $\mathcal{R}(\Lambda)$, where $\nu_{\Sigma}(p)$ denotes the outer normal at $p \in \Sigma$. The inverse

$$
\Lambda^{-1}: \mathcal{R}(\Lambda) \mapsto \Sigma \times(-a, a)
$$

of this map is conveniently decomposed as

$$
\Lambda^{-1}(x)=\left(\Pi_{\Sigma}(x), d_{\Sigma}(x)\right), \quad x \in \mathcal{R}(\Lambda) .
$$

Here $\Pi_{\Sigma}(x)$ means the orthogonal projection of $x$ to $\Sigma$ and $d_{\Sigma}(x)$ the signed distance from $x$ to $\Sigma$; so $\left|d_{\Sigma}(x)\right|=\operatorname{dist}(x, \Sigma)$ and $d_{\Sigma}(x)<0$ if and only if $x \in \Omega_{1}^{\Sigma}$. In particular we have $\mathcal{R}(\Lambda)=\left\{x \in \mathbb{R}^{n}: \operatorname{dist}(x, \Sigma)<a\right\}$.

Note that on the one hand, $a$ is determined by the curvatures of $\Sigma$, i.e. we must have

$$
0<a<\min \left\{1 /\left|\kappa_{j}(p)\right|: j=1, \ldots, n-1, p \in \Sigma\right\},
$$

where $\kappa_{j}(p)$ mean the principal curvatures of $\Sigma$ at $p \in \Sigma$. But on the other hand, $a$ is also connected to the topology of $\Sigma$, which can be expressed as follows. Since $\Sigma$ is a compact manifold of dimension $n-1$ it satisfies the ball condition, which means that there is a radius $r_{\Sigma}>0$ such that for each point $p \in \Sigma$ there are $x_{j} \in \Omega_{j}^{\Sigma}, j=1,2$, such that $B_{r_{\Sigma}}\left(x_{j}\right) \subset \Omega_{j}^{\Sigma}$, and $\bar{B}_{r_{\Sigma}}\left(x_{j}\right) \cap \Sigma=\{p\}$. Choosing $r_{\Sigma}$ maximal, we then must also have $a<r_{\Sigma}$. Note that $1 / r_{\Sigma}$ also bounds the principal curvatures, we have

$$
\left|\kappa_{j}(p)\right| \leq 1 / r_{\Sigma}, \quad j=1, \ldots, n-1, p \in \Sigma .
$$

In the sequel we fix $a=r_{\Sigma} / 2$.

For later use we note that the derivatives of $\Pi_{\Sigma}(x)$ and $d_{\Sigma}(x)$ are given by

$$
\nabla d_{\Sigma}(x)=\nu_{\Sigma}\left(\Pi_{\Sigma}(x)\right), \quad D(u) \Pi_{\Sigma}(x)=M_{0}\left(d_{\Sigma}(x)\right)\left(\Pi_{\Sigma}(x)\right) P_{\Sigma}\left(\Pi_{\Sigma}(x)\right),
$$

where $P_{\Sigma}(p)=I-\nu_{\Sigma}(p) \otimes \nu_{\Sigma}(p)$ denotes the orthogonal projection onto the tangent space $T_{p} \Sigma$ of $\Sigma$ at $p \in \Sigma$, and $M_{0}(r)(p)=\left(I-r L_{\Sigma}(p)\right)^{-1}$. Note that

$$
\left|M_{0}(r)(p)\right| \leq 1 /\left(1-r\left|L_{\Sigma}(p)\right|\right) \leq 2, \quad \text { for all } p \in \Sigma,|r| \leq a .
$$

All of these facts are discussed in more detail in 24 .

Setting $\Gamma=\Gamma(t)$, we may use the map $\Lambda$ to parameterize the unknown free boundary $\Gamma(t)$ over $\Sigma$ by means of a height function $h(t, p)$ via

$$
\Gamma(t): p \mapsto p+h(t, p) \nu_{\Sigma}(p), \quad p \in \Sigma,
$$

for small $t \geq 0$, at least. Extend this diffeomorphism to all of $\bar{\Omega}$ by means of

$$
\Xi_{h}(t, x)=x+\chi\left(d_{\Sigma}(x) / a\right) h\left(t, \Pi_{\Sigma}(x)\right) \nu_{\Sigma}\left(\Pi_{\Sigma}(x)\right)=: x+\xi_{h}(t, x) .
$$


Here $\chi$ denotes a suitable cut-off function; more precisely, $\chi \in \mathcal{D}(\mathbb{R}), 0 \leq \chi \leq 1$, $\chi(r)=1$ for $|r|<1 / 3$, and $\chi(r)=0$ for $|r|>2 / 3$. Note that $\Xi_{h}(t, x)=x$ for $\left|d_{\Sigma}(x)\right|>2 a / 3$, and

$$
\Xi_{h}^{-1}(t, p)=p-h(t, p) \nu_{\Sigma}(p) \quad \text { for } p \in \Sigma .
$$

Now we define the transformed quantities

$$
\begin{array}{ll}
\bar{u}(t, x)=u\left(t, \Xi_{h}(t, x)\right), & \bar{\theta}(t, x)=\theta\left(t, \Xi_{h}(t, x)\right), \\
\bar{j}(t, x)=j\left(t, \Xi_{h}(t, x)\right), & \bar{\pi}(t, x)=\pi\left(t, \Xi_{h}(t, x)\right), \quad t>0, x \in \Omega \backslash \Sigma,
\end{array}
$$

the pull backs of $u, \theta, j$, and $\pi$. This way we have transformed the time varying regions $\Omega \backslash \Gamma(t)$ to the fixed domain $\Omega \backslash \Sigma$.

This transformation gives the following problem for $\bar{u}, \bar{\pi}, \bar{\theta}, \bar{j}, h$.

$$
\begin{aligned}
& \rho \partial_{t} \bar{u}-\mathcal{G}(h) \cdot \mu(\bar{\theta})\left(\mathcal{G}(h) \bar{u}+[\mathcal{G}(h) \bar{u}]^{\top}\right)+\mathcal{G}(h) \bar{\pi}=\mathcal{R}_{u}(\bar{u}, \bar{\theta}, h) \quad \text { in } \Omega \backslash \Sigma, \\
& \mathcal{G}(h) \cdot \bar{u}=0 \quad \text { in } \Omega \backslash \Sigma, \\
& \rho \kappa(\bar{\theta}) \partial_{t} \bar{\theta}-\mathcal{G}(h) \cdot d(\bar{\theta}) \mathcal{G}(h) \bar{\theta}=\mathcal{R}_{\theta}(\bar{u}, \bar{\theta}, h) \quad \text { in } \Omega \backslash \Sigma, \\
& \bar{u}=\partial_{\nu} \bar{\theta}=0 \quad \text { on } \partial \Omega \text {, } \\
& \llbracket 1 / \rho \rrbracket \bar{j}^{2} \nu_{\Gamma}(h)+\llbracket-\mu(\bar{\theta})\left(\mathcal{G}(h) \bar{u}+[\mathcal{G}(h) \bar{u}]^{\top}\right)+\bar{\pi} \rrbracket \nu_{\Gamma}(h)=\sigma H_{\Gamma}(h) \nu_{\Gamma}(h) \text { on } \Sigma, \\
& P_{\Gamma} \llbracket \bar{u} \rrbracket=0, \quad \bar{j}=\llbracket u \cdot \nu_{\Gamma} \rrbracket / \llbracket 1 / \rho \rrbracket, \quad \llbracket \bar{\theta} \rrbracket=0 \quad \text { on } \Sigma, \\
& l(\bar{\theta}) \bar{j}+\llbracket d(\bar{\theta}) \mathcal{G}(h) \bar{\theta} \cdot \nu_{\Gamma}(h) \rrbracket=0, \quad \text { on } \Sigma, \\
& \llbracket \psi(\bar{\theta}) \rrbracket+\llbracket 1 / 2 \rho^{2} \rrbracket \bar{j}^{2}-\llbracket-(\mu(\bar{\theta}) / \rho)\left(\mathcal{G}(h) \bar{u}+\left[\mathcal{G}(h) \bar{u} \rrbracket^{\top}\right)-\bar{\pi} / \rho \rrbracket \nu_{\Gamma}(h)=0 \quad \text { on } \Sigma,\right. \\
& \llbracket \rho \rrbracket V_{\Gamma}-\llbracket \rho \bar{u} \cdot \nu_{\Gamma} \rrbracket=0, \quad \text { on } \Sigma, \\
& \bar{u}(0)=\bar{u}_{0}, \quad \bar{\theta}(0)=\bar{\theta}_{0}, \quad h(0)=h_{0} .
\end{aligned}
$$

Here $\mathcal{G}(h)$ and $H_{\Gamma}(h)$ denote the transformed gradient and curvature, respectively. More precisely, we have

$$
D \Xi_{h}=I+D \xi_{h}, \quad\left[D \Xi_{h}\right]^{-1}=I-\left[I+D \xi_{h}\right]^{-1} D \xi_{h}=I-M_{1}(h)^{\top} .
$$

A simple computation yields

$$
\begin{aligned}
D \xi_{h}(t, x) & =\nu_{\Sigma}\left(\Pi_{\Sigma}(x)\right) \otimes M_{0}\left(d_{\Sigma}(x)\right)\left(\Pi_{\Sigma}(x)\right) \nabla_{\Sigma} h\left(t, \Pi_{\Sigma}(x)\right) \\
& -h\left(t, \Pi_{\Sigma}(x)\right) L_{\Sigma}\left(\Pi_{\Sigma}(x)\right) M_{0}\left(d_{\Sigma}(x)\right)\left(\Pi_{\Sigma}(x)\right) P_{\Sigma}\left(\Pi_{\Sigma}(x)\right)
\end{aligned}
$$

for $\left|d_{\Sigma}(x)\right|<a / 3, D \xi_{h}(t, x)=0$ for $\left|d_{\Sigma}(x)\right|>2 a / 3$, as well as

$$
\begin{aligned}
D \xi_{h}(t, x)= & \frac{1}{a} \chi^{\prime}\left(d_{\Sigma}(x) / a\right) h\left(t, \Pi_{\Sigma}(x)\right) \nu_{\Sigma}\left(\Pi_{\Sigma}(x)\right) \otimes \nu_{\Sigma}\left(\Pi_{\Sigma}(x)\right) \\
+ & \chi\left(d_{\Sigma}(x) / a\right)\left[\nu_{\Sigma}\left(\Pi_{\Sigma}(x)\right) \otimes M_{0}\left(d_{\Sigma}(x)\right)\left(\Pi_{\Sigma}(x)\right) \nabla_{\Sigma} h\left(t, \Pi_{\Sigma}(x)\right)\right. \\
& \left.-h\left(t, \Pi_{\Sigma}(x)\right) L_{\Sigma}\left(\Pi_{\Sigma}(x)\right) M_{0}\left(d_{\Sigma}(x)\right)\left(\Pi_{\Sigma}(x)\right) P_{\Sigma}\left(\Pi_{\Sigma}(x)\right)\right] \\
& \quad \text { for } a / 3<\left|d_{\Sigma}(x)\right|<2 a / 3,
\end{aligned}
$$

Therefore $\left[I+D \xi_{h}\right]$ is invertible, provided $h$ and $\nabla_{\Sigma} h$ are sufficiently small, more precisely

$$
\left|\left[I+D \xi_{h}\right]^{-1}\right| \leq 2, \text { for }|h|_{\infty}<\frac{1}{10} \min \left\{a /\left|\chi^{\prime}\right|_{\infty}, 1 /\left|L_{\Sigma}\right|_{\infty}\right\},\left|\nabla_{\Sigma} h\right|_{\infty}<\frac{1}{10} .
$$


With these properties we get

$$
\begin{aligned}
{[\nabla \pi] \circ \Xi_{h} } & =\mathcal{G}(h) \bar{\pi} \\
& =\left(\left[D \Xi_{h}\right]^{-\mathrm{\top}} \circ \Xi_{h}\right) \nabla \bar{\pi}=\nabla \bar{\pi}-\left[D \xi_{h}\right]^{\mathrm{\top}}\left[I+D \xi_{h}\right]^{-\mathrm{T}} \nabla \bar{\pi} \\
& =:\left(I-M_{1}(h)\right) \nabla \bar{\pi} \\
{[\nabla \theta] \circ \Xi_{h} } & =\left(I-M_{1}(h)\right) \nabla \bar{\theta} \\
\operatorname{div} u \circ \Xi_{h} & =\mathcal{G}(h) \cdot \bar{u}=\left(I-M_{1}(h)\right) \nabla \cdot \bar{u} .
\end{aligned}
$$

Next we note that

$$
\begin{aligned}
\partial_{t} u \circ \Xi_{h} & =\partial_{t} \bar{u}-[\nabla u] \circ \Xi_{h} \cdot \partial_{t} \Xi_{h}=\partial_{t} \bar{u}-\left(\left[D \Xi_{h}\right]^{-\mathrm{T}} \circ \Xi_{h}\right) \nabla \bar{u} \cdot \partial_{t} \Xi_{h} \\
& =\partial_{t} \bar{u}-\nabla \bar{u} \cdot\left[I+D \xi_{h}\right]^{-1} \partial_{t} \xi_{h}=: \partial_{t} \bar{u}-(R(h) \cdot \nabla) \bar{u},
\end{aligned}
$$

with $R(h)=\left(I-M_{1}(h)^{\boldsymbol{\top}}\right) \partial_{t} \xi_{h}$. This yields

$$
\mathcal{R}_{u}(\bar{u}, \bar{\theta}, h)=-\rho \bar{u} \cdot \mathcal{G}(h) \bar{u}+\rho(R(h) \cdot \nabla) \bar{u},
$$

and

$$
\begin{aligned}
\mathcal{R}_{\theta}(\bar{u}, \bar{\theta}, h) & =-\rho \kappa(\bar{\theta}) \bar{u} \cdot \mathcal{G}(h) \bar{\theta}+\rho \kappa(\bar{\theta})(R(h) \cdot \nabla) \bar{\theta} \\
& +\mu(\bar{\theta})\left(\mathcal{G}(h) \bar{u}+[\mathcal{G}(h) \bar{u}]^{\top}\right) \cdot \mathcal{G}(h) \bar{u} .
\end{aligned}
$$

With the Weingarten tensor $L_{\Sigma}$ and the surface gradient $\nabla_{\Sigma}$ we further have

$$
\begin{aligned}
\nu_{\Gamma}(h)=\beta(h)\left(\nu_{\Sigma}-\alpha(h)\right), & \alpha(h) & =M_{0}(h) \nabla_{\Sigma} h, \\
M_{0}(h)=\left(I-h L_{\Sigma}\right)^{-1}, & \beta(h) & =\left(1+|\alpha(h)|^{2}\right)^{-1 / 2},
\end{aligned}
$$

and

$$
V_{\Gamma}=\left(\partial_{t} \Xi \mid \nu_{\Gamma}\right)=\partial_{t} h\left(\nu_{\Gamma} \mid \nu_{\Sigma}\right)=\beta(h) \partial_{t} h .
$$

The curvature $H_{\Gamma}(h)$ becomes

$$
H_{\Gamma}(h)=\beta(h)\left\{\operatorname{tr}\left[M_{0}(h)\left(L_{\Sigma}+\nabla_{\Sigma} \alpha(h)\right)\right]-\beta^{2}(h)\left(M_{0}(h) \alpha(h) \mid\left[\nabla_{\Sigma} \alpha(h)\right] \alpha(h)\right)\right\},
$$

a differential expression involving second order derivatives of $h$ only linearly. Its linearization at $h=0$ is given by

$$
H_{\Gamma}^{\prime}(0)=\operatorname{tr} L_{\Sigma}^{2}+\Delta_{\Sigma}
$$

Here $\Delta_{\Sigma}$ denotes the Laplace-Beltrami operator on $\Sigma$.

It is convenient to decompose the stress boundary condition into tangential and normal parts. Multiplying the stress interface condition with $\nu_{\Sigma} / \beta$ we obtain

$$
\llbracket 1 / \rho \rrbracket \bar{j}^{2}+\llbracket \bar{\pi} \rrbracket-\sigma H_{\Gamma}(h)=\left(\llbracket \mu(\bar{\theta})\left(\mathcal{G}(h) \bar{u}+\left[\mathcal{G}(h) \bar{u} \rrbracket^{\top}\right) \rrbracket\left(\nu_{\Sigma}-M_{0}(h) \nabla_{\Sigma} h\right) \mid \nu_{\Sigma}\right),\right.
$$

for the normal part of the stress boundary condition, and

$$
\begin{aligned}
& -P_{\Sigma} \llbracket \mu(\bar{\theta})\left(\mathcal{G}(h) \bar{u}+[\mathcal{G}(h) \bar{u}]^{\top}\right) \rrbracket\left(\nu_{\Sigma}-M_{0}(h) \nabla_{\Sigma} h\right) \\
& \quad=\left(\llbracket \mu(\bar{\theta})\left(\mathcal{G}(h) \bar{u}+\left[\mathcal{G}(h) \bar{u} \rrbracket^{\top}\right) \rrbracket\left(\nu_{\Sigma}-M_{0}(h) \nabla_{\Sigma} h\right) \mid \nu_{\Sigma}\right) M_{0}(h) \nabla_{\Sigma} h,\right.
\end{aligned}
$$

for the tangential part. Note that the latter neither contains the pressure jump nor the phase flux nor the curvature, which is the advantage of this decomposition. 


\section{Local WeLl-Posedness}

In this section we prove local well-posedness of problem (1.1), (1.2), (1.3). The proof is based on maximal $L_{p}$-regularity of the principal linearization and on the contraction mapping principle.

3.1. Principal Linearization. The principal part of the linearized problem reads as follows

$$
\begin{array}{rlrl}
\rho \partial_{t} u-\mu(x) \Delta u+\nabla \pi & =\rho f_{u} & \text { in } \Omega \backslash \Sigma, \\
\operatorname{div} u & =g_{d} \quad & \text { in } \Omega \backslash \Sigma, \\
P_{\Sigma} \llbracket u \rrbracket+c(t, x) \nabla_{\Sigma} h & =P_{\Sigma} g_{u} \quad & \text { on } \Sigma, \\
u & =0 \quad \text { on } \partial \Omega \\
-2 \llbracket \mu(x) D(u) \nu_{\Sigma} \rrbracket+\llbracket \pi \rrbracket \nu_{\Sigma}-\sigma \Delta_{\Sigma} h \nu_{\Sigma} & =g \quad \text { on } \Sigma, \\
u(0) & =u_{0} \quad \text { in } \Omega \backslash \Sigma . \\
\rho \kappa(x) \partial_{t} \theta-d(x) \Delta \theta & =\rho \kappa(x) f_{\theta} \quad \text { in } \Omega \backslash \Sigma, \\
-\llbracket d(x) \partial_{\nu_{\Sigma}} \theta \rrbracket & =g_{\theta} \quad \text { on } \Sigma, & \\
\llbracket \theta \rrbracket & =0 \quad \text { on } \Sigma, & \\
\partial_{\nu} \theta & =0 \quad \text { on } \partial \Omega \\
\theta(0) & =\theta_{0} \quad \text { in } \Omega . & \\
\llbracket \rho \rrbracket \partial_{t} h-\llbracket \rho u \cdot \nu_{\Sigma} \rrbracket+b(t, x) \cdot \nabla_{\Sigma} h & =\llbracket \rho \rrbracket f_{h} \quad \text { on } \Sigma, \\
-2 \llbracket(\mu(x) / \rho) D(u) \nu_{\Sigma} \cdot \nu_{\Sigma} \rrbracket+\llbracket \pi / \rho \rrbracket & =g_{h} \quad \text { on } \Sigma, \\
h(0) & =h_{0} \quad \text { on } \Sigma .
\end{array}
$$

Here $\mu_{k}, \kappa_{k}, d_{k}, k=1,2$, are functions of $x$, continuous on $\bar{\Omega}_{k}$, and $c, b$ depend on $t$ and $x$; recall that $\llbracket \rho \rrbracket \neq 0$ by assumption. Apparently, (3.2) decouples from the remaining problem. Since it is well-known that this problem has maximal $L_{p}$-regularity, we concentrate on the remaining one for $(u, \pi, h)$.

$$
\begin{aligned}
\rho \partial_{t} u-\mu(x) \Delta u+\nabla \pi & =\rho f_{u} \quad \text { in } \Omega \backslash \Sigma, \\
\operatorname{div} u & =g_{d} \quad \text { in } \Omega \backslash \Sigma, \\
u & =0 \quad \text { on } \partial \Omega \\
P_{\Sigma} \llbracket u \rrbracket+c(t, x) \nabla_{\Sigma} h & =P_{\Sigma} g_{u} \quad \text { on } \Sigma, \\
-2 \llbracket \mu(x) D(u) \nu_{\Sigma} \rrbracket+\llbracket \pi \rrbracket \nu_{\Sigma}-\sigma \Delta_{\Sigma} h \nu_{\Sigma} & =g \quad \text { on } \Sigma, \\
-2 \llbracket \mu(x) D(u) \nu_{\Sigma} \cdot \nu_{\Sigma} / \rho \rrbracket+\llbracket \pi / \rho \rrbracket & =g_{h} \quad \text { on } \Sigma, \\
\llbracket \rho \rrbracket \partial_{t} h-\llbracket \rho u \cdot \nu_{\Sigma} \rrbracket+b(t, x) \cdot \nabla_{\Sigma} h & =\llbracket \rho \rrbracket f_{h} \quad \text { on } \Sigma, \\
u(0) & =u_{0} \quad \text { in } \Omega \backslash \Sigma, \\
h(0) & =h_{0} \quad \text { on } \Sigma .
\end{aligned}
$$

For this problem we have maximal regularity result in the $L_{p}$-setting. 
Theorem 3.1. Let $p>n+2$ be fixed, $\rho_{j}>0, \rho_{2} \neq \rho_{1}, \mu_{j} \in C\left(\bar{\Omega}_{j}\right), \mu_{j}>0$, $j=1,2,(b, c) \in\left[W_{p}^{1-1 / 2 p}\left(J ; L_{p}(\Sigma)\right) \cap L_{p}\left(J ; W_{p}^{2-1 / p}(\Sigma)\right)\right]^{n+1}$, where $J=[0, a]$.

Then the Stokes problem with free boundary (3.4) admits a unique solution $(u, \pi, h)$ with regularity

$$
\begin{aligned}
& u \in H_{p}^{1}\left(J ; L_{p}(\Omega)\right)^{n} \cap L_{p}\left(J ; H_{p}^{2}(\Omega \backslash \Sigma)\right)^{n}, \\
& \llbracket u \cdot \nu_{\Sigma} \rrbracket \in H_{p}^{1}\left(J ; \dot{W}_{p}^{-1 / p}(\Sigma)\right), \quad \pi \in L_{p}\left(J ; \dot{H}_{p}^{1}(\Omega \backslash \Sigma)\right), \\
& \pi_{j}:=\pi_{\mid \partial \Omega_{j} \cap \Sigma} \in W_{p}^{1 / 2-1 / 2 p}\left(J ; L_{p}(\Sigma)\right) \cap L_{p}\left(J ; W_{p}^{1-1 / p}(\Sigma)\right), j=1,2, \\
& h \in W_{p}^{2-1 / 2 p}\left(J ; L_{p}(\Sigma)\right) \cap H_{p}^{1}\left(J ; W_{p}^{2-1 / p}(\Sigma)\right) \cap L_{p}\left(J ; W_{p}^{3-1 / p}(\Sigma)\right)
\end{aligned}
$$

if and only if the data $f_{u}, g_{d}, g, P_{\Sigma} g_{u}, g_{h}, f_{h}, u_{0}, h_{0}$ satisfy the following regularity and compatibility conditions:

(a) $f_{u} \in L_{p}\left(J ; L_{p}\left(\Omega, \mathbb{R}^{n+1}\right)\right)$,

(b) $g_{d} \in H_{p}^{1}\left(J ; \dot{H}_{p}^{-1}(\Omega)\right) \cap L_{p}\left(J ; H_{p}^{1}(\Omega \backslash \Sigma)\right)$,

(c) $\left(g, g_{h}\right) \in W_{p}^{1 / 2-1 / 2 p}\left(J ; L_{p}\left(\Sigma, \mathbb{R}^{n+1}\right)\right) \cap L_{p}\left(J ; W_{p}^{1-1 / p}\left(\Sigma, \mathbb{R}^{n+1}\right)\right)$,

(d) $\left(P_{\Sigma} g_{u}, f_{h}\right) \in W_{p}^{1-1 / 2 p}\left(J ; L_{p}\left(\Sigma, \mathbb{R}^{n+1}\right)\right) \cap L_{p}\left(J ; W_{p}^{2-1 / p}\left(\Sigma, \mathbb{R}^{n+1}\right)\right)$,

(e) $u_{0} \in W_{p}^{2-2 / p}\left(\Omega \backslash \Sigma, \mathbb{R}^{n}\right), h_{0} \in W_{p}^{3-2 / p}(\Sigma)$,

(f) $\operatorname{div} u_{0}=g_{d}(0)$ in $\Omega \backslash \Sigma$,

(g) $P_{\Sigma} \llbracket u_{0} \rrbracket+c(0, \cdot) \nabla_{\Sigma} h_{0}=P_{\Sigma} g_{u}(0)$ on $\Sigma$,

(h) $-P_{\Sigma} \llbracket \mu_{0}(\cdot)\left(\nabla u_{0}+\left[\nabla u_{0}\right]^{\mathrm{T}}\right) \rrbracket=P_{\Sigma} g(0)$ on $\Sigma$.

The solution map $\left[\left(f_{u}, g_{d}, g, P_{\Sigma} g_{u}, g_{h}, f_{h}, u_{0}, h_{0}\right) \mapsto(u, \pi, h)\right]$ is continuous between the corresponding spaces.

The proof of this result is given in the Section 7 .

3.2. Local Existence. The basic result for local well-posedness of Problem (1.1), (1.2), (1.3) in an $L_{p}$-setting is the following theorem.

Theorem 3.2. Let $p>n+2, \Omega \subset \mathbb{R}^{n}$ a bounded domain with boundary $\partial \Omega \in C^{3-}$, $\sigma, \rho_{1}, \rho_{2}>0, \rho_{1} \neq \rho_{2}$, and suppose $\psi_{j} \in C^{3}(0, \infty), \mu_{j}, d_{j} \in C^{2}(0, \infty)$ are such that

$$
\kappa_{j}(s)=-s \psi_{j}^{\prime \prime}(s)>0, \quad \mu_{j}(s)>0, \quad d_{j}(s)>0, \quad s \in(0, \infty), j=1,2 .
$$

Assume the regularity conditions

$$
\left(u_{0}, \theta_{0}\right) \in\left[W_{p}^{2-2 / p}\left(\Omega \backslash \Gamma_{0}\right)\right]^{n+1}, \quad \Gamma_{0} \in W_{p}^{3-2 / p},
$$

the compatibility conditions

$$
\begin{aligned}
& \operatorname{div} u_{0}=0 \quad \text { in } \Omega \backslash \Gamma_{0}, \quad u_{0}=\partial_{\nu} \theta_{0}=0 \quad \text { on } \partial \Omega \text {, } \\
& P_{\Gamma_{0}} \llbracket u_{0} \rrbracket=P_{\Gamma_{0}} \llbracket \mu\left(\theta_{0}\right)\left(\nabla u_{0}+\left[\nabla u_{0}\right]^{\top}\right) \nu_{\Gamma_{0}} \rrbracket=\llbracket \theta_{0} \rrbracket=0 \quad \text { on } \Gamma_{0} \text {, } \\
& l\left(\theta_{0}\right) \llbracket u_{0} \cdot \nu_{\Gamma_{0}} \rrbracket / \llbracket 1 / \rho \rrbracket+\llbracket d\left(\theta_{0}\right) \partial_{\nu_{\Gamma_{0}}} u_{0} \rrbracket=0 \quad \text { on } \Gamma_{0},
\end{aligned}
$$

and the well-posedness condition $\theta_{0}>0$ on $\bar{\Omega}$. 
Then there exists a unique $L_{p}$-solution of Problem (1.1), (1.2), (1.3) on some possibly small but nontrivial time interval $J=[0, \tau]$.

Here the notation $\Gamma_{0} \in W_{p}^{3-2 / p}$ means that $\Gamma_{0}$ is a $C^{2}$-manifold, such that its (outer) normal field $\nu_{\Gamma_{0}}$ is of class $W_{p}^{2-2 / p}\left(\Gamma_{0}\right)$. Therefore the curvature tensor $L_{\Gamma_{0}}=-\nabla_{\Gamma_{0}} \nu_{\Gamma_{0}}$ of $\Gamma_{0}$ belongs to $W_{p}^{1-2 / p}\left(\Gamma_{0}\right)$ which embeds into $C^{\alpha+1 / p}\left(\Gamma_{0}\right)$, with $\alpha=1-(n+2) / p>0$ since $p>n+2$ by assumption. For the same reason we also have $\left.u_{0} \in C^{1+\alpha}\left(\bar{\Omega}_{j}(0)\right)\right)^{n}$, and $\left.\theta_{0} \in C^{1+\alpha}\left(\bar{\Omega}_{j}(0)\right)\right), j=1,2$, and $V_{0} \in C^{1+\alpha}\left(\Gamma_{0}\right)$. The notion $L_{p}$-solution means that $(u, \pi, \theta, \Gamma)$ is obtained as the push-forward of an $L_{p}$-solution $(\bar{u}, \bar{\pi}, \bar{\theta}, h)$ of the transformed problem (2.1), which means that $(\bar{u}, \bar{\theta}, h)$ belongs to $\mathbb{E}(J)=\mathbb{E}_{u, \theta}(J) \times \mathbb{E}_{h}(J)$ defined by

$$
\mathbb{E}_{u, \theta}(J)=\left\{(u, \theta) \in\left[H_{p}^{1}\left(J ; L_{p}(\Omega)\right) \cap L_{p}\left(J ; H_{p}^{2}(\Omega \backslash \Sigma)\right)\right]^{n+1}: \operatorname{div} u=0\right\},
$$

and

$$
\mathbb{E}_{h}(J):=W_{p}^{2-1 / 2 p}\left(J ; L_{p}(\Sigma)\right) \cap H_{p}^{1}\left(J ; W_{p}^{2-1 / p}(\Sigma)\right) \cap L_{p}\left(J ; W_{p}^{3-1 / p}(\Sigma)\right) .
$$

The regularity of the pressure is obtained from the equations.

3.3. Time-Weights. For later use we need an extension of the local existence results to spaces with time weights. For this purpose, given a UMD-Banach space $Y$ and $\mu \in(1 / p, 1]$, we define for $J=\left(0, t_{0}\right)$

$$
K_{p, \mu}^{s}(J ; Y):=\left\{u \in L_{p, l o c}(J ; Y): t^{1-\mu} u \in K_{p}^{s}(J ; Y)\right\},
$$

where $s \geq 0$ and $K \in H, W$. It has been shown in [19] that the operator $d / d t$ in $L_{p, \mu}(J ; Y)$ with domain

$$
D(d / d t)={ }_{0} H_{p, \mu}^{1}(J ; Y)=\left\{u \in H_{p, \mu}^{1}(J ; Y): u(0)=0\right\}
$$

is sectorial and admits an $H^{\infty}$-calculus with angle $\pi / 2$. This is the main tool to extend Theorem 3.2 to the time weighted setting, where the solution space $\mathbb{E}(J)$ is replaced by

$$
\mathbb{E}_{\mu}(J)=\mathbb{E}_{\mu, u, \theta} \times \mathbb{E}_{\mu, h}(J),
$$

with

$$
\mathbb{E}_{\mu, u, \theta}(J)=\left\{(u, \theta) \in\left[H_{p, \mu}^{1}\left(J ; L_{p}(\Omega)\right) \cap L_{p, \mu}\left(J ; H_{p}^{2}(\Omega \backslash \Sigma)\right)\right]^{n+1}: \operatorname{div} u=0\right\},
$$

and

$$
\mathbb{E}_{\mu, h}(J):=W_{p, \mu}^{2-1 / 2 p}\left(J ; L_{p}(\Sigma)\right) \cap H_{p, \mu}^{1}\left(J ; W_{p}^{2-1 / p}(\Sigma)\right) \cap L_{p, \mu}\left(J ; W_{p}^{3-1 / p}(\Sigma)\right) .
$$

The trace spaces for $u, \theta$ and $h$ for $p>3$ are then given by

$$
\begin{aligned}
& \left(u_{0}, \theta_{0}\right) \in\left[W_{p}^{2 \mu-2 / p}(\Omega \backslash \Sigma)\right]^{n+1}, \quad h_{0} \in W_{p}^{2+\mu-2 / p}(\Sigma), \\
& h_{1}:=\partial_{t} h_{\mid t=0} \in W_{p}^{2 \mu-3 / p}(\Sigma),
\end{aligned}
$$

where for the last trace we need in addition $\mu>3 / 2 p$. Note that the embeddings

$$
\mathbb{E}_{\mu, u, \theta}(J) \hookrightarrow C\left(J ; C^{1}\left(\bar{\Omega}_{j}\right)\right)^{n+1}, \quad \mathbb{E}_{\mu, h}(J) \hookrightarrow C\left(J ; C^{2+\alpha}(\Sigma)\right) \cap C^{1}\left(J ; C^{1}(\Sigma)\right)
$$


with $\alpha=1 / 2-n / p>0$ require $\mu>1 / 2+(n+2) / 2 p$, which is feasible since $p>n+2$ by assumption. This restriction is needed for the estimation of the nonlinearities.

For these time weighted spaces we have the following result.

Corollary 3.3. Let $p>n+2, \mu \in(1 / 2+(n+2) / 2 p, 1], \sigma, \rho_{1}, \rho_{2}>0, \rho_{1} \neq \rho_{2}$, and suppose $\psi \in C^{3}(0, \infty), \mu, d \in C^{2}(0, \infty)$ are such that

$$
\kappa_{j}(s)=-s \psi_{j}^{\prime \prime}(s)>0, \quad \mu_{j}(s)>0, \quad d_{j}(s)>0, \quad s \in(0, \infty), j=1,2 .
$$

Assume the regularity conditions

$$
\left(u_{0}, \theta_{0}\right) \in\left[W_{p}^{2 \mu-2 / p}\left(\Omega \backslash \Gamma_{0}\right)\right]^{n+1}, \quad \Gamma_{0} \in W_{p}^{2+\mu-2 / p},
$$

\section{the compatibility conditions}

$$
\begin{aligned}
& \operatorname{div} u_{0}=0 \quad \text { in } \Omega \backslash \Sigma, \quad u_{0}=\partial_{\nu} \theta_{0}=0 \quad \text { on } \partial \Omega, \\
& P_{\Gamma_{0}} \llbracket u_{0} \rrbracket=P_{\Gamma_{0}} \llbracket \mu\left(\theta_{0}\right)\left(\nabla u_{0}+\left[\nabla u_{0}\right]^{\top}\right) \nu_{\Gamma_{0}} \rrbracket=\llbracket \theta_{0} \rrbracket=0 \\
& l\left(\theta_{0}\right) \llbracket u_{0} \cdot \nu_{\Gamma_{0}} \rrbracket / \llbracket 1 / \rho \rrbracket+\llbracket d\left(\theta_{0}\right) \partial_{\nu_{\Gamma_{0}}} u_{0} \rrbracket=0 \quad \text { on } \Gamma_{0},
\end{aligned}
$$

as well as the well-posedness condition $\theta_{0}>0$ on $\bar{\Omega}$.

Then the transformed problem (2.1) admits a unique solution $z=(u, \theta, h) \in$ $\mathbb{E}_{\mu}(0, \tau)$ for some nontrivial time interval $J=[0, \tau]$. The solution depends continuously on the data. For each $\delta>0$ the solution belongs to $\mathbb{E}(\delta, \tau)$, i.e. regularizes instantly.

3.4. Proofs. The proof of Theorem 3.2 follows similar ideas as in the papers 14 for the two-phase Navier-Stokes problem without phase transition, 22] for the problem with phase transitions and equal densities and 23] for the case of nonequal densities and nearly flat interface. Therefore we refrain from presenting all details but instead concentrate on the main ideas; note that the spaces are the same as in [23].

The transformed problem is rewritten in quasilinear form, dropping the bars and collecting its principal linear part on the left hand side. We set $\mu_{0}(x)=$ $\mu\left(\theta_{0}(x)\right), \kappa_{0}(x)=\kappa\left(\theta_{0}(x)\right), d_{0}(x)=d\left(\theta_{0}(x)\right), c(t, x)=-e^{\Delta_{\Sigma} t} \llbracket u_{0} \cdot \nu_{\Sigma} \rrbracket$, and $b(t, x)=e^{\Delta_{\Sigma} t} \llbracket \rho u_{0} \rrbracket$. Then, eliminating $j$, the problems reads as follows

$$
\begin{aligned}
& \rho \partial_{t} u-\mu_{0}(x) \Delta u+\nabla \pi=F_{u}(u, \pi, \theta, h) \quad \text { in } \quad \Omega \backslash \Sigma, \\
& \operatorname{div} u=G_{d}(u, h) \quad \text { in } \quad \Omega \backslash \Sigma, \\
& \rho \kappa_{0}(x) \partial_{t} \theta-d_{0}(x) \Delta \theta=F_{\theta}(u, \theta, h) \quad \text { on } \quad \Omega \backslash \Sigma \text {, } \\
& u=\partial_{\nu} \theta=0 \quad \text { on } \partial \Omega \text {, } \\
& u(0)=u_{0}, \quad \theta(0)=\theta_{0} \quad \text { in } \Omega \backslash \Sigma,
\end{aligned}
$$




$$
\begin{aligned}
& P_{\Sigma} \llbracket u \rrbracket+c(t, x) \nabla_{\Sigma} h=G_{j}(u, \theta, h) \quad \text { on } \quad \Sigma, \\
& -P_{\Sigma} \llbracket \mu_{0}(x)\left(\nabla u+[\nabla u]^{\top}\right) \nu_{\Sigma} \rrbracket=G_{u}^{\tan }(u, \theta, h) \quad \text { on } \quad \Sigma \text {, } \\
& -2 \llbracket \mu_{0}(x) \nabla u \rrbracket \nu_{\Sigma} \cdot \nu_{\Sigma}+\llbracket \pi \rrbracket-\sigma \Delta_{\Sigma} h=G_{u}^{n o r}(u, \theta, h) \quad \text { on } \quad \Sigma \text {, } \\
& \llbracket \theta \rrbracket=0 \quad \text { on } \Sigma \text {, } \\
& -\llbracket d_{0}(x) \partial_{\nu_{\Sigma}} \theta \rrbracket=G_{\theta}(\theta, h) \quad \text { on } \quad \Sigma, \\
& -2 \llbracket\left(\mu_{0}(x) / \rho\right) \nabla u \rrbracket \nu_{\Sigma} \cdot \nu_{\Sigma}+\llbracket \pi / \rho \rrbracket=G_{h}(u, \theta, h) \quad \text { on } \quad \Sigma \text {, } \\
& \llbracket \rho \rrbracket \partial_{t} h-\llbracket \rho u \cdot \nu_{\Sigma} \rrbracket+b(t, x) \cdot \nabla_{\Sigma} h=F_{h}(u, h) \quad \text { on } \quad \Sigma \text {, } \\
& h(0)=h_{0} \quad \text { on } \Sigma \text {. }
\end{aligned}
$$

The nonlinearities are given by

$$
\begin{aligned}
& F_{u}(u, \theta, \pi, h)=\left(\mu(\theta)-\mu\left(\theta_{0}\right)\right) \Delta u+M_{1}(h) \nabla \pi-\rho\left(u \cdot\left(I-M_{1}(h)\right)-R(h) \cdot\right) \nabla u \\
& +\mu^{\prime}(\theta)\left(\left(I-M_{1}(h)\right) \nabla \theta \cdot\left(\left(I-M_{1}(h)\right) \nabla u+\left[\left(I-M_{1}(h)\right) \nabla u\right]^{\top}\right)\right) \\
& -\mu(\theta)\left(M_{2}(h): \nabla^{2}\right) u-\mu(\theta)\left(M_{3}(h) \cdot \nabla\right) u+\mu(\theta) M_{4}(h): \nabla u, \\
& G_{d}(u, h)=M_{1}(h): \nabla u, \\
& F_{\theta}(u, \theta, h)=\rho\left(\kappa\left(\theta_{0}\right)-\kappa(\theta)\right) \partial_{t} \theta-\left(d(\theta)-d\left(\theta_{0}\right)\right) \Delta \theta-d(\theta) M_{2}(h): \nabla^{2} \theta \\
& +d^{\prime}(\theta)\left|\left(I-M_{1}(h)\right) \nabla \theta\right|^{2}-d(\theta) M_{3}(h) \cdot \nabla \theta-\rho \kappa(\theta)(R(h) \cdot \nabla) \theta \\
& -\rho \kappa(\theta) u \cdot\left(I-M_{1}(h)\right) \nabla \theta \\
& +\mu(\theta)\left(\left(I-M_{1}(h)\right) \nabla u+\left[\left(I-M_{1}(h)\right) \nabla u\right]^{T}\right):\left(I-M_{1}(h)\right) \nabla u, \\
& G_{j}(u, h)=\llbracket u \cdot \nu_{\Sigma} \rrbracket M_{0}(h)-e^{\Delta_{\Sigma} t} \llbracket u_{0} \cdot \nu_{\Sigma} \rrbracket \nabla_{\Sigma} h, \\
& G_{u}^{\text {tan }}(u, \theta, h)=P_{\Sigma} \llbracket\left(\mu(\theta)-\mu\left(\theta_{0}\right)\right)\left(\nabla u+[\nabla u]^{T}\right) \nu_{\Sigma} \rrbracket \\
& -P_{\Sigma} \llbracket \mu(\theta)\left(\nabla u+\left[\nabla u \rrbracket^{\top}\right) M_{0}(h) \nabla_{\Sigma} h \rrbracket\right. \\
& -P_{\Sigma} \llbracket \mu(\theta)\left(M_{1}(h) \nabla u+\left[M_{1}(h) \nabla u \rrbracket^{\top}\right)\left(\nu_{\Sigma}-M_{0}(h) \nabla_{\Sigma} h\right) \rrbracket\right. \\
& +\llbracket \mu(\theta)\left(\left(I-M_{1}\right) \nabla u+\left[\left(I-M_{1}\right) \nabla u\right]^{T}\right) \\
& \left(\nu_{\Sigma}-M_{0} \nabla_{\Sigma} h\right) \cdot \nu_{\Sigma} \rrbracket M_{0}(h) \nabla_{\Sigma} h, \\
& G_{u}^{n o r}(u, \theta, h)=\llbracket\left(\mu(\theta)-\mu\left(\theta_{0}\right)\right)\left(\nabla u+[\nabla u]^{T}\right) \nu_{\Sigma} \cdot \nu_{\Sigma} \rrbracket \\
& -\llbracket \mu(\theta)\left(\nabla u+[\nabla u]^{\top}\right) M_{0}(h) \nabla_{\Sigma} h \cdot \nu_{\Sigma} \rrbracket \\
& -\llbracket \mu(\theta)\left(M_{1}(h) \nabla u+\left[M_{1}(h) \nabla u\right]^{\top}\right)\left(\nu_{\Sigma}-M_{0}(h) \nabla_{\Sigma} h\right) \cdot \nu_{\Sigma} \rrbracket \\
& +\sigma\left(H_{\Gamma}(h)-\Delta_{\Sigma} h\right)-\llbracket u \cdot \nu_{\Gamma} \rrbracket / \llbracket 1 / \rho \rrbracket, \\
& G_{\theta}(\theta, h)=l(\theta) j-\llbracket d_{0}(x) \partial_{\nu} \theta \rrbracket+\llbracket d(\theta)\left(I-M_{1}(h)\right) \nabla \theta \cdot \nu_{\Gamma} \rrbracket, \\
& G_{h}(u, \theta, h)=-\llbracket \psi(\theta) \rrbracket-\llbracket 1 / 2 \rho^{2} \rrbracket j^{2} \\
& +2 \llbracket(\mu(x) / \rho) \partial_{\nu} u \rrbracket-\llbracket(\mu(\theta) / \rho)\left(M_{1}(h) \nabla u+\left[M_{1}(h) \nabla u\right]^{\top}\right) \nu_{\Gamma} \cdot \nu_{\Gamma} \rrbracket, \\
& F_{h}(u, h)=\left(b(t, x)-\llbracket \rho M_{0}(h) u \rrbracket\right) \nabla_{\Sigma} h, \\
& j=\llbracket u \cdot \nu_{\Sigma} \rrbracket / \beta(h) \llbracket 1 / \rho \rrbracket, \quad \nu_{\Gamma}=\beta(h)\left(\nu_{\Sigma}-M_{0}(h) \nabla_{\Sigma} h\right) .
\end{aligned}
$$


Here we employed the abbreviations

$$
\begin{aligned}
& M_{1}(h)=\left[D \xi_{h}\right]^{\top}\left[I+D \xi_{h}\right]^{-\mathrm{\top}} \nabla \bar{\pi}, \\
& M_{2}(h)=M_{1}(h)+M_{1}^{\top}(h)-M_{1}(h) M_{1}^{\top}(h), \\
& M_{3}(h)=\left(I-M_{1}(h)\right) \operatorname{div} M_{2}(h), \\
& M_{4}(h)=\left(\left(I-M_{1}(h)\right) \nabla\right) M_{1}(h)-\left[\left(\left(I-M_{1}(h)\right) \nabla\right) M_{1}(h)\right]^{\top} .
\end{aligned}
$$

We prove local well-posedness of (3.7), (3.8) by means of maximal $L_{p}$-regularity of the linear problem (Theorem 3.1) and the contraction mapping principle. The right hand side of problem (3.7), (3.8) consist of either lower order terms, or terms of the same order as those appearing on the left hand side but carry factors which can be made small by construction. Indeed, we have smallness of $h_{0}, \nabla_{\Sigma} h_{0}$ and even of $\nabla_{\Sigma}^{2} h_{0}$ uniformly on $\Sigma$, because $\Gamma_{0}$ is approximated by $\Sigma$ in the second order bundle. $\theta$ appears nonlinearly in $\psi, \kappa, \mu, d$, but only to order zero; hence e.g. the difference $\left(\mu(\theta(t))-\mu\left(\theta_{0}\right)\right)$ will be uniformly small for small times.

We introduce appropriate function spaces. Let $J=[0, a]$. The solution spaces are defined by

$$
\begin{aligned}
& \mathbb{E}_{1}(a):=\left\{u \in H_{p}^{1}\left(J ; L_{p}(\Omega)\right)^{n} \cap L_{p}\left(J ; H_{p}^{2}(\Omega \backslash \Sigma)\right)^{n}: u=0 \text { on } \partial \Omega,\right\}, \\
& \mathbb{E}_{2}(a):=L_{p}\left(J ; \dot{H}_{p}^{1}(\Omega \backslash \Sigma)\right), \\
& \mathbb{E}_{3}(a):=\left[W_{p}^{1 / 2-1 / 2 p}\left(J ; L_{p}(\Sigma)\right) \cap L_{p}\left(J ; W_{p}^{1-1 / p}(\Sigma)\right)\right]^{2}, \\
& \mathbb{E}_{4}(a):=\left\{\theta \in H_{p}^{1}\left(J ; L_{p}(\Omega)\right) \cap L_{p}\left(J ; H_{p}^{2}(\Omega \backslash \Sigma)\right): \partial_{\nu} \theta=0 \text { on } \partial \Omega, \llbracket \theta \rrbracket=0\right\}, \\
& \mathbb{E}_{5}(a):=W_{p}^{2-1 / 2 p}\left(J ; L_{p}(\Sigma)\right) \cap H_{p}^{1}\left(J ; W_{p}^{2-1 / p}(\Sigma)\right) \cap L_{p}\left(J ; W_{p}^{3-1 / p}(\Sigma)\right) .
\end{aligned}
$$

We abbreviate

$$
\mathbb{E}(a):=\left\{\left(u, \pi, \pi_{j}, \theta, h\right) \in \mathbb{E}_{1}(a) \times \mathbb{E}_{2}(a) \times \mathbb{E}_{3}(a) \times \mathbb{E}_{4}(a) \times \mathbb{E}_{5}(a)\right\},
$$

and equip $\mathbb{E}_{j}(a)(j=1, \ldots, 5)$ with their natural norms, which turns $\mathbb{E}(a)$ into a Banach space. A left subscript 0 always means that the time trace at $t=0$ of the function in question is zero whenever it exists.

The data spaces are defined by

$$
\begin{aligned}
& \mathbb{F}_{1}(a):=L_{p}\left(J ; L_{p}(\Omega)\right)^{n}, \\
& \mathbb{F}_{2}(a):=H_{p}^{1}\left(J ; \dot{H}_{p}^{-1}(\Omega)\right) \cap L_{p}\left(J ; H_{p}^{1}(\Omega \backslash \Sigma)\right), \\
& \mathbb{F}_{3}(a):=P_{\Sigma}\left[W_{p}^{1-1 / 2 p}\left(J ; L_{p}\left(\Sigma ; \mathbb{R}^{n}\right)\right) \cap L_{p}\left(J ; W_{p}^{2-1 / p}\left(\Sigma, \mathbb{R}^{n}\right)\right)\right] \\
& \mathbb{F}_{4}(a):=W_{p}^{1 / 2-1 / 2 p}\left(J ; L_{p}(\Sigma)\right)^{n} \cap L_{p}\left(J ; W_{p}^{1-1 / p}(\Sigma)\right)^{n}, \\
& \mathbb{F}_{5}(a):=L_{p}\left(J ; L_{p}(\Omega)\right), \\
& \mathbb{F}_{6}(a):=W_{p}^{1 / 2-1 / 2 p}\left(J ; L_{p}(\Sigma)\right) \cap L_{p}\left(J ; W_{p}^{1-1 / p}(\Sigma)\right), \\
& \mathbb{F}_{7}(a):=W_{p}^{1 / 2-1 / 2 p}\left(J ; L_{p}(\Sigma)\right) \cap L_{p}\left(J ; W_{p}^{1-1 / p}(\Sigma)\right), \\
& \mathbb{F}_{8}(a):=W_{p}^{1-1 / 2 p}\left(J ; L_{p}(\Sigma)\right) \cap L_{p}\left(J ; W_{p}^{2-1 / p}(\Sigma)\right) .
\end{aligned}
$$


We abbreviate

$$
\mathbb{F}(a):=\left\{\left(f_{u}, g_{d}, g_{j}, g_{u}, f_{\theta}, g_{\theta}, g_{h}, f_{h}\right) \in \prod_{k=1}^{8} \mathbb{F}_{k}(a)\right\},
$$

and equip $\mathbb{F}_{k}(a)(k=1, \ldots, 8)$ with their natural norms, which turns $\mathbb{F}(a)$ into a Banach space.

Step 1. In order to economize our notation, we set $z=\left(u, \pi, \pi_{1}, \pi_{2}, \theta, h\right) \in \mathbb{E}(a)$ and reformulate the quasilinear problem (3.7), (3.8) as

$$
L z=N(z) \quad(u(0), \theta(0), h(0))=\left(u_{0}, \theta_{0}, h_{0}\right),
$$

where $L$ denotes the linear operator on the left hand side of (3.7), (3.8), and $N$ denotes the nonlinear mapping on the right-hand side of (3.7), (3.8). From Theorem 3.1 we know that $L: \mathbb{E}(a) \rightarrow \mathbb{F}(a)$ is bounded and linear, and that $L:{ }_{0} \mathbb{E}(a) \rightarrow{ }_{0} \mathbb{F}(a)$ is an isomorphism for each $a>0$, with norm independent of $0<a \leq a_{0}<\infty$.

Concerning the nonlinearity $N$, we have the following result.

Proposition 3.4. Suppose $p>n+2, \sigma>0$, and let $\psi_{i} \in C^{3}(0, \infty), \mu_{i}, d_{i} \in$ $C^{2}(0, \infty), \kappa_{i}(s)=-s \psi^{\prime \prime}(s)$, for $i=1,2$.

Then for each $a>0$ the nonlinearity satisfies $N \in C^{1}(\mathbb{E}(a), \mathbb{F}(a))$ and its Fréchet derivative $N^{\prime}$ satisfies in addition $N^{\prime}\left(u, \pi, \pi_{1}, \pi_{2}, \theta, h\right) \in \mathcal{B}\left({ }_{0} \mathbb{E}(a),{ }_{0} \mathbb{F}(a)\right)$. Moreover, there is $\eta>0$ such that for a given $z_{*} \in \mathbb{E}\left(a_{0}\right)$ with $\left|h_{0}\right|_{C^{2}(\Sigma)} \leq \eta$, there are continuous functions $\alpha(r)>0$ and $\beta(a)>0$ with $\alpha(0)=\beta(0)=0$, such that

$$
\left\|N^{\prime}\left(\bar{z}+z_{*}\right)\right\|_{\mathcal{B}\left({ }_{0} \mathbb{E}(a),{ }_{0} \mathbb{F}(a)\right)} \leq \alpha(r)+\beta(a), \quad \bar{z} \in \mathbb{B}_{r} \subset{ }_{0} \mathbb{E}(a) .
$$

This proposition is proved estimating the nonlinearities in the same way as in 22 and 23 .

Step 2. We reduce the problem to initial values 0 and resolve the compatibilities as follows. Thanks to Proposition 4.1 in [14], we find extensions $g_{d}^{*} \in \mathbb{F}_{2}(a)$, $g_{u}^{*} \in \mathbb{F}_{3}(a)$ which satisfy

$$
g_{d}^{*}(0)=\operatorname{div} u_{0}, \quad P_{\Sigma} g_{u}^{*}(0)=-P_{\Sigma} \llbracket \mu_{0}\left(\nabla u_{0}+\left[\nabla u_{0}\right]^{\top}\right) \nu_{\Sigma} \rrbracket .
$$

Further we define

$$
g_{j}^{*}:=e^{\Delta_{\Sigma} t} G_{j}\left(\theta_{0}, h_{0}\right), \quad g_{\theta}^{*}:=e^{\Delta_{\Sigma} t} G_{\theta}\left(u_{0}, \theta_{0}, h_{0}\right),
$$

and set $f_{u}^{*}=f_{\theta}^{*}=f_{h}^{*}=g_{h}^{*}=0$. With these extensions, by Theorem 3.1 we may solve the linear problem (3.1), (3.2), (3.3) with initial data $\left(u_{0}, \theta_{0}, h_{0}\right)$ and inhomogeneities $\left(f_{u}^{*}, g_{d}^{*}, g_{j}^{*}, g_{u}^{*}, f_{\theta}^{*}, g_{\theta}^{*}, g_{h}^{*}, f_{h}^{*}\right)$, which satisfy the required regularity conditions and, by construction, the compatibility conditions, to obtain a unique solution

$$
z^{*}=\left(u^{*}, \pi^{*}, \pi_{1}^{*}, \pi_{2}^{*}, \theta^{*}, h^{*}\right) \in \mathbb{E}(a)
$$

with $u^{*}(0)=u_{0}, \theta^{*}(0)=\theta_{0}$, and $h^{*}(0)=h_{0}$.

Step 3. We rewrite problem (3.9) as

$$
L z=N\left(z+z^{*}\right)-L z^{*}=: K(z), \quad z \in_{0} \mathbb{E}(a) .
$$


The solution is given by the fixed point problem $z=L^{-1} K(z)$, since Theorem 3.1 implies that $L:{ }_{0} \mathbb{E}(a) \rightarrow{ }_{0} \mathbb{F}(a)$ is an isomorphism with

$$
\left|L^{-1}\right|_{\mathcal{L}\left({ }_{0} \mathbb{F}(a),{ }_{0} \mathbb{E}(a)\right)} \leq M, \quad a \in\left(0, a_{0}\right],
$$

where $M$ is independent of $a \leq a_{0}$. We may assume that $M \geq 1$. Thanks to Proposition 3.4 and due to $K(0)=N\left(z^{*}\right)-L z^{*}$, we may choose $a \in\left(0, a_{0}\right]$ and $r>0$ sufficiently small such that

$$
|K(0)|_{\mathbb{F}(a)} \leq \frac{r}{2 M}, \quad\left|K^{\prime}(z)\right|_{\mathcal{L}\left({ }_{0} \mathbb{E}(a),{ }_{0} \mathbb{F}(a)\right)} \leq \frac{1}{2 M}, \quad z \in{ }_{0} \mathbb{E}(a), \quad|z|_{\mathbb{E}(a)} \leq r
$$

hence

$$
|K(z)|_{\mathbb{F}(a)} \leq \frac{r}{M},
$$

which ensures that $L^{-1} K(z): \mathbb{B}_{0} \mathbb{E}(a)(0, r) \rightarrow \mathbb{B}_{0} \mathbb{E}(a)(0, r)$ is a contraction. Thus we may employ the contraction mapping principle to obtain a unique solution on a time interval $[0, a]$, which completes the proof of Theorem 3.2. Corollary 3.3 is obtained in the same way.

\section{Linear Stability of Equilibria}

1. We call an equilibrium non-degenerate if the balls do neither touch each other nor the outer boundary; this set is denoted by $\mathcal{E}$. To derive the full linearization at a non-degenerate equilibrium $e_{*}:=\left(0, \theta_{*}, \Sigma\right) \in \mathcal{E}$, note that the quadratic terms $u \cdot \nabla u, u \cdot \nabla \theta,|D(u)|_{2}^{2}, \llbracket u \rrbracket j$, and $\llbracket 1 / 2 \rho^{2} \rrbracket j^{2}$ give no contribution to the linearization. Therefore we obtain the following fully linearized problem.

$$
\begin{aligned}
\rho \partial_{t} u-\mu_{*} \Delta u+\nabla \pi=\rho f_{u} & \text { in } \Omega \backslash \Sigma, \\
\operatorname{div} u=g_{d} & \text { in } \Omega \backslash \Sigma, \\
P_{\Sigma} \llbracket u \rrbracket=P_{\Sigma} g_{u} & \text { on } \Sigma, \\
-\llbracket T(u, \pi, \vartheta) \nu_{\Sigma} \rrbracket+\sigma \mathcal{A}_{\Sigma} h \nu_{\Sigma}=g & \text { on } \Sigma, \\
u=0 & \text { on } \partial \Omega, \\
u=u_{0} & \text { in } \Omega,
\end{aligned}
$$

where $\mu_{*}=\mu\left(\theta_{*}\right)$ and $\mathcal{A}_{\Sigma}=-H^{\prime}(0)=-(n-1) / R_{*}^{2}-\Delta_{\Sigma}$. For the relative temperature $\vartheta=\left(\theta-\theta_{*}\right) / \theta_{*}$ we obtain

$$
\begin{aligned}
\rho \kappa_{*} \partial_{t} \vartheta-d_{*} \Delta \vartheta=\rho \kappa_{*} f_{\theta} & \text { in } \Omega \backslash \Sigma, \\
\llbracket \vartheta \rrbracket=0 & \text { on } \Sigma, \\
-\left(l_{*} / \theta_{*}\right) \llbracket u \cdot \nu_{\Sigma} \rrbracket / \llbracket 1 / \rho \rrbracket-\llbracket d_{*} \partial_{\nu_{\Sigma}} \vartheta \rrbracket=g_{\theta} & \text { on } \Sigma, \\
\partial_{\nu} \vartheta=0 & \text { on } \partial \Omega, \\
\vartheta=\vartheta_{0} & \text { in } \Omega,
\end{aligned}
$$


with $\kappa_{*}=\kappa\left(\theta_{*}\right), d_{*}=d\left(\theta_{*}\right)$ and $l_{*}=l\left(\theta_{*}\right)$. The remaining conditions on the equilibrium interface $\Sigma$ are

$$
\begin{aligned}
\llbracket-\rho^{-1} T(u, \pi, \vartheta) \nu_{\Sigma} \cdot \nu_{\Sigma} \rrbracket+l_{*} \vartheta & =g_{h} & & \text { on } \Sigma, \\
\partial_{t} h-\llbracket \rho u \cdot \nu_{\Sigma} \rrbracket / \llbracket \rho \rrbracket & =f_{h} & & \text { on } \Sigma, \\
h(0) & =h_{0} & & \text { on } \Sigma .
\end{aligned}
$$

The time-trace space $\mathbb{E}_{\gamma}$ of $\mathbb{E}(J)$ is given by

$$
\left(u_{0}, \vartheta_{0}, h_{0}\right) \in \mathbb{E}_{\gamma}=\left[W_{p}^{2-2 / p}((\Omega \backslash \Sigma)]^{n+1} \times W_{p}^{3-2 / p}(\Sigma),\right.
$$

and the space of data is

$$
\begin{aligned}
\left(\left(f_{u}, f_{\theta}\right), g_{d},\left(f_{h}, P_{\Sigma} g_{u}\right),\left(g, g_{\theta}, g_{h}\right)\right) & \in \mathbb{F}(J) \\
& :=\mathbb{F}_{u, \theta}(J) \times \mathbb{F}_{d}(J) \times \mathbb{F}_{h}(J)^{n+1} \times \mathbb{F}_{\theta}(J)^{n+2},
\end{aligned}
$$

where

$$
\mathbb{F}_{u, \theta}(J)=L_{p}(J \times \Omega)^{n+1}, \quad \mathbb{F}_{d}(J)=H_{p}^{1}\left(J ; \dot{H}_{p}^{-1}(\Omega)\right) \cap L_{p}\left(J ; H_{p}^{1}(\Omega)\right),
$$

and

$$
\begin{gathered}
\mathbb{F}_{\theta}(J)=W_{p}^{1 / 2-1 / 2 p}\left(J ; L_{p}(\Sigma)\right) \cap L_{p}\left(J ; W_{p}^{1-1 / p}(\Sigma)\right), \\
\mathbb{F}_{h}(J)=W_{p}^{1-1 / 2 p}\left(J ; L_{p}(\Sigma)\right) \cap L_{p}\left(J ; W_{p}^{2-1 / p}(\Sigma)\right) .
\end{gathered}
$$

Then by localization and coordinate transformations it follows from the maximal regularity result in 23 that the operator $\mathbb{L}$ defined by the left hand side of (4.1), (4.2), (4.3) is an isomorphism from $\mathbb{E}$ into $\mathbb{F} \times \mathbb{E}_{\gamma}$; see also Section 7. The range of $\mathbb{L}$ is determined by the natural compatibility conditions. If the time derivatives $\partial_{t}$ are replaced by $\partial_{t}+\omega, \omega>0$ sufficiently large, then this result is also true for $J=\mathbb{R}_{+}$.

2. We introduce a functional analytic setting as follows. Set

$$
X_{0}=L_{p, \sigma}(\Omega) \times L_{p}(\Omega) \times W_{p}^{2-1 / p}(\Sigma),
$$

where the subscript $\sigma$ means solenoidal, and define the operator $L$ by

$$
L(u, \vartheta, h)=\left(-\left(\mu_{*} / \rho\right) \Delta u+\nabla \pi / \rho,-\left(d_{*} / \rho \kappa_{*}\right) \Delta \vartheta,-\llbracket \rho u \cdot \nu_{\Sigma} \rrbracket / \llbracket \rho \rrbracket\right) .
$$

To define the domain $D(L)$ of $L$, we set

$$
\begin{array}{r}
X_{1}=\left\{(u, \vartheta, h) \in H_{p}^{2}(\Omega \backslash \Sigma)^{n+1} \times W_{p}^{3-1 / p}(\Sigma): \operatorname{div} u=0 \text { in } \Omega \backslash \Sigma,\right. \\
\left.P_{\Sigma} \llbracket u \rrbracket=\llbracket \vartheta \rrbracket=0 \text { on } \Sigma, u=\partial_{\nu} \vartheta=0 \text { on } \partial \Omega\right\},
\end{array}
$$

and

$$
D(L)=\left\{(u, \vartheta, h) \in X_{1}: P_{\Sigma} \llbracket \mu_{*} D(u) \nu_{\Sigma} \rrbracket=0, \quad\left(l_{*} / \theta_{*}\right) j+\llbracket d_{*} \partial_{\nu_{\Sigma}} \vartheta \rrbracket=0 \quad \text { on } \Sigma\right\} .
$$


Here $j$ is given by $j=\llbracket u \cdot \nu_{\Sigma} \rrbracket / \llbracket \rho^{-1} \rrbracket$, and $\pi$ is determined as the solution of the weak transmission problem

$$
\begin{aligned}
& (\nabla \pi \mid \nabla \phi / \rho)_{2}=\left(\left(\mu_{*} / \rho\right) \Delta u \mid \nabla \phi\right)_{2}, \quad \phi \in \dot{H}_{p^{\prime}}^{1}(\Omega), \\
& \llbracket \pi \rrbracket=-\sigma \mathcal{A}_{\Sigma} h+2 \llbracket \mu_{*}\left(D(u) \nu_{\Sigma} \mid \nu_{\Sigma}\right) \rrbracket, \quad \text { on } \Sigma, \\
& \llbracket \pi / \rho \rrbracket=2 \llbracket\left(\mu_{*} / \rho\right)\left(D(u) \nu_{\Sigma} \mid \nu_{\Sigma}\right) \rrbracket-l_{*} \vartheta \quad \text { on } \Sigma .
\end{aligned}
$$

Let us introduce solution operators $T_{j}, j \in\{1,2,3\}$ as follows

$$
\begin{aligned}
\frac{1}{\rho} \nabla \pi & =T_{1}\left(\left(\mu_{*} / \rho\right) \Delta u\right)+T_{2}\left(-\sigma \mathcal{A}_{\Sigma} h+2 \llbracket \mu_{*}\left(D(u) \nu_{\Sigma} \mid \nu_{\Sigma}\right) \rrbracket\right) \\
& +T_{3}\left(2 \llbracket\left(\mu_{*} / \rho\right)\left(D(u) \nu_{\Sigma} \mid \nu_{\Sigma}\right) \rrbracket-l_{*} \vartheta\right) .
\end{aligned}
$$

We refer to Köhne, Prüss and Wilke [14 for the analysis of such transmission problems.

Then the linearized problem can be rewritten as an abstract evolution problem in $X_{0}$.

$$
\dot{z}+L z=f, \quad t>0, \quad z(0)=z_{0},
$$

where $z=(u, \vartheta, h), f=\left(f_{u}, f_{\theta}, f_{h}\right), z_{0}=\left(u_{0}, \vartheta_{0}, h_{0}\right)$, provided $g_{d}=g_{u}=g=$ $g_{\theta}=g_{h}=0$. The linearized problem has maximal $L_{p}$-regularity, hence (4.4) has this property as well. Therefore, by a result due to Hieber and Prüss, $-L$ generates an analytic $C_{0}$-semigroup in $X_{0}$; cf. Prüss [18, Proposition 1.1.

Since the embedding $X_{1} \hookrightarrow X_{0}$ is compact, the semigroup $e^{-L t}$ as well as the resolvent $(\lambda+L)^{-1}$ of $-L$ are compact, too. Therefore the spectrum $\sigma(L)$ of $L$ consists of countably many eigenvalues of finite algebraic multiplicity, and it is independent of $p$.

3. We concentrate now on the case $l_{*} \neq 0$. Suppose that $\lambda$ with $\operatorname{Re} \lambda \geq 0$ is an eigenvalue of $-L$. This means

$$
\begin{aligned}
\lambda \rho u-\mu_{*} \Delta u+\nabla \pi & =0 & & \text { in } \Omega \backslash \Sigma, \\
\operatorname{div} u & =0 & & \text { in } \Omega \backslash \Sigma, \\
P_{\Sigma} \llbracket u \rrbracket & =0 & & \text { on } \Sigma, \\
-\llbracket \mu_{*} D(u) \nu_{\Sigma} \rrbracket+\llbracket \pi \rrbracket+\sigma \mathcal{A}_{\Sigma} h \nu_{\Sigma} & =0 & & \text { on } \Sigma, \\
u & =0 & & \text { on } \partial \Omega, \\
\lambda \rho \kappa_{*} \vartheta-d_{*} \Delta \vartheta & =0 & & \text { in } \Omega \backslash \Sigma, \\
\llbracket \vartheta \rrbracket & =0 & & \text { on } \Sigma, \\
-\left(l_{*} / \theta_{*}\right) \llbracket u \cdot \nu_{\Sigma} \rrbracket / \llbracket 1 / \rho \rrbracket-\llbracket d_{*} \partial_{\nu_{\Sigma}} \vartheta \rrbracket & =0 & & \text { on } \Sigma, \\
\partial_{\nu} \vartheta & =0 & & \text { on } \partial \Omega, \\
& & & \\
\llbracket-\rho^{-1} T(u, \pi, \vartheta) \nu_{\Sigma} \cdot \nu_{\Sigma} \rrbracket+l_{*} \vartheta & =0 & & \text { on } \Sigma, \\
\lambda \llbracket \rho \rrbracket h-\llbracket \rho u \cdot \nu_{\Sigma} \rrbracket & =0 & & \text { on } \Sigma .
\end{aligned}
$$


Observe that on $\Sigma$ we may write

$$
u_{k}=P_{\Sigma} u+\lambda h \nu_{\Sigma}+j \nu_{\Sigma} / \rho_{k}, \quad k=1,2 .
$$

By this identity, taking the inner product of the problem for $u$ with $u$ and integrating by parts we get

$$
\begin{aligned}
0=\lambda\left|\rho^{1 / 2} u\right|_{2}^{2} & -(\operatorname{div} T(u, \pi, \vartheta) \mid u)_{2} \\
=\lambda\left|\rho^{1 / 2} u\right|_{2}^{2} & +\int_{\Omega} T(u, \pi, \vartheta): \nabla \bar{u} d x \\
& +\int_{\Sigma}\left(T_{2}\left(u_{2}, \pi_{2}, \vartheta \vartheta_{2}\right) \nu_{\Sigma} \cdot \bar{u}_{2}-T_{1}\left(u_{1}, \pi_{1}, \vartheta_{1}\right) \nu_{\Sigma} \cdot \bar{u}_{1}\right) d \Sigma \\
=\lambda\left|\rho^{1 / 2} u\right|_{2}^{2} & +2\left|\mu_{*}^{1 / 2} D(u)\right|_{2}^{2} \\
& +\left(\llbracket T(u, \pi, \vartheta) \nu_{\Sigma} \rrbracket \mid P_{\Sigma} u+\lambda h \nu_{\Sigma}\right)_{\Sigma}+\left(\llbracket T(u, \pi, \vartheta) \nu_{\Sigma} \cdot \nu_{\Sigma} / \rho \rrbracket \mid j\right)_{\Sigma} \\
=\lambda\left|\rho^{1 / 2} u\right|_{2}^{2} & +2\left|\mu_{*}^{1 / 2} D(u)\right|_{2}^{2}+\sigma \bar{\lambda}\left(\mathcal{A}_{\Sigma} h \mid h\right)_{\Sigma}+l_{*}(\vartheta \mid j)_{\Sigma},
\end{aligned}
$$

since $\llbracket T(u, \pi, \vartheta) \nu_{\Sigma} \rrbracket=\sigma \mathcal{A}_{\Sigma} h \nu_{\Sigma}$ and $\llbracket T(u, \pi, \vartheta) \nu_{\Sigma} \cdot \nu_{\Sigma} / \rho \rrbracket=l_{*} \vartheta$. On the other hand, the inner product of the equation for $\vartheta$ with $\vartheta$ by an integration by parts and $\llbracket \vartheta \rrbracket=0$ leads to

$$
\begin{aligned}
0 & =\lambda\left|\left(\rho \kappa_{*}\right)^{1 / 2} \vartheta\right|_{2}^{2}+\left|d_{*}^{1 / 2} \nabla \vartheta\right|_{2}^{2}+\left(\llbracket d_{*} \partial_{\nu_{\Sigma}} \vartheta \rrbracket \mid \vartheta\right)_{\Sigma} \\
& =\lambda\left|\left(\rho \kappa_{*}\right)^{1 / 2} \vartheta\right|_{2}^{2}+\left|d_{*}^{1 / 2} \nabla \vartheta\right|_{2}^{2}-l_{*}(j \mid \vartheta)_{\Sigma} / \theta_{*},
\end{aligned}
$$

where we employed $\left(l_{*} / \theta_{*}\right) j=-\llbracket d_{*} \partial_{\nu_{\Sigma}} \vartheta \rrbracket$. Adding theses identities and taking real parts yields the important relation

$$
\begin{aligned}
0 & =\operatorname{Re} \lambda\left|\rho^{1 / 2} u\right|_{2}^{2}+2\left|\mu_{*}^{1 / 2} D(u)\right|_{2}^{2}+\sigma \operatorname{Re} \lambda\left(\mathcal{A}_{\Sigma} h \mid h\right)_{\Sigma} \\
& +\theta_{*}\left(\operatorname{Re} \lambda\left|\left(\rho \kappa_{*}\right)^{1 / 2} \vartheta\right|_{2}^{2}+\left|d_{*}^{1 / 2} \nabla \vartheta\right|_{2}^{2}\right) .
\end{aligned}
$$

On the other hand, if $\beta:=\operatorname{Im} \lambda \neq 0$, then taking imaginary parts separately we get with $a=l_{*}(\vartheta \mid j)_{\Sigma}$

$$
\begin{aligned}
& 0=\beta\left|\rho^{1 / 2} u\right|_{2}^{2}-\sigma \beta\left(\mathcal{A}_{\Sigma} h \mid h\right)_{\Sigma}+\operatorname{Im} a, \\
& 0=\beta\left|\left(\rho \kappa_{*}\right)^{1 / 2} \vartheta\right|_{2}^{2}+\operatorname{Im} a / \theta_{*},
\end{aligned}
$$

hence

$$
\sigma\left(\mathcal{A}_{\Sigma} h \mid h\right)_{\Sigma}=\left|\rho^{1 / 2} u\right|_{2}^{2}-\theta_{*}\left|\left(\rho \kappa_{*}\right)^{1 / 2} \vartheta\right|_{2}^{2} .
$$

Inserting this identity into (4.8) leads to

$$
0=2 \operatorname{Re} \lambda\left|\rho^{1 / 2} u\right|_{2}^{2}+2\left|\mu_{*}^{1 / 2} D(u)\right|_{2}^{2}+\theta_{*}\left|d_{*}^{1 / 2} \nabla \vartheta\right|_{2}^{2} .
$$

This shows that if $\lambda$ is an eigenvalue of $-L$ with $\operatorname{Re} \lambda \geq 0$ then $\lambda$ is real. In fact, this identity implies $\vartheta=$ constant and $D(u)=0$, hence $j=0$ by the Stefan condition, and then $u=0$ by Korn's inequality and the no-slip condition on $\partial \Omega$, as well as $\vartheta=h=0$ by the equations for $\vartheta$ and $h$, since $\lambda \neq 0$. 
4. Suppose now that $\lambda>0$ is an eigenvalue of $-L$. Then we further have

$$
\lambda \int_{\Sigma} h d \Sigma=\int_{\Sigma}\left(u_{k} \cdot \nu_{\Sigma}-j / \rho_{k}\right) d \Sigma=-\rho_{k}^{-1} \int_{\Sigma} j d \Sigma,
$$

hence the mean values $\bar{h}$ of $h$ and $\bar{j}$ of $j$ vanish since the densities are non-equal. Moreover, the identity

$$
0=\left(l_{*} / \theta_{*}\right) \int_{\Sigma} j d \Sigma=-\int_{\Sigma} \llbracket d_{*} \partial_{\nu_{\Sigma}} \vartheta \rrbracket d \Sigma=\int_{\Omega} d_{*} \Delta \vartheta=\lambda \int_{\Omega} \kappa_{*} \rho \vartheta d x
$$

implies also $\left(\vartheta \mid \rho \kappa_{*}\right)_{L_{2}}=0$. Since $\mathcal{A}_{\Sigma}$ is positive semidefinite on functions with mean zero in case $\Sigma$ is connected, by (4.8) we obtain $u=\vartheta=h=0$, i.e. in this case there are no positive eigenvalues. On the other hand, if $\Sigma$ is disconnected, there is at least one positive eigenvalue. To prove this we need some preparations.

5. First we consider the heat problem

$$
\begin{aligned}
\rho \kappa_{*} \lambda \vartheta-d_{*} \Delta \vartheta & =0, & & x \in \Omega \backslash \Sigma, \\
\llbracket \vartheta \rrbracket & =0, & & x \in \Sigma, \\
-\llbracket d_{*} \partial_{\nu_{\Sigma}} \vartheta \rrbracket & =g, & & x \in \Sigma, \\
\partial_{\nu} \vartheta & =0, & & x \in \partial \Omega,
\end{aligned}
$$

to obtain $\vartheta=N_{\lambda}^{H} g$, where $N_{\lambda}^{H}$ denotes the Neumann-to-Dirichlet operator for this heat problem. The properties of $N_{\lambda}^{H}$ are summarized in the following proposition; see [26] for a proof. We denote by e the function which is identically one on $\Sigma$.

Proposition 4.1. The Neumann-to-Dirichlet operator $N_{\lambda}^{H}$ for the diffusion problem (4.9) admits a compact self-adjoint extension to $L_{2}(\Sigma)$ which has the following properties.

(i) If $\vartheta$ denotes the solution of (4.9), then

$$
\left(N_{\lambda}^{H} g \mid g\right)_{L_{2}(\Sigma)}=\lambda\left|\sqrt{\rho \kappa_{*}} \vartheta\right|_{L_{2}(\Omega)}^{2}+\left|\sqrt{d_{*}} \nabla \vartheta\right|_{L_{2}(\Omega}^{2}, \quad \lambda>0, g \in H_{2}^{1 / 2}(\Sigma) ;
$$

in particular, $N_{\lambda}^{H}$ is injective for $\lambda>0$.

(ii) For each $\alpha \in(0,1 / 2)$ and $\lambda_{0}>0$ there is a constant $C>0$ such that

$$
\left(N_{\lambda}^{H} g \mid g\right)_{L_{2}(\Sigma)} \geq \frac{\lambda^{\alpha}}{C}\left|N_{\lambda}^{H} g\right|_{L_{2}(\Sigma)}^{2}, \quad g \in L_{2}(\Sigma), \lambda \geq \lambda_{0} ;
$$

hence

$$
\left|N_{\lambda}^{H}\right|_{\mathcal{B}\left(L_{2}(\Sigma)\right)} \leq \frac{C}{\lambda^{\alpha}}, \quad \lambda \geq \lambda_{0} .
$$

(iii) On $L_{2,0}(\Sigma):=\left\{g \in L_{2}(\Sigma):(g \mid \mathrm{e})_{L_{2}(\Sigma)}=0\right\}$, we even have

$$
\left(N_{\lambda}^{H} g \mid g\right)_{L_{2}(\Sigma)} \geq \frac{(1+\lambda)^{\alpha}}{C}\left|N_{\lambda}^{H} g\right|_{L_{2}(\Sigma)}^{2}, \quad g \in L_{2,0}(\Sigma), \lambda>0,
$$

and

$$
\left|N_{\lambda}^{H}\right|_{\mathcal{B}\left(L_{2,0}(\Sigma)\right)} \leq \frac{C}{(1+\lambda)^{\alpha}}, \quad \lambda>0 .
$$

In particular, for $\lambda=0$, (4.9) is solvable if and only if $(g \mid e)_{L_{2}(\Sigma)}=0$, and then the solution is unique up to a constant. 
6. We also need a corresponding result for the Neumann-to-Dirichlet operator $N_{\lambda}^{S}$ for the Stokes problem. It is defined as follows. Given a function $g \in \dot{W}_{p}^{1-1 / p}(\Sigma)$, we solve the Stokes problem

$$
\begin{aligned}
\rho \lambda u-\mu_{*} \Delta u+\nabla \pi=0, & x \in \Omega \backslash \Sigma, \\
\operatorname{div} u=0, & x \in \Omega \backslash \Sigma, \\
\llbracket u \rrbracket=0, & x \in \Sigma, \\
-\llbracket T(u, \pi, \vartheta) \nu_{\Sigma} \rrbracket=g \nu_{\Sigma}, & x \in \Sigma, \\
u=0, & x \in \partial \Omega,
\end{aligned}
$$

and define $N_{\lambda}^{S} g:=u \cdot \nu_{\Sigma}$ on $\Sigma$. For this well-defined operator we have

Proposition 4.2. The Neumann-to-Dirichlet operator $N_{\lambda}^{S}$ for the Stokes problem (4.10) admits a compact self-adjoint extension to $L_{2}(\Sigma)$ which has the following properties.

(i) If $u$ denotes the solution of (4.10), then

$$
\left(N_{\lambda}^{S} g \mid g\right)_{L_{2}(\Sigma)}=\lambda \int_{\Omega} \rho|u|^{2} d x+2 \int_{\Omega} \mu_{*}|D(u)|_{2}^{2} d x, \quad \lambda \geq 0, g \in H_{2}^{1 / 2}(\Sigma) .
$$

(ii) For each $\alpha \in(0,1 / 2)$ there is a constant $C>0$ such that

$$
\left(N_{\lambda}^{S} g \mid g\right)_{L_{2}(\Sigma)} \geq \frac{(1+\lambda)^{\alpha}}{C}\left|N_{\lambda}^{S} g\right|_{L_{2}(\Sigma)}^{2}, \quad g \in L_{2}(\Sigma), \lambda \geq 0
$$

In particular,

$$
\left|N_{\lambda}^{S}\right|_{\mathcal{B}\left(L_{2}(\Sigma)\right)} \leq \frac{C}{(1+\lambda)^{\alpha}}, \quad \lambda \geq 0
$$

(iii) Let $\Sigma_{k}$ denote the components of $\Sigma$ and let $\mathrm{e}_{k}$ be the function which is one on $\Sigma_{k}$, zero elsewhere. Then $\left(N_{\lambda}^{S} g \mid \mathrm{e}_{k}\right)_{L_{2}(\Sigma)}=0$ for each $g \in L_{2}(\Sigma)$. In particular, $N_{\lambda}^{S} e_{k}=0$ for each $k$, and $N_{\lambda}^{S} g$ has mean value zero for each $g \in L_{2}(\Sigma)$.

This result is proved in [27, Proposition 4.1 for the case of equal densities. It carries over directly to the case $\llbracket \rho \rrbracket \neq 0$ considered here. Note that $N_{\lambda}^{S}$ even on $L_{2,0}(\Sigma)$ is not injective in case $\Sigma$ is disconnected.

7. Next we solve the asymmetric Stokes problem

$$
\begin{aligned}
\rho \lambda u-\mu_{*} \Delta u+\nabla \pi=0, & x \in \Omega \backslash \Sigma, \\
\operatorname{div} u=0, & x \in \Omega \backslash \Sigma, \\
P_{\Sigma} \llbracket u \rrbracket=P_{\Sigma} \llbracket T(u, \pi, \vartheta) \nu_{\Sigma} \rrbracket=0, & x \in \Sigma, \\
-\llbracket T(u, \pi, \vartheta) \nu_{\Sigma} \cdot \nu_{\Sigma} \rrbracket=g_{1}, & x \in \Sigma, \\
-\llbracket T(u, \pi, \vartheta) \nu_{\Sigma} \cdot \nu_{\Sigma} / \rho \rrbracket=g_{2}, & x \in \Sigma,
\end{aligned}
$$

to obtain as output

$$
k=\llbracket \rho u \cdot \nu_{\Sigma} \rrbracket / \llbracket \rho \rrbracket=S_{\lambda}^{11} g_{1}+S_{\lambda}^{12} g_{2}, \quad j=\llbracket u \cdot \nu_{\Sigma} \rrbracket / \llbracket 1 / \rho \rrbracket=S_{\lambda}^{21} g_{1}+S_{\lambda}^{22} g_{2} .
$$

For this problem we have 
Proposition 4.3. The operator $S_{\lambda}$ for the Stokes problem (4.11) admits a bounded extension to $L_{2,0}(\Sigma)^{2}$ for $\lambda \geq 0$ and has the following properties.

(i) If $u$ denotes the solution of (4.11), then

$\left(S_{\lambda} g \mid g\right)_{L_{2}(\Sigma)^{2}}=\lambda \int_{\Omega} \rho|u|^{2} d x+2 \int_{\Omega} \mu_{*}|D(u)|_{2}^{2} d x, \lambda \geq 0, g \in L_{2,0}(\Sigma)^{2} \cap H_{2}^{1 / 2}(\Sigma)^{2}$.

(ii) $S_{\lambda} \in \mathcal{B}\left(L_{2,0}(\Sigma)^{2}\right)$ is self-adjoint, positive semidefinite, and compact; in particular

$$
S_{\lambda}^{11}=\left[S_{\lambda}^{11}\right]^{*}, \quad S_{\lambda}^{22}=\left[S_{\lambda}^{22}\right]^{*}, \quad S_{\lambda}^{12}=\left[S_{\lambda}^{21}\right]^{*} .
$$

(iii) $S_{\lambda}^{11}$ and $S_{\lambda}^{22}$ are injective in $L_{2,0}(\Sigma)$, and with $G_{\lambda}=\left[S_{\lambda}^{22}\right]^{-1}$ we have

$$
N_{\lambda}^{S}=S_{\lambda}^{11}-S_{\lambda}^{12} G_{\lambda} S_{\lambda}^{21} \text {. }
$$

$G_{\lambda}$ is self-adjoint and positive definite on $L_{2,0}(\Sigma)$, its resolvent is compact in $L_{2,0}(\Sigma)$, for each $\lambda \geq 0$.

(iv) For each $\beta \in(0,1 / 2)$ there is a constant $C_{\beta}>0$ such that

$$
\left|S_{\lambda}\right|_{\mathcal{B}\left(L_{2,0}(\Sigma)^{2}\right)} \leq \frac{C_{\beta}}{(1+\lambda)^{\beta}}, \quad \lambda \geq 0 .
$$

(v) $\left|S_{\lambda}\right|_{\mathcal{B}\left(L_{2,0}(\Sigma)^{2}, H_{2}^{1}(\Sigma)^{2}\right)} \leq C$ uniformly for $\lambda \geq 0$.

(vi) $S_{\lambda}^{11}, S_{\lambda}^{22}: L_{2,0}(\Sigma) \rightarrow H_{2}^{1}(\Sigma) \cap L_{2,0}(\Sigma)$ are isomorphisms, for each $\lambda \geq 0$.

Proof. (a) First observe that for the traces $u_{j}$ of $u$ on $\Sigma$ we have

$$
u_{j}=P_{\Sigma} u+k \nu_{\Sigma}+j \nu_{\Sigma} / \rho_{j}=u_{b}+j \nu_{\Sigma} / \rho_{j} \quad \text { on } \Sigma .
$$

To prove assertion (i), let $(u, \pi)$ denote the solution of (4.11). Multiply with $u$ and integrate by parts to the result

$$
\begin{aligned}
\lambda \int_{\Omega} \rho|u|^{2} d x & +2 \int_{\Omega} \mu_{*}|D(u)|_{2}^{2} d x=\int_{\Omega} \operatorname{div}(T(u, \pi, \vartheta) \bar{u}) d x \\
& =-\int_{\Sigma} \llbracket \bar{u} \cdot T(u, \pi, \vartheta) \nu_{\Sigma} \rrbracket d \Sigma=-\int_{\Sigma} \llbracket \overline{\left(u_{b}+j \nu_{\Sigma} / \rho\right)} \cdot T(u, \pi, \vartheta) \nu_{\Sigma} \rrbracket d \Sigma \\
& =-\int_{\Sigma} \overline{u_{b}} \cdot \llbracket T(u, \pi, \vartheta) \nu_{\Sigma} \rrbracket d \Sigma-\int_{\Sigma} \bar{j} \llbracket T(u, \pi, \vartheta) \nu_{\Sigma} \cdot \nu_{\Sigma} / \rho \rrbracket d \Sigma \\
& =\int_{\Sigma} \bar{k} g_{1} d \Sigma+\int_{\Sigma} \bar{j} g_{2} d \Sigma=\left(g \mid S_{\lambda} g\right)_{L_{2}(\Sigma)^{2}} .
\end{aligned}
$$

A similar computation yields

$$
\left(S_{\lambda} g \mid h\right)_{L_{2}(\Sigma)^{2}}=\left(g \mid S_{\lambda} h\right)_{L_{2}(\Sigma)^{2}},
$$

hence $S_{\lambda}$ is self-adjoint in $L_{2}(\Sigma)^{2}$, thereby proving the first part of (ii).

(b) To prove injectivity of $S_{\lambda}^{22}$, let $g_{1}=0$ and $j=0$. Then (i) implies $\lambda u=$ $D(u)=0$ hence $\nabla \pi=0$ in $\Omega \backslash \Sigma$, and so $\pi$ is constant in the components of the phases. Next

$$
0=g_{1}=-\llbracket T(u, \pi, \vartheta) \nu_{\Sigma} \cdot \nu_{\Sigma} \rrbracket=\llbracket \pi \rrbracket, \quad g_{2}=-\llbracket T(u, \pi, \vartheta) \nu_{\Sigma} \cdot \nu_{\Sigma} / \rho \rrbracket=\llbracket \pi / \rho \rrbracket
$$


shows that $\pi$ is even constant in the phases, and so $g_{2}$ is constant on $\Sigma$ hence zero since its mean value vanishes. Injectivity of $S_{\lambda}^{11}$ is shown in a similar way. This proves the first assertion in (iii), the second one follows from the definitions of $N_{\lambda}^{S}$ and $S_{\lambda}$, and the third one is a consequence of (ii).

(c) To establish the boundedness properties of $S_{\lambda}$, we proceed as follows. Suppose $g_{1}, g_{2} \in L_{2,0}(\Sigma) \cap H_{2}^{1 / 2}(\Sigma)$ are given. We decompose the pressure into $\pi=q+q_{0}$, where the one-sided traces $q_{0}^{j}$ are uniquely determined on $\Sigma$ by

$$
\llbracket q_{0} \rrbracket=g_{1}, \quad \llbracket q_{0} / \rho \rrbracket=g_{2} ;
$$

then $q_{0}^{j} \in L_{2,0}(\Sigma)$. Extend $q_{0}$ to all of $\Omega$ in $H_{2}^{1 / 2}(\Omega \backslash \Sigma)$, by solving the problem

$$
\Delta v=0 \text { in } \Omega \backslash \Sigma, \quad v=q_{0} \text { on } \Sigma, \quad v=0 \text { on } \partial \Omega .
$$

There is a constant $c_{0}>0$ such that

$$
\left|q_{0}\right|_{H_{2}^{1 / 2}(\Omega \backslash \Sigma)} \leq c_{0}|g|_{L_{2}(\Sigma)}
$$

Then $(u, q)$ satisfies the asymmetric Stokes problem

$$
\begin{array}{rlrl}
\rho \lambda u-\mu_{*} \Delta u+\nabla q=-\nabla q_{0}, & & x \in \Omega \backslash \Sigma, \\
\operatorname{div} u & =0, & & x \in \Omega \backslash \Sigma, \\
P_{\Sigma} \llbracket u \rrbracket=P_{\Sigma} \llbracket \mu_{*} D(u) \nu_{\Sigma} \rrbracket & =0, & & x \in \Sigma, \\
-2 \llbracket \mu_{*} D(u) \nu_{\Sigma} \cdot \nu_{\Sigma} \rrbracket+\llbracket q \rrbracket & =0, & & x \in \Sigma, \\
-2 \llbracket \mu_{*} D(u) \nu_{\Sigma} \cdot \nu_{\Sigma} / \rho \rrbracket+\llbracket q / \rho \rrbracket & =0, & & x \in \Sigma, \\
u & =0, & & x \in \partial \Omega .
\end{array}
$$

Note that the interface conditions are now homogeneous. Let $-A$ denote the generator of the associated analytic $C_{0}$-semigroup in $L_{2, \sigma}(\Omega)$, which is exponentially stable. Then we have

$$
u_{\lambda}:=-(\lambda+A)^{-1}\left[\nabla q_{0} / \rho\right]
$$

and with

$$
S_{\lambda} g=(k, j)=\left(\llbracket \rho u_{\lambda} \cdot \nu_{\Sigma} \rrbracket / \llbracket \rho \rrbracket, \llbracket u_{\lambda} \cdot \nu_{\Sigma} \rrbracket / \llbracket 1 / \rho \rrbracket\right)
$$

we estimate using trace theory and the resolvent estimate for $A$ and $\operatorname{Re} \sigma(-A)<0$

$$
\left|S_{\lambda} g\right|_{H_{2}^{1}(\Sigma)} \leq C\left|u_{\lambda}\right|_{H_{2}^{3 / 2}(\Omega \backslash \Sigma)} \leq C\left|q_{0}\right|_{H_{2}^{1 / 2}(\Omega \backslash \Sigma)} \leq C|g|_{L_{2}(\Sigma)},
$$

as well as

$$
\left|S_{\lambda} g\right|_{L_{2}(\Sigma)} \leq C\left|u_{\lambda}\right|_{H_{2}^{\alpha}(\Omega \backslash \Sigma)} \leq C(1+\lambda)^{\beta-1}\left|q_{0}\right|_{H_{2}^{1 / 2}(\Omega \backslash \Sigma)} \leq C(1+\lambda)^{\beta-1}|g|_{L_{2}(\Sigma)},
$$

with $\beta=(\alpha+1 / 2) / 2$, for each fixed $\alpha>1 / 2$. These estimates are uniform w.r.t. $\lambda \geq 0$. 
(d) The proof of (vi) is more involved. To obtain surjectivity of $S_{\lambda}^{11}$ we have to solve the problem

$$
\begin{aligned}
\rho \lambda u-\mu_{*} \Delta u+\nabla \pi=0, & x \in \Omega \backslash \Sigma, \\
\operatorname{div} u=0, & x \in \Omega \backslash \Sigma, \\
P_{\Sigma} \llbracket u \rrbracket=P_{\Sigma} \llbracket T(u, \pi, \vartheta) \nu_{\Sigma} \rrbracket=0, & x \in \Sigma, \\
\llbracket \rho u \cdot \nu_{\Sigma} \rrbracket=\llbracket \rho \rrbracket k, & x \in \Sigma, \\
-\llbracket T(u, \pi, \vartheta) \nu_{\Sigma} \cdot \nu_{\Sigma} / \rho \rrbracket=0, & x \in \Sigma, \\
u=0, & x \in \partial \Omega,
\end{aligned}
$$

for given $k \in H_{2}^{1}(\Sigma) \cap L_{2,0}(\Sigma)$ with output $\left[S_{\lambda}^{11}\right]^{-1} k=g_{1}=-\llbracket T(u, \pi, \vartheta) \nu_{\Sigma} \cdot \nu_{\Sigma} \rrbracket$. Similarly, to prove surjectivity of $S_{\lambda}^{22}$ the problem to solve is

$$
\begin{array}{rl}
\rho \lambda u-\mu_{*} \Delta u+\nabla \pi=0, & x \in \Omega \backslash \Sigma, \\
\operatorname{div} u=0, & x \in \Omega \backslash \Sigma, \\
P_{\Sigma} \llbracket u \rrbracket=P_{\Sigma} \llbracket T(u, \pi, \vartheta) \nu_{\Sigma} \rrbracket=0, & x \in \Sigma, \\
\llbracket u \cdot \nu_{\Sigma} \rrbracket=\llbracket 1 / \rho \rrbracket j & x \in \Sigma, \\
-\llbracket T(u, \pi, \vartheta) \nu_{\Sigma} \cdot \nu_{\Sigma} \rrbracket=0, & x \in \Sigma, \\
u=0, & x \in \partial \Omega,
\end{array}
$$

for given $j$ and the output will be $\left[S_{\lambda}^{22}\right]^{-1} j=g_{2}=-\llbracket T(u, \pi, \vartheta) \nu_{\Sigma} \cdot \nu_{\Sigma} / \rho \rrbracket$. These problems can be solved in a similar way as in the proof of Theorem 3.1. Using the method of localization and perturbation we reduce the problems to the case of a flat interface, i.e. $\Omega=\mathbb{R}^{n}, \Sigma=\mathbb{R}^{n-1} \times\{0\}$. Then we may employ a partial Fourier-transform in the same way as in the proof of [23, Theorem 3.1.

8. Now suppose that $\lambda>0$ is an eigenvalue of $L$. We set $g=l_{*} j / \theta_{*}, g_{1}=-\sigma \mathcal{A}_{\Sigma} h$ and $g_{2}=-l_{*} \vartheta=-c_{*} N_{\lambda}^{H} j, c_{*}=l_{*}^{2} / \theta_{*}>0$ to obtain

$$
j=-S_{\lambda}^{21} \sigma \mathcal{A}_{\Sigma} h-c_{*} S_{\lambda}^{22} N_{\lambda}^{H} j, \quad \lambda h=-S_{\lambda}^{11} \sigma \mathcal{A}_{\Sigma} h-S_{\lambda}^{12} c_{*} N_{\lambda}^{H} j .
$$

Observing that $I+c_{*} S_{\lambda}^{22} N_{\lambda}^{H}$ is injective, hence boundedly invertible in $L_{2,0}(\Sigma)$ by compactness of $S_{\lambda}$, we may solve the first equation for $j$ to the result

$$
j=-\sigma\left(I+c_{*} S_{\lambda}^{22} N_{\lambda}^{H}\right)^{-1} S_{\lambda}^{21} \mathcal{A}_{\Sigma} h .
$$

Inserting this into the second, the equation for $h$ becomes

$$
0=\lambda h+\sigma\left(S_{\lambda}^{11}-S_{\lambda}^{12} c_{*} N_{\lambda}^{H}\left(I+c_{*} S_{\lambda}^{22} N_{\lambda}^{H}\right)^{-1} S_{\lambda}^{21}\right) \mathcal{A}_{\Sigma} h,
$$

or equivalently with $R_{\lambda}=G_{\lambda} S_{\lambda}^{21}$

$$
0=\lambda h+\sigma\left(N_{\lambda}^{S}+R_{\lambda}^{*}\left(c_{*} N_{\lambda}^{H}+G_{\lambda}\right)^{-1} R_{\lambda}\right) \mathcal{A}_{\Sigma} h .
$$

Next we observe that the operators $N_{\lambda}^{S}+R_{\lambda}^{*}\left(c_{*} N_{\lambda}^{H}+G_{\lambda}\right)^{-1} R_{\lambda}$ are injective for $\lambda \geq 0$; in fact if

$$
N_{\lambda}^{S} h+R_{\lambda}^{*}\left(c_{*} N_{\lambda}^{H}+G_{\lambda}\right)^{-1} R_{\lambda} h=0,
$$


then with $v_{\lambda}=\left(c_{*} N_{\lambda}^{H}+G_{\lambda}\right)^{-1} R_{\lambda} h$, forming the inner product with $h$ in $L_{2,0}(\Sigma)$ we obtain

$$
\begin{aligned}
0 & =\left(N_{\lambda}^{S} h \mid h\right)_{\Sigma}+\left(R_{\lambda}^{*}\left(c_{*} N_{\lambda}^{H}+G_{\lambda}\right)^{-1} R_{\lambda} h \mid h\right)_{\Sigma} \\
& \geq c\left|N_{\lambda}^{S} h\right|_{\Sigma}^{2}+\left(\left(c_{*} N_{\lambda}^{H}+G_{\lambda}\right) v_{\lambda} \mid v_{\lambda}\right)_{\Sigma}
\end{aligned}
$$

hence $N_{\lambda}^{S} h=0$ and $v_{\lambda}=0$, which implies $S_{\lambda}^{21} h=S_{\lambda}^{11} h=0$, hence $h=0$ by injectivity of $S_{\lambda}^{11}$. Setting now

$$
T_{\lambda}=\left[N_{\lambda}^{S}+R_{\lambda}^{*}\left(c_{*} N_{\lambda}^{H}+G_{\lambda}\right)^{-1} R_{\lambda}\right]^{-1},
$$

we arrive at the equation

$$
\lambda T_{\lambda} h+\sigma \mathcal{A}_{\Sigma} h=0 .
$$

$\lambda>0$ is an eigenvalue of $-L$ if and only if the problem (4.15) admits a nontrivial solution, i.e. if and only if 0 is an eigenvalue for $B_{\lambda}:=\lambda T_{\lambda}+\sigma \mathcal{A}_{\Sigma}$. Here the domain of $B_{\lambda}$ is that of $\mathcal{A}_{\Sigma}, T_{\lambda}$ is a relatively compact perturbation of $\mathcal{A}_{\Sigma}$.

We consider this problem in $L_{2,0}(\Sigma)$. Then $\mathcal{A}_{\Sigma}$ is selfadjoint and

$$
\sigma\left(\mathcal{A}_{\Sigma} h \mid h\right)_{\Sigma} \geq-\frac{\sigma(n-1)}{R^{2}}|h|_{\Sigma}^{2} .
$$

On the other hand, $N_{\lambda}^{H}, N_{\lambda}^{S}$ are self-adjoint, compact and positive semidefinite on $L_{2,0}(\Sigma)$, and $G_{\lambda}$ is self-adjoint, positive definite, and has compact inverse. Hence $T_{\lambda}$ is self-adjoint, positive semidefinite and $T_{\lambda}^{-1}$ is compact as well. If $\mu>0$ is an eigenvalue of $T_{\lambda}$ then

$$
\mu^{-1} h=T_{\lambda}^{-1} h=\left[S_{\lambda}^{11}-c_{*} S_{\lambda}^{12} N_{\lambda}^{H}\left(I+c_{*} S_{\lambda}^{22} N_{\lambda}^{H}\right)^{-1} S_{\lambda}^{21}\right] h,
$$

hence we get

$$
\mu^{-1}|h|_{\Sigma} \leq C|h|_{\Sigma}
$$

since by Propositions 4.3 and $4.1 S_{\lambda}, N_{\lambda}^{H}$ and $\left(I+c_{*} S_{\lambda}^{22} N_{\lambda}^{H}\right)^{-1}$ are bounded in $L_{2,0}(\Sigma)$, uniformly for large $\lambda$. Therefore $\mu=\mu(\lambda) \geq c_{0}>0$, for large $\lambda$, and so

$$
\left(B_{\lambda} h \mid h\right)_{\Sigma}=\lambda\left(T_{\lambda} h \mid h\right)_{\Sigma}+\sigma\left(\mathcal{A}_{\Sigma} h \mid h\right)_{\Sigma} \geq\left(c_{0} \lambda-\frac{\sigma(n-1)}{R^{2}}\right)|h|_{\Sigma}^{2} .
$$

This proves that $B_{\lambda}$ is positive definite, hence (4.15) has no nontrivial solution, for large $\lambda$.

But for small $\lambda>0$ we have with $h=\sum_{k=1}^{m} \chi_{\Sigma_{k}} h_{k}$, where $\chi_{A}$ denotes characteristic function of a set $A, h_{k}=$ const. on $\Sigma_{k}, \sum_{k=1}^{m} h_{k}=0$,

$$
\lambda\left(T_{\lambda} h \mid h\right)-\frac{\sigma(n-1)}{R^{2}} \omega_{n} R^{n-1} \sum_{k} h_{k}^{2}<0,
$$

since with $h \in H_{2}^{3 / 2}(\Sigma) \cap L_{2,0}(\Sigma)$

$$
\begin{aligned}
T_{\lambda} h & =\left(I-c_{*}\left[S_{\lambda}^{11}\right]^{-1} S_{\lambda}^{12} N_{\lambda}^{H}\left(I+c_{*} S_{\lambda}^{22} N_{\lambda}^{H}\right)^{-1} S_{\lambda}^{21}\right)^{-1} \\
& :=\left(I-K_{\lambda}\right)^{-1}\left[S_{\lambda}^{11}\right]^{-1} h \rightarrow T_{0} h
\end{aligned}
$$

in $L_{2,0}(\Sigma)$ as $\lambda \rightarrow 0$. This can seen from the mapping and continuity properties of $S_{\lambda}$ and $N_{\lambda}^{H} ; K_{\lambda}$ is compact and continuous in $\mathcal{B}\left(L_{2,0}(\Sigma)\right)$, and $I-K_{\Lambda}$ is injective, 
hence invertible and its inverse is continuous as well. This shows that $B_{\lambda}$ has a nontrivial kernel for some $\lambda_{0}>0$, which implies that $-L$ has a positive eigenvalue.

Even more is true. We have seen that $B_{\lambda}$ is positive definite for large $\lambda$ and $B_{0}=\sigma \mathcal{A}_{\Sigma}$ has $-\sigma(n-1) / R_{*}^{2}$ as an eigenvalue of multiplicity $m-1$ in $L_{2,0}(\Sigma)$. Therefore, as $\lambda$ increases to infinity, $m-1$ eigenvalues $\mu_{k}(\lambda)$ of $B_{\lambda}$ must cross through zero, this way inducing $m-1$ positive eigenvalues of $-L$.

9. Next we look at the eigenvalue $\lambda=0$. Then (4.8) yields

$$
2\left|\mu_{*}^{1 / 2} D(u)\right|_{2}^{2}+\left|d_{*}^{1 / 2} \nabla \vartheta\right|_{2}^{2}=0,
$$

hence $\vartheta$ is constant, $D(u)=0$ and $j=0$ by the flux condition for $\vartheta$. This further implies that $\llbracket u \rrbracket=0$, and therefore Korn's inequality yields $\nabla u=0$ and then we have $u=0$ by the no-slip condition on $\partial \Omega$. This implies further that the pressures are constant in the phases and $\llbracket \pi \rrbracket=-\sigma \mathcal{A}_{\Sigma} h$, as well as $l_{*} \vartheta=-\llbracket \pi / \rho \rrbracket$. Thus the dimension of the eigenspace for eigenvalue $\lambda=0$ is the same as the dimension of the manifold of equilibria, namely $m \cdot n+2$ if $\Omega_{1}$ has $m \geq 1$ components. The kernel of $L$ is spanned by $e_{\theta}=(0,1,0), e_{h}=(0,0,1), e_{j k}=\left(0,0, Y_{j}^{k}\right)$ with the spherical harmonics $Y_{j}^{k}$ of degree one for the spheres $\Sigma^{k}, j=1, \ldots, n, k=1, \ldots, m$.

To show that the equilibria are normally stable or hyperbolic, it remains to prove that $\lambda=0$ is semi-simple. So suppose we have a solution of $L(u, \vartheta, h)=$ $\sum_{j, k} \alpha_{j k} e_{j k}+\beta e_{\theta}+\gamma e_{h}$. This means

$$
\begin{aligned}
-\mu_{*} \Delta u+\nabla \pi=0, & x \in \Omega \backslash \Sigma, \\
\operatorname{div} u=0, & x \in \Omega \backslash \Sigma, \\
\llbracket u \rrbracket=\llbracket \rho^{-1} \rrbracket j \nu_{\Sigma}, & x \in \Sigma, \\
-\llbracket T(u, \pi, \vartheta) \nu_{\Sigma} \rrbracket=-\sigma \mathcal{A}_{\Sigma} h \nu_{\Sigma}, & x \in \Sigma, \\
u=0, & x \in \partial \Omega . \\
-d_{*} \Delta \vartheta=\beta, & x \in \Omega \backslash \Sigma, \\
\llbracket \vartheta \rrbracket=0, & x \in \Sigma, \\
-\left(l_{*} / \theta_{*}\right) j-\llbracket d_{*} \partial_{\nu_{\Sigma}} \vartheta \rrbracket=0, & x \in \Sigma, \\
\partial_{\nu} \vartheta=0, & x \in \partial \Omega . \\
\llbracket-\rho^{-1} T(u, \pi, \vartheta) \nu_{\Sigma} \cdot \nu_{\Sigma} \rrbracket+l_{*} \vartheta=0, & x \in \Sigma, \\
-\llbracket \rho u \cdot \nu_{\Sigma} \rrbracket / \llbracket \rho \rrbracket=\sum_{j, k} \alpha_{j} Y_{j}^{k}+\gamma, & t>0, x \in \Sigma .
\end{aligned}
$$

We have to show $\alpha_{j k}=\beta=\gamma=0$ for all $j$. Integrating the divergence equation for $u$ over $\Omega$ we get $\int_{\Sigma} j d \Sigma=0$, and integrating that for $\vartheta$ yields

$$
\beta|\Omega|=-\int_{\Omega} d_{*} \Delta \vartheta d x=\int_{\Sigma} \llbracket d_{*} \partial_{\nu_{\Sigma}} \vartheta \rrbracket d \Sigma=-\left(l_{*} / \theta_{*}\right) \int_{\Sigma} j d \Sigma=0,
$$


which implies $\beta=0$. As in 3. we then obtain

$$
2\left|\mu_{*}^{1 / 2} D(u)\right|_{2}^{2}+\left(\llbracket \rho^{-1} T(u, \pi, \vartheta) \nu_{\Sigma} \cdot \nu_{\Sigma} \rrbracket \mid j\right)_{\Sigma}=0
$$

and

$$
\theta_{*}\left|d_{*}^{1 / 2} \nabla \vartheta\right|_{2}^{2}-\left(j \mid \llbracket \rho^{-1} T(u, \pi, \vartheta) \nu_{\Sigma} \cdot \nu_{\Sigma} \rrbracket\right)_{\Sigma}=\beta(\vartheta \mid 1)_{\Omega}
$$

Adding these equations yields

$$
2\left|\mu_{*}^{1 / 2} D(u)\right|_{2}^{2}+\theta_{*}\left|d_{*}^{1 / 2} \nabla \vartheta\right|_{2}^{2}=0,
$$

This implies $D(u)=0, \vartheta$ constant, hence $j=0$ since $l_{*} \neq 0$, and then $u=0$ by Korn's inequality, which in turn yields

$$
0=-\llbracket \rho u \cdot \nu_{\Sigma} \rrbracket / \llbracket \rho \rrbracket=\sum_{j, k} \alpha_{j k} Y_{j}^{k}+\gamma .
$$

Thus $\gamma=\alpha_{j k}=0$ for all $j, k$ since the spherical harmonics $Y_{j}^{k}$ are independent and have mean zero. Therefore eigenvalue $\lambda=0$ is semi-simple.

10. Let us consider now the case $l_{*}=0$. Then the temperature equation decouples completely from that for $u$ and $h$. It only induces one dimension in the kernel of $L$, but no positive eigenvalues. In this case, as now $c_{*}=0$ the derivation in 8 . yields the equivalent problem

$$
\lambda h+\sigma S_{\lambda}^{11} \mathcal{A}_{\Sigma} h=0 .
$$

As $S_{\lambda}^{11}$ is positive semidefinite and injective, this equation admits no nontrivial solutions if $\operatorname{Re} \lambda \geq$ and $\lambda$ is non-real. If $\lambda>0$ then $T_{\lambda}=\left[S_{\lambda}^{11}\right]^{-1}$ and may employ the same arguments as in 8 . to obtain the same conclusions as in case $l_{*} \neq 0$. Finally, for $\lambda=0$ we have the same kernel of $L$ as before, and 0 is semi-simple for $L$, too.

11. Let us summarize what we have proved.

Theorem 4.4. Let $L$ denote the linearization at $e_{*}:=\left(0, \theta_{*}, \Sigma\right) \in \mathcal{E}$ as defined above. Then $-L$ generates a compact analytic $C_{0}$-semigroup in $X_{0}$ which has maximal $L_{p}$-regularity. The spectrum of $L$ consists only of eigenvalues of finite algebraic multiplicity. Moreover, the following assertions are valid.

(i) The operator $-L$ has no eigenvalues $\lambda \neq 0$ with nonnegative real part if and only if $\Sigma$ is connected.

(ii) If $\Sigma$ is disconnected, then $-L$ has precisely $m-1$ positive eigenvalues.

(iii) $\lambda=0$ is an eigenvalue of $L$ and it is semi-simple.

(iv) The kernel $N(L)$ of $L$ is isomorphic to the tangent space $T_{e_{*}} \mathcal{E}$ of the manifold of equilibria $\mathcal{E}$ at $e_{*}$.

Consequently, $e_{*}=\left(0, \theta_{*}, \Sigma\right) \in \mathcal{E}$ is normally stable if and only if $\Sigma$ is connected, and normally hyperbolic if and only if $\Sigma$ is disconnected. 


\section{Nonlinear Stability of Equilibria}

1. We look at Problem (1.1), (1.2), (1.3) in the neighborhood of a non-degenerate equilibrium $e_{*}=\left(0, \theta_{*}, \Gamma_{*}\right) \in \mathcal{E}$. Performing a Hanzawa transform with reference manifold $\Sigma=\Gamma_{*}$ as in Section 3, the transformed problem becomes

$$
\begin{aligned}
\rho \partial_{t} u-\mu_{*} \Delta u+\nabla \pi & =\rho F_{u}(u, \vartheta, h, \pi), & & \text { in } \Omega \backslash \Sigma, \\
\operatorname{div} u & =G_{d}(u, h), & & \text { in } \Omega \backslash \Sigma, \\
P_{\Sigma} \llbracket u \rrbracket & =G_{u}(u, \vartheta, h), & & \text { on } \Sigma, \\
-P_{\Sigma} \llbracket \mu_{*}\left(\nabla u+[\nabla u]^{\top}\right) \rrbracket \nu_{\Sigma} & =G_{\tau}(u, \vartheta, h), & & \text { on } \Sigma, \\
-\llbracket T(u, \pi, \vartheta) \nu_{\Sigma} \cdot \nu_{\Sigma} \rrbracket+\sigma \mathcal{A}_{\Sigma} h & =G_{\nu}(u, \vartheta, h)+G_{\gamma}(h), & & \text { on } \Sigma, \\
l_{*} \vartheta-\llbracket T(u, \pi, \vartheta) \nu_{\Sigma} \cdot \nu_{\Sigma} / \rho \rrbracket & =G_{h}(u, \vartheta, h), & & \text { on } \Sigma, \\
u & =0, & & \text { on } \partial \Omega, \\
u(0) & =u_{0}, & & \text { in } \Omega,
\end{aligned}
$$

where $\mu_{*}=\mu\left(\theta_{*}\right)>0$ and $\mathcal{A}_{\Sigma}=-H^{\prime}(0)=-(n-1) / R_{*}^{2}-\Delta_{\Sigma}$.

For the relative temperature $\vartheta=\left(\theta-\theta_{*}\right) / \theta_{*}$ we obtain

$$
\begin{aligned}
\rho \kappa_{*} \partial_{t} \vartheta-d_{*} \Delta \vartheta & =F_{\theta}(u, \vartheta, h), & & \text { in } \Omega \backslash \Sigma, \\
\llbracket \vartheta \rrbracket & =0, & & \text { on } \Sigma, \\
-\left(l_{*} / \theta_{*}\right) \llbracket u \cdot \nu_{\Sigma} \rrbracket / \llbracket 1 / \rho \rrbracket-\llbracket d_{*} \partial_{\nu_{\Sigma}} \vartheta \rrbracket & =G_{\theta}(u, \vartheta, h), & & \text { on } \Sigma, \\
\partial_{\nu} \vartheta & =0, & & \text { on } \partial \Omega, \\
\vartheta(0) & =\vartheta_{0}, & & \text { in } \Omega,
\end{aligned}
$$

with $\kappa_{*}=\kappa\left(\theta_{*}\right)>0$ and $l_{*}=l\left(\theta_{*}\right) \neq 0$. Finally, the evolution of $h$ is determined by

$$
\begin{aligned}
\partial_{t} h-\llbracket \rho u \cdot \nu_{\Sigma} \rrbracket / \llbracket \rho \rrbracket & =F_{h}(u, h), & & \text { on } \Sigma, \\
h(0) & =h_{0} . & &
\end{aligned}
$$

Here the nonlinearities are of class $C^{1}$ from $\mathbb{E}$ to $\mathbb{F}$, and satisfy $F_{j}^{\prime}(0)=G_{k}^{\prime}(0)=0$ for all $j=u, \theta, h$ and $k=d, u, \tau, \nu, \gamma, h, \theta$. Let $w:=(z, \pi):=(u, \vartheta, h, \pi)$ and $z_{0}:=\left(u_{0}, \vartheta_{0}, h_{0}\right)$. We will frequently make use of the shorter notation

$$
\mathbb{L} w=N(w), \quad z(0)=z_{0}
$$

for the system (5.1)-(5.3).

The state manifold locally near the equilibrium $e_{*}=\left(0, \theta_{*}, \Gamma_{*}\right)$ reads as

$$
\begin{aligned}
\mathcal{S M}:= & \left\{(u, \vartheta, h) \in L_{p}(\Omega)^{n+1} \times C^{2}(\Sigma):(u, \vartheta) \in W_{p}^{2-2 / p}(\Omega \backslash \Sigma)^{n+1}, h \in W_{p}^{3-2 / p},\right. \\
& P_{\Sigma} \llbracket u \rrbracket=G_{u}(u, \vartheta, h),-P_{\Sigma} \llbracket \mu_{*}\left(\nabla u+[\nabla u]^{\top}\right) \rrbracket \nu_{\Sigma}=G_{\tau}(u, \vartheta, h) \text { on } \Sigma, \\
& \operatorname{div} u=G_{d}(u, h) \text { in } \Omega \backslash \Sigma, \quad u=\partial_{\nu} \theta=0 \text { on } \partial \Omega, \\
& \left.\llbracket \vartheta \rrbracket=0,-\left(l_{*} / \theta_{*}\right) \llbracket u \cdot \nu_{\Sigma} \rrbracket / \llbracket 1 / \rho \rrbracket-\llbracket d_{*} \partial_{\nu_{\Sigma}} \vartheta \rrbracket=G_{\theta}(u, \vartheta, h) \text { on } \Sigma\right\} .
\end{aligned}
$$


Note that due to the compatibility conditions this is a nonlinear manifold. We need to parameterize this manifold over its tangent space

$$
\begin{aligned}
& \mathcal{S} \mathcal{X}:=\{((u, \vartheta, h) \in L_{p}(\Omega)^{n+1} \times C^{2}(\Sigma):(u, \vartheta) \in W_{p}^{2-2 / p}(\Omega \backslash \Sigma)^{n+1}, h \in W_{p}^{3-2 / p}, \\
& P_{\Sigma} \llbracket u \rrbracket=0,-P_{\Sigma} \llbracket \mu_{*}\left(\nabla u+[\nabla u]^{\top}\right) \rrbracket \nu_{\Sigma}=0 \text { on } \Sigma, \\
& \operatorname{div} u=0 \text { in } \Omega \backslash \Sigma, \quad u=\partial_{\nu} \theta=0 \text { on } \partial \Omega, \\
&\left.\llbracket \vartheta \rrbracket=0,-\left(l_{*} / \theta_{*}\right) \llbracket u \cdot \nu_{\Sigma} \rrbracket / \llbracket 1 / \rho \rrbracket-\llbracket d_{*} \partial_{\nu_{\Sigma}} \vartheta \rrbracket=0 \text { on } \Sigma\right\} .
\end{aligned}
$$

We mention that the norm in $\mathcal{S X}$ is given by

$$
|(u, \vartheta, h)|_{\mathcal{S X}}=|u|_{W_{p}^{2-2 / p}}+|\vartheta|_{W_{p}^{2-2 / p}}+|h|_{W_{p}^{3-2 / p}}
$$

2. To parameterize the state manifold $\mathcal{S} \mathcal{M}$ over $\mathcal{S} \mathcal{X}$ near the given equilibrium we consider the linear elliptic problem

$$
\begin{aligned}
\rho \omega u-\mu_{*} \Delta u+\nabla \pi & =0 & & \text { in } \Omega \backslash \Sigma, \\
\operatorname{div} u & =g_{d} & & \text { in } \Omega \backslash \Sigma, \\
\rho \kappa_{*} \omega \vartheta-d_{*} \Delta \vartheta & =0 & & \text { in } \Omega \backslash \Sigma, \\
u=\partial_{\nu} \vartheta & =0 & & \text { on } \partial \Omega, \\
P_{\Sigma} \llbracket u \rrbracket & =g_{u} & & \text { on } \Sigma, \\
-P_{\Sigma} \llbracket \mu_{*}\left(\nabla u+[\nabla u]^{\top}\right) \rrbracket \nu_{\Sigma} & =g_{\tau} & & \text { on } \Sigma, \\
\llbracket \vartheta \rrbracket & =0 & & \text { on } \Sigma, \\
-\left(l_{*} / \theta_{*}\right) \llbracket u \cdot \nu_{\Sigma} \rrbracket / \llbracket 1 / \rho \rrbracket-\llbracket d_{*} \partial_{\nu_{\Sigma}} \vartheta \rrbracket & =g_{\theta} & & \text { on } \Sigma, \\
-\llbracket T(u, \pi, \vartheta) \nu_{\Sigma} \cdot \nu_{\Sigma} \rrbracket & =g_{\nu} & & \text { on } \Sigma, \\
l_{*} \vartheta-\llbracket T(u, \pi, \vartheta) \nu_{\Sigma} \cdot \nu_{\Sigma} / \rho \rrbracket & =0 & & \text { on } \Sigma,
\end{aligned}
$$

for given data $g_{d}, g_{u}, g_{\tau}, g_{\nu}, g_{\theta}$. For this problem we have the following result.

Proposition 5.1. Suppose $p>3$ and let $\omega>0$ be sufficiently large. Then problem (5.6) admits a unique solution $(u, \pi, \vartheta, h)$ with regularity

$$
(u, \vartheta) \in W_{p}^{2-2 / p}(\Omega \backslash \Sigma)^{n+1}, \quad \pi \in W_{p}^{1-2 / p}(\Omega \backslash \Sigma),
$$

if and only if the data $\left(g_{d}, g_{u}, g_{\tau}, g_{\nu}, g_{\theta}\right)$ satisfy $g_{u} \cdot \nu_{\Sigma}=g_{\tau} \cdot \nu_{\Sigma} \equiv 0$ and $g_{d} \in W_{p}^{1-2 / p}(\Omega \backslash \Sigma) \cap \dot{H}_{p}^{-1}(\Omega), \quad\left(g_{\tau}, g_{\nu}, g_{\theta}\right) \in W_{p}^{1-3 / p}(\Sigma)^{n+2}, \quad g_{u} \in W_{p}^{2-3 / p}(\Sigma)$. The solution map $\left(f_{d}, g_{u}, g_{\tau}, g_{\nu}, g_{\theta}\right) \mapsto(u, \pi, \vartheta)$ is continuous in the corresponding spaces.

This purely elliptic problem can be solved in the same way as the corresponding linear parabolic problems. 
3. For the parametrization, fix any large $\omega>0$. Given $(\tilde{u}, \tilde{\vartheta}, \tilde{h}) \in \mathcal{S} \mathcal{X}$ sufficiently small, and setting $(u, \vartheta, h)=(\tilde{u}, \tilde{\vartheta}, \tilde{h})+(\bar{u}, \bar{\vartheta}, 0)$, solve the auxiliary problem

$$
\begin{aligned}
\rho \omega \bar{u}-\mu_{*} \Delta \bar{u}+\nabla \bar{\pi} & =0, & & \text { in } \Omega \backslash \Sigma, \\
\operatorname{div} \bar{u} & =G_{d}(u, h), & & \text { in } \Omega \backslash \Sigma, \\
\rho \kappa_{*} \omega \bar{\vartheta}-d_{*} \Delta \bar{\vartheta} & =0, & & \text { in } \Omega \backslash \Sigma, \\
\bar{u}=\partial_{\nu} \bar{\vartheta} & =0, & & \text { on } \partial \Omega, \\
P_{\Sigma} \llbracket \bar{u} \rrbracket & =G_{u}(u, \vartheta, h), & & \text { on } \Sigma, \\
-P_{\Sigma} \llbracket \mu_{*}\left(\nabla \bar{u}+\left[\nabla \bar{u} \rrbracket^{\top}\right) \rrbracket \nu_{\Sigma}\right. & =G_{\tau}(u, \vartheta, h), & & \text { on } \Sigma, \\
\llbracket \bar{\vartheta} \rrbracket & =0 & & \text { on } \Sigma \\
-\left(l_{*} / \theta_{*}\right) \llbracket u \cdot \nu_{\Sigma} \rrbracket / \llbracket 1 / \rho \rrbracket-\llbracket d_{*} \partial_{\nu_{\Sigma}} \bar{\vartheta} \rrbracket & =G_{\theta}(u, \vartheta, h), & & \text { on } \Sigma, \\
-\llbracket T(\bar{u}, \bar{\pi}, \bar{\vartheta}) \nu_{\Sigma} \cdot \nu_{\Sigma} \rrbracket & =-\sigma \mathcal{A}_{\Sigma} h, & & \text { on } \Sigma, \\
l_{*} \vartheta-\llbracket T(\bar{u}, \bar{\pi}, \bar{\vartheta}) \nu_{\Sigma} \cdot \nu_{\Sigma} / \rho \rrbracket & =0, & & \text { on } \Sigma,
\end{aligned}
$$

by means of the implicit function theorem, employing Proposition 5.1. Then with $\bar{z}=(\bar{u}, \bar{\vartheta}, 0)$ and $\tilde{z}=(\tilde{u}, \tilde{\vartheta}, \tilde{h}), z=\tilde{z}+\bar{z}$, we obtain $\bar{z}=\phi(\tilde{z})$, with a $C^{1}$-function $\phi$ such that $\phi(0)=\phi^{\prime}(0)=0$. Then $z=\tilde{z}+\phi(\tilde{z}) \in \mathcal{S} \mathcal{M}$, hence $\mathcal{S M}$ is locally near 0 parameterized over $\mathcal{S} \mathcal{X}$. To prove surjectivity of this map, given $(u, \vartheta, h) \in \mathcal{S} \mathcal{M}$ sufficiently small, solve the linear problem

$$
\begin{aligned}
\rho \omega \bar{u}-\mu_{*} \Delta \bar{u}+\nabla \bar{\pi} & =0, & & \text { in } \Omega \backslash \Sigma, \\
\operatorname{div} \bar{u} & =G_{d}(u, h), & & \text { in } \Omega \backslash \Sigma, \\
\rho \kappa_{*} \omega \bar{\vartheta}-d_{*} \Delta \bar{\vartheta} & =0, & & \text { in } \Omega \backslash \Sigma, \\
\bar{u}=\partial_{\nu} \bar{\vartheta} & =0, & & \text { on } \partial \Omega, \\
P_{\Sigma} \llbracket \bar{u} \rrbracket & =G_{u}(u, \vartheta, h), & & \text { on } \Sigma, \\
-P_{\Sigma} \llbracket \mu_{*}\left(\nabla \bar{u}+[\nabla \bar{u}]^{\top}\right) \rrbracket \nu_{\Sigma} & =G_{\tau}(u, \vartheta, h), & & \text { on } \Sigma, \\
\llbracket \bar{\vartheta} \rrbracket & =0 & & \text { on } \Sigma \\
-\left(l_{*} / \theta_{*}\right) \llbracket u \cdot \nu_{\Sigma} \rrbracket / \llbracket 1 / \rho \rrbracket-\llbracket d_{*} \partial_{\nu_{\Sigma}} \bar{\vartheta} \rrbracket & =G_{\theta}(u, \vartheta, h), & & \text { on } \Sigma, \\
-\llbracket T(\bar{u}, \bar{\pi}, \bar{\vartheta}) \nu_{\Sigma} \cdot \nu_{\Sigma} \rrbracket & =-\sigma \mathcal{A}_{\Sigma} h, & & \text { on } \Sigma, \\
l_{*} \vartheta-\llbracket T(\bar{u}, \bar{\pi}, \bar{\vartheta}) \nu_{\Sigma} \cdot \nu_{\Sigma} / \rho \rrbracket & =0, & & \text { on } \Sigma,
\end{aligned}
$$

and set $\tilde{z}=z-\bar{z}$. Then we see that $\bar{z}=\phi(\tilde{z})$, hence the map $\tilde{z} \mapsto \tilde{z}+\phi(\tilde{z})$ is also surjective near 0 .

4. Next, let $w_{\infty}:=\left(z_{\infty}, \pi_{\infty}\right):=\left(u_{\infty}, \vartheta_{\infty}, h_{\infty}, \pi_{\infty}\right)$ be an equilibrium of (5.1) (5.3) such that $z_{\infty}=\tilde{z}_{\infty}+\phi\left(\tilde{z}_{\infty}\right) \in \mathcal{S} \mathcal{M}$ is close to the part $z_{*}=(0,0,0)$ of the fixed equilibrium $w_{*}=\left(0,0,0, \pi_{*}\right)$. Clearly, $u_{\infty}=0, \vartheta_{\infty}=$ const., $\pi_{*}, \pi_{\infty}=$ const. and it holds that $\mathbb{L} w_{\infty}=N\left(w_{\infty}\right)$. 
Given a solution $w \in \mathbb{E}$ of Problem (5.1)-(5.3), we decompose $w$ as $w=\tilde{w}+$ $\bar{w}+w_{\infty}$, where now $\bar{w}$ solves the following auxiliary problem

$$
\begin{aligned}
& \omega \rho \bar{u}+\rho \partial_{t} \bar{u}-\mu_{*} \Delta \bar{u}+\nabla \bar{\pi}=\rho F_{u}(u, \vartheta, h, \pi), \quad \text { in } \Omega \backslash \Sigma, \\
& \operatorname{div} \bar{u}=G_{d}(u, h), \quad \text { in } \Omega \backslash \Sigma, \\
& \rho \kappa_{*} \omega \bar{\vartheta}+\rho \kappa_{*} \partial_{t} \bar{\vartheta}-d_{*} \Delta \bar{\vartheta}=F_{\theta}(u, \vartheta, h), \quad \text { in } \Omega \backslash \Sigma, \\
& \bar{u}=\partial_{\nu} \bar{\vartheta}=0, \quad \text { on } \partial \Omega, \\
& P_{\Sigma} \llbracket \bar{u} \rrbracket=G_{u}(u, \vartheta, h), \quad \text { on } \Sigma, \\
& -P_{\Sigma} \llbracket \mu_{*}\left(\nabla \bar{u}+[\nabla \bar{u}]^{\top}\right) \rrbracket \nu_{\Sigma}=G_{\tau}(u, \vartheta, h), \quad \text { on } \Sigma, \\
& \llbracket \bar{\vartheta} \rrbracket=0 \quad \text { on } \Sigma \\
& -\left(l_{*} / \theta_{*}\right) \llbracket \bar{u} \cdot \nu_{\Sigma} \rrbracket / \llbracket 1 / \rho \rrbracket-\llbracket d_{*} \partial_{\nu_{\Sigma}} \bar{\vartheta} \rrbracket=G_{\theta}(u, \vartheta, h), \quad \text { on } \Sigma, \\
& -\llbracket T(\bar{u}, \bar{\pi}, \bar{\vartheta}) \nu_{\Sigma} \cdot \nu_{\Sigma} \rrbracket+\sigma \mathcal{A}_{\Sigma} \bar{h}=G_{\nu}(u, \vartheta, h)+G_{\gamma}(h)-G_{\gamma}\left(h_{\infty}\right), \quad \text { on } \Sigma, \\
& l_{*} \vartheta-\llbracket T(\bar{u}, \bar{\pi}, \bar{\vartheta}) \nu_{\Sigma} \cdot \nu_{\Sigma} / \rho \rrbracket=G_{h}(u, \vartheta, h), \quad \text { on } \Sigma, \\
& \omega \bar{h}+\partial_{t} \bar{h}-\llbracket \rho \bar{u} \cdot \nu_{\Sigma} \rrbracket / \llbracket \rho \rrbracket=F_{h}(u, h), \quad \text { on } \Sigma, \\
& \bar{u}(0)=\bar{u}_{0}, \quad \bar{\vartheta}(0)=\bar{\vartheta}_{0}, \quad \bar{h}(0)=\bar{h}_{0},
\end{aligned}
$$

where $z_{0}=\tilde{z}_{0}+\bar{z}_{0}=\tilde{z}_{0}+\phi\left(\tilde{z}_{0}\right)$. The remaining problem for $\tilde{w}$ reads

$$
\begin{array}{rlrl}
\rho \partial_{t} \tilde{u}-\mu_{*} \Delta \tilde{u}+\nabla \tilde{\pi} & =\omega \rho \bar{u}, & & \text { in } \Omega \backslash \Sigma, \\
\operatorname{div} \tilde{u} & =0, & & \text { in } \Omega \backslash \Sigma, \\
\rho \kappa_{*} \partial_{t} \tilde{\vartheta}-d_{*} \Delta \tilde{\vartheta} & =\rho \kappa_{*} \omega \bar{\vartheta}, & & \text { in } \Omega \backslash \Sigma, \\
\tilde{u}=\partial_{\nu} \tilde{\vartheta} & =0, & & \text { on } \partial \Omega, \\
P_{\Sigma} \llbracket \tilde{u} \rrbracket=0,-P_{\Sigma} \llbracket \mu_{*}\left(\nabla \tilde{u}+\left[\nabla \tilde{u} \rrbracket^{\top}\right) \rrbracket \nu_{\Sigma}\right. & =0, & & \text { on } \Sigma, \\
\llbracket \tilde{\vartheta} \rrbracket & =0 & & \text { on } \Sigma \\
\left(l_{*} / \theta_{*}\right) \llbracket \tilde{u} \cdot \nu_{\Sigma} \rrbracket / \llbracket 1 / \rho \rrbracket-\llbracket d_{*} \partial_{\nu_{\Sigma}} \tilde{\vartheta} \rrbracket & =0, \\
-\llbracket T(\tilde{u}, \tilde{\pi}, \tilde{\vartheta}) \nu_{\Sigma} \cdot \nu_{\Sigma} \rrbracket+\sigma \mathcal{A}_{\Sigma} \tilde{h} & =0, & & \text { on } \Sigma \\
l_{*} \tilde{\vartheta}-\llbracket T(\tilde{u}, \tilde{\pi}, \tilde{\vartheta}) \nu_{\Sigma} \cdot \nu_{\Sigma} / \rho \rrbracket & =0, & & \text { on } \Sigma, \\
\partial_{t} \tilde{h}-\llbracket \rho \tilde{u} \cdot \nu_{\Sigma} \rrbracket / \llbracket \rho \rrbracket & =\omega \bar{h}, & & \text { on } \Sigma, \\
\tilde{u}(0)=\tilde{u}_{0}, \quad \tilde{\vartheta}(0)=\tilde{\vartheta}_{0}, \quad \tilde{h}(0) & =\tilde{h}_{0} . & & \text { on } \Sigma,
\end{array}
$$

5. Problem (5.7) can be written abstractly as

$$
\mathbb{L}_{\omega} \bar{w}=N\left(\bar{w}+\tilde{w}+w_{\infty}\right)-N\left(w_{\infty}\right) .
$$

And (5.8) abstractly becomes the evolution equation

$$
\dot{\tilde{z}}+L \tilde{z}=R(\bar{z}), \quad t>0, \tilde{z}(0)=\tilde{z}_{0}
$$


in the Banach space $X_{0}$, with $L$ defined as in Section 4 and $R(\bar{z}):=\omega((I-$ $\left.\left.T_{1}\right) \bar{u}, \kappa_{*} \bar{\vartheta}, \bar{h}\right)$. This abstract problem can be treated in the same way as in the proof of Theorem 5.2 in Prüss, Simonett and Zacher [26. This implies the following result.

Theorem 5.2. Let $p>n+2, \sigma, \rho_{1}, \rho_{2}>0, \rho_{1} \neq \rho_{2}$, and suppose $\psi_{j} \in C^{3}(0, \infty)$, $\mu_{j}, d_{j} \in C^{2}(0, \infty)$ are such that

$$
\kappa_{j}(s)=-s \psi_{j}^{\prime \prime}(s)>0, \quad \mu_{j}(s)>0, \quad d_{j}(s)>0, \quad s \in(0, \infty), j=1,2 .
$$

Then in the topology of the state manifold $\mathcal{S} \mathcal{M}$ we have:

(i) $\left(0, \theta_{*}, \Gamma_{*}\right) \in \mathcal{E}$ is stable if and only if $\Gamma_{*}$ is connected.

(ii) Any solution starting in a neighborhood of a stable equilibrium exists globally and converges to a probably different stable equilibrium in the topology of $\mathcal{S} \mathcal{M}$.

(iii) Any solution starting and staying in a neighborhood of an unstable equilibrium exists globally and converges to a probably different unstable equilibrium in the topology of $\mathcal{S} \mathcal{M}$.

\section{Global Existence and Convergence}

In this section we study the global properties of problem (1.1), (1.2), (1.3)

6.1. The Local Semiflow. We follow here the approach introduced in Köhne, Prüss and Wilke 14] for the isothermal incompressible two-phase Navier-Stokes problem without phase transitions and in Prüss, Simonett and Zacher 26] for the Stefan problem with surface tension.

Recall that the closed $C^{2}$-hyper-surfaces contained in $\Omega$ form a $C^{2}$-manifold, which we denote by $\mathcal{M H}^{2}(\Omega)$. The charts are the parameterizations over a given hyper-surface $\Sigma$ according to Section 2, and the tangent space consists of the normal vector fields on $\Sigma$. We define a metric on $\mathcal{M H}^{2}(\Omega)$ by means of

$$
d_{\mathcal{M H}^{2}}\left(\Sigma_{1}, \Sigma_{2}\right):=d_{H}\left(\mathcal{N}^{2} \Sigma_{1}, \mathcal{N}^{2} \Sigma_{2}\right),
$$

where $d_{H}$ denotes the Haussdorff metric on the compact subsets of $\mathbb{R}^{n}$ introduced in Section 2. This way $\mathcal{M H}^{2}(\Omega)$ becomes a Banach manifold of class $C^{2}$.

Let $d_{\Sigma}(x)$ denote the signed distance for $\Sigma$. We may then define the level function $\varphi_{\Sigma}$ by means of

$$
\varphi_{\Sigma}(x)=\phi\left(d_{\Sigma}(x)\right), \quad x \in \mathbb{R}^{n}
$$

where

$$
\phi(s)=s(1-\chi(s / a))+\operatorname{sgn} s \chi(s / a), \quad s \in \mathbb{R} .
$$

It is easy to see that $\Sigma=\varphi_{\Sigma}^{-1}(0)$, and $\nabla \varphi_{\Sigma}(x)=\nu_{\Sigma}(x)$, for each $x \in \Sigma$. Moreover, $\kappa=0$ is an eigenvalue of $\nabla^{2} \varphi_{\Sigma}(x)$, the remaining eigenvalues of $\nabla^{2} \varphi_{\Sigma}(x)$ are the principal curvatures $\kappa_{j}$ of $\Sigma$ at $x \in \Sigma$.

If we consider the subset $\mathcal{M H}^{2}(\Omega, r)$ of $\mathcal{M H}^{2}(\Omega)$ which consists of all closed hyper-surfaces $\Gamma \in \mathcal{M H}^{2}(\Omega)$ such that $\Gamma \subset \Omega$ satisfies the ball condition with 
fixed radius $r>0$ then the map $\Phi: \mathcal{M H}^{2}(\Omega, r) \rightarrow C^{2}(\bar{\Omega})$ defined by $\Phi(\Gamma)=\varphi_{\Gamma}$ is an isomorphism of the metric space $\mathcal{M H}^{2}(\Omega, r)$ onto $\Phi\left(\mathcal{M H}^{2}(\Omega, r)\right) \subset C^{2}(\bar{\Omega})$.

Let $s-(n-1) / p>2$; for $\Gamma \in \mathcal{M H}^{2}(\Omega, r)$, we define $\Gamma \in W_{p}^{s}(\Omega, r)$ if $\varphi_{\Gamma} \in W_{p}^{s}(\Omega)$. In this case the local charts for $\Gamma$ can be chosen of class $W_{p}^{s}$ as well. A subset $A \subset W_{p}^{s}(\Omega, r)$ is (relatively) compact, if and only if $\Phi(A) \subset W_{p}^{s}(\Omega)$ is (relatively) compact.

As an ambient space for the state manifold $\mathcal{S M}$ of Problem (1.1), (1.2), (1.3) we consider the product space $L_{p}(\Omega)^{n+1} \times \mathcal{M H}^{2}$.

We define the state manifold for the problem $\mathcal{S M}$ as follows.

$$
\begin{gathered}
\mathcal{S M}:=\left\{(u, \theta, \Gamma) \in L_{p}(\Omega)^{n+1} \times \mathcal{M H}^{2}(\Omega):(u, \theta) \in W_{p}^{2-2 / p}(\Omega \backslash \Gamma)^{n+1}, \Gamma \in W_{p}^{3-2 / p},\right. \\
\operatorname{div} u=0 \text { in } \Omega, \quad \theta>0 \text { in } \bar{\Omega}, \quad u=\partial_{\nu} \theta=0 \text { on } \partial \Omega, \\
\llbracket \theta \rrbracket=\llbracket P_{\Gamma} u \rrbracket=P_{\Gamma} \llbracket \mu(\theta)\left(\nabla u+[\nabla u]^{\top}\right) \rrbracket \nu_{\Gamma}=0 \quad \text { on } \Gamma, \\
\left.l(\theta) \llbracket u \cdot \nu_{\Gamma} \rrbracket / \llbracket 1 / \rho \rrbracket+\llbracket d(\theta) \partial_{\nu_{\Gamma}} \theta \rrbracket=0 \quad \text { on } \Gamma\right\},
\end{gathered}
$$

Charts for these manifolds are obtained by the charts induced by $\mathcal{M H}^{2}(\Omega)$, followed by a Hanzawa transformation.

Applying Theorem 3.2 and re-parameterizing the interface repeatedly, we see that (1.1), (1.2), (1.3) yields a local semiflow on $\mathcal{S M}$.

Theorem 6.1. Let $p>n+2, \sigma, \rho_{1}, \rho_{2}>0, \rho_{1} \neq \rho_{2}$, and suppose $\psi_{j} \in C^{3}(0, \infty)$, $\mu_{j}, d_{j} \in C^{2}(0, \infty)$ such that

$$
\kappa_{j}(s)=-s \psi_{j}^{\prime \prime}(s)>0, \quad \mu_{j}(s)>0, \quad d_{j}(s)>0, \quad s \in(0, \infty), j=1,2 .
$$

Then problem (1.1), (1.2), (1.3) generates a local semiflow on the state manifold $\mathcal{S M}$. Each solution $(u, \theta, \Gamma)$ of the problem exists on a maximal time interval $\left[0, t_{*}\right)$, where $t_{*}=t_{*}\left(u_{0}, \theta_{0}, \Gamma_{0}\right)$.

Note that the pressure $\pi$ as well as the phase flux $j$ are dummy variables which are determined for each $t$ by the principal variables $(u, \theta, \Gamma)$. In fact, $j$ is given by

$$
j=\llbracket u \cdot \nu_{\Gamma} \rrbracket / \llbracket 1 / \rho \rrbracket,
$$

and $\pi$ is determined by the weak transmission problem

$$
\begin{aligned}
& (\nabla \pi \mid \nabla \phi / \rho)_{L_{2}(\Omega)}=\left(2 \rho^{-1} \operatorname{div}(\mu(\theta) D(u))-u \cdot \nabla u \mid \nabla \phi\right)_{L_{2}(\Omega)}, \quad \phi \in H_{p^{\prime}}^{1}(\Omega), \\
& \llbracket \pi \rrbracket=2 \llbracket \mu(\theta) D(u) \nu_{\Gamma} \cdot \nu_{\Gamma} \rrbracket+\sigma H_{\Gamma}-\llbracket 1 / \rho \rrbracket j^{2}, \\
& \llbracket \pi / \rho \rrbracket=2 \llbracket(\mu(\theta) / \rho) D(u) \nu_{\Gamma} \cdot \nu_{\Gamma} \rrbracket-\llbracket 1 / 2 \rho^{2} \rrbracket j^{2}-\llbracket \psi(\theta) \rrbracket \quad \text { on } \Gamma,
\end{aligned}
$$

where $D(u)=\left(\nabla u+[\nabla u]^{\top}\right) / 2$ as before. Concerning such transmission problems we refer to [14. 
6.2. Compactness of Orbits. Let us briefly comment on the fact of how one can use the time weights $t^{1-\mu}, \mu \in(1 / p, 1]$ introduced in Subsection 3.3, to prove relative compactness of bounded orbits in $\mathcal{S M}$. For simplicity we assume that the free boundary is fixed, hence we do only consider the orbits $\{u(t)\}_{t \in\left[0, t_{*}\right)}$ and $\{\theta(t)\}_{t \in\left[0, t_{*}\right)}$ of the velocity field $u$ and the temperature field $\theta$, respectively.

Assume that there exists $M>0$ such that $|u(t)|_{W_{p}^{2-2 / p}}+|\theta(t)|_{W_{p}^{2-2 / p}} \leq M$ for all $t \in\left[0, t_{*}\right)$. Since $\Omega$ is bounded it follows that $\{u(t)\}_{t \in\left[0, t_{*}\right)}$ and $\{\theta(t)\}_{t \in\left[0, t_{*}\right)}$ are relatively compact w.r.t. the topology of $W_{p}^{2 \mu-2 / p}$. The continuous dependence of the solution on the initial data in $W_{p}^{2 \mu-2 / p}$ and the instantaneous regularization of the solution, see Corollary 3.3, yield that the orbits of $u$ and $\theta$ are also relatively compact in $W_{p}^{2-2 / p}$. This in turn yields that the solution exists globally, i.e. $t_{*}=\infty$. We refer to 13 for more details.

If one considers free boundary problems, then one has to work in the setting which has been introduced in Subsection 6.1 In particular, to show relative compactness of the orbits in $\mathcal{S M}$ one has to perform several Hanzawa transforms for each of the finitely many balls which cover the relatively compact set $\{\Gamma(t)\}_{t \geq 0}$ in $W_{p}^{3-2 / p-\varepsilon}(\Omega, r)$. The pull backs of the velocity and temperature field for each of these balls will then be relatively compact in $W_{p}^{2-2 / p-\varepsilon}$. More details are given in the proof of Theorem 6.2 below.

6.3. Convergence. There are several obstructions against global existence:

- regularity: the norms of either $u(t), \theta(t)$, or $\Gamma(t)$ become unbounded;

- geometry: the topology of the interface changes; or the interface touches the boundary of $\Omega$.

- well-posedness: the temperature becomes 0 .

Note that the compatibility conditions,

$$
\begin{aligned}
& \operatorname{div} u(t)=0 \text { in } \Omega \backslash \Gamma(t), \quad u=\partial_{\nu} \theta=0 \text { on } \partial \Omega, \\
& P_{\Gamma} \llbracket u(t) \rrbracket=\llbracket \theta \rrbracket=P_{\Gamma} \llbracket \mu D(u)(t) \rrbracket=l(\theta) \llbracket u \cdot \nu_{\Gamma} / \rrbracket \llbracket 1 / \rho \rrbracket+\llbracket d(\theta) \partial_{\nu_{\Gamma}} \theta \rrbracket=0 \text { on } \Gamma(t),
\end{aligned}
$$

are preserved by the semiflow.

Let $(u, \theta, \Gamma)$ be a solution in the state manifold $\mathcal{S M}$ with maximal interval $\left[0, t_{*}\right)$. By the uniform ball condition we mean the existence of a radius $r_{0}>0$ such that for each $t$, at each point $x \in \Gamma(t)$ there exists centers $x_{i} \in \Omega_{i}(t)$ such that $B_{r_{0}}\left(x_{i}\right) \subset \Omega_{i}$ and $\Gamma(t) \cap \bar{B}_{r_{0}}\left(x_{i}\right)=\{x\}, i=1,2$. Note that this condition bounds the curvature of $\Gamma(t)$, prevents parts of it to touch the outer boundary $\partial \Omega$, and to undergo topological changes. Hence if this condition holds, then the volumes of the phases are preserved.

With this property, combining the local semiflow for (1.1), (1.2), (1.3) with the Ljapunov functional and compactness we obtain the following result. 
Theorem 6.2. Let $p>n+2, \sigma, \rho_{1}, \rho_{2}>0, \rho_{1} \neq \rho_{2}$, and suppose $\psi_{j} \in C^{3}(0, \infty)$, $\mu_{j}, d_{j} \in C^{2}(0, \infty)$ are such that

$$
\kappa_{j}(s)=-s \psi_{j}^{\prime \prime}(s)>0, \quad \mu_{j}(s)>0, \quad d_{j}(s)>0, \quad s \in(0, \infty), j=1,2 .
$$

Suppose that $(u, \theta, \Gamma)$ is a solution of (1.1), (1.2), (1.3) in the state manifold $\mathcal{S M}$ on its maximal time interval $\left[0, t_{*}\right)$. Assume there is constant $M>0$ such that the following conditions hold on $\left[0, t_{*}\right)$ :

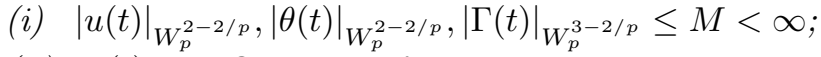

(ii) $\Gamma(t)$ satisfies the uniform ball condition.

(iii) $\theta(t) \geq 1 / M$ on $\bar{\Omega}$.

Then $t_{*}=\infty$, i.e. the solution exists globally, and its limit set $\omega_{+}(u, \theta, \Gamma) \subset \mathcal{E}$ is non-empty. If further $\left(0, \theta_{\infty}, \Gamma_{\infty}\right) \in \omega_{+}(u, \theta, \Gamma)$ with $\Gamma_{\infty}$ connected, then the solution converges in $\mathcal{S M}$ to this equilibrium.

Conversely, if $(u(t), \theta(t), \Gamma(t))$ is a global solution in $\mathcal{S M}$ which converges to an equilibrium $\left(0, \theta_{*}, \Gamma_{*}\right) \in \mathcal{E}$ in $\mathcal{S} \mathcal{M}$ as $t \rightarrow \infty$, then (i), (ii) and (iii) are valid.

Proof. Assume that (i), (ii) and (iii) are valid. Then $\Gamma\left(\left[0, t_{*}\right)\right) \subset W_{p}^{3-2 / p}(\Omega, r)$ is bounded, hence relatively compact in $W_{p}^{3-2 / p-\varepsilon}(\Omega, r)$. Thus we may cover this set by finitely many balls with centers $\Sigma_{k}$ real analytic in such a way that

$$
\operatorname{dist}_{W_{p}^{3-2 / p-\varepsilon}}\left(\Gamma(t), \Sigma_{j}\right) \leq \delta
$$

for some $j=j(t), t \in\left[0, t_{*}\right)$. Let $J_{k}=\left\{t \in\left[0, t_{*}\right): j(t)=k\right\}$; using for each $k$ a Hanzawa-transformation $\Xi_{k}$, we see that the pull backs $\left\{(u(t, \cdot), \theta(t, \cdot)) \circ \Xi_{k}: t \in\right.$ $\left.J_{k}\right\}$ are bounded in $W_{p}^{2-2 / p}\left(\Omega \backslash \Sigma_{k}\right)^{n+1}$, hence relatively compact in $W_{p}^{2-2 / p-\varepsilon}(\Omega \backslash$ $\left.\Sigma_{k}\right)^{n+1}$. Employing now Corollary 3.3 we obtain solutions $\left(u^{1}, \theta^{1}, \Gamma^{1}\right)$ with initial configurations $(u(t), \theta(t), \Gamma(t))$ in the state manifold on a common time interval say $(0, \tau]$, and by uniqueness we have

$$
\left(u^{1}(\tau), \theta^{1}(\tau), \Gamma^{1}(\tau)\right)=(u(t+\tau), \theta(t+\tau), \Gamma(t+\tau)) .
$$

Continuous dependence implies that the orbit of the solution $(u(\cdot), \theta(\cdot), \Gamma(\cdot))$ is relative compact in $\mathcal{S M}$, in particular $t_{*}=\infty$ and $(u, \theta, \Gamma)\left(\mathbb{R}_{+}\right) \subset \mathcal{S M}$ is relatively compact. The negative total entropy is a strict Ljapunov functional, hence the limit set $\omega_{+}(u, \theta, \Gamma) \subset \mathcal{S M}$ of a solution is contained in the set $\mathcal{E}$ of equilibria. By compactness $\omega_{+}(u, \theta, \Gamma) \subset \mathcal{S} \mathcal{M}$ is non-empty, hence the solution comes close to $\mathcal{E}$, and stays there. Then we may apply the convergence result Theorem5.2. The converse statement follows by a compactness argument.

\section{Proof of Theorems 3.1}

In this section, we prove Theorem 3.1] employing the methods introduced in [14. Actually, most of the arguments given in that paper remain valid for the problem under consideration here, hence we restrict on the necessary modifications. 
7.1. Flat interface. In this subsection, we consider first the linear problem with constant coefficients for a flat interface. Due to the jump in the velocity, this problem differs from that in [14.

$$
\begin{aligned}
\rho \partial_{t} u-\mu_{0} \Delta u+\nabla \pi & =\rho f_{u} & & \text { in } \dot{\mathbb{R}}^{n}, \\
\operatorname{div} u & =g_{d} & & \text { in } \dot{\mathbb{R}}^{n}, \\
\llbracket v \rrbracket+c_{0} \nabla_{x} h & =P_{\Sigma} g_{u} & & \text { on } \mathbb{R}^{n-1}, \\
-\llbracket \mu_{0} \partial_{y} v \rrbracket-\llbracket \mu_{0} \nabla_{x} w \rrbracket & =g_{v} & & \text { on } \mathbb{R}^{n-1}, \\
-2 \llbracket \mu_{0} \partial_{y} w \rrbracket+\llbracket \pi \rrbracket-\sigma \Delta_{x} h & =g_{w} & & \text { on } \mathbb{R}^{n-1}, \\
-2 \llbracket \mu_{0} \partial_{y} w / \rho \rrbracket+\llbracket \pi / \rho \rrbracket & =g_{h} & & \text { on } \mathbb{R}^{n-1}, \\
\partial_{t} h-\llbracket \rho w \rrbracket / \llbracket \rho \rrbracket+b_{0} \cdot \nabla_{x} h / \llbracket \rho \rrbracket & =f_{h} & & \text { on } \mathbb{R}^{n-1}, \\
u(0) & =u_{0} & & \text { in } \dot{\mathbb{R}}^{n}, \\
h(0) & =h_{0} & & \text { on } \mathbb{R}^{n-1},
\end{aligned}
$$

with $\mu_{0}>0, c_{0} \in \mathbb{R}, b_{0} \in \mathbb{R}^{n-1}$. Here we have identified $\mathbb{R}^{n-1}=\mathbb{R}^{n-1} \times\{0\}$, and $\dot{\mathbb{R}}^{n}=\mathbb{R}^{n} \backslash \mathbb{R}^{n-1}$. It is convenient to split $u=(v, w), g=\left(g_{v}, g_{w}\right)$ into tangential and normal components. Since the 3rd and the 7th equation differ from the coupled system (3.1) and (3.3) in 23], we solve (7.1) with all right hand sides 0 except $f_{h}$. Employing Laplace transform in $t$ and Fourier transform in the tangential variable $x \in \mathbb{R}^{n-1}$ similar to Sec.4 of [23], the transformed equation for $\hat{h}$ reads

$$
\lambda \hat{h}-\frac{\llbracket \rho \hat{w}(0) \rrbracket}{\llbracket \rho \rrbracket}+\frac{b_{0} \cdot i \xi \hat{h}}{\llbracket \rho \rrbracket}=\hat{f}_{h} .
$$

We obtain after some linear algebra

$$
s(\lambda,|\xi|) \hat{h}=\hat{f}_{h} .
$$

Setting $\tau=|\xi|$ and employing the scaling $z=\lambda / \tau^{2}$, we obtain the boundary symbol $s(\lambda, \tau)$ in the form

$$
s(\lambda, \tau)=\lambda+\frac{\sigma \tau}{\llbracket \rho \rrbracket^{2}} m(z)+\frac{c_{0} \tau}{\llbracket \rho \rrbracket} \ell(z)+\frac{i \tau}{\llbracket \rho \rrbracket}\left(b_{0} \cdot \frac{\xi}{|\xi|}\right) .
$$

We derive this formula in the Appendix. The first and the second terms are the same symbols as in the system (3.1) and (3.3) in [23. The holomorphic function $m(z)$ is the same as (4.14) in [23] and $\ell(z)$ is given by

$$
\begin{aligned}
\psi(z) \ell(z)=\rho_{1} \mu_{2} \frac{\omega_{1}(z)-1}{\omega_{1}(z)\left(\omega_{1}(z)^{2}+1\right)}\left(\frac{\omega_{2}(z)-1}{\omega_{2}(z)}+2 \frac{\omega_{2}(z)+1}{\omega_{2}(z)^{2}+1}\right) \\
-\rho_{2} \mu_{1} \frac{\omega_{2}(z)-1}{\omega_{2}(z)\left(\omega_{2}(z)^{2}+1\right)}\left(\frac{\omega_{1}(z)-1}{\omega_{1}(z)}+2 \frac{\omega_{1}(z)+1}{\omega_{1}(z)^{2}+1}\right),
\end{aligned}
$$


where $\omega_{j}(z)=\sqrt{1+\rho_{j} z / \mu_{j}}$ and

$$
\begin{aligned}
\psi(z)=\frac{\omega_{1}(z)+1}{\omega_{1}(z)^{2}+1} \mu_{2}\left(\frac{\omega_{2}(z)-1}{\omega_{2}(z)}\right. & \left.+2 \frac{\omega_{2}(z)+1}{\omega_{2}(z)^{2}+1}\right) \\
& +\frac{\omega_{2}(z)+1}{\omega_{2}(z)^{2}+1} \mu_{1}\left(\frac{\omega_{1}(z)-1}{\omega_{1}(z)}+2 \frac{\omega_{1}(z)+1}{\omega_{1}(z)^{2}+1}\right) .
\end{aligned}
$$

Note that $\omega_{j}(z)$ is holomorphic in the sliced plane $\mathbb{C} \backslash\left(-\infty,-\frac{\mu_{j}}{\rho_{j}}\right]$, hence the function $\psi(z)$ has this property in $\mathbb{C} \backslash(-\infty, \eta]$, with $\eta=\min \left\{\mu_{j} / \rho_{j}\right\}$. It is not difficult to see that $\psi$ maps $\overline{\mathbb{C}}_{+}$into $\mathbb{C}_{+}$, and with $\psi(0)=2\left(\mu_{1}+\mu_{2}\right), \psi(z) \rightarrow$ $\frac{\mu_{1}}{\sqrt{z}} \sqrt{\frac{\mu_{2}}{\rho_{2}}}+\frac{\mu_{2}}{\sqrt{z}} \sqrt{\frac{\mu_{1}}{\rho_{1}}}$ as $|z| \rightarrow \infty$ we may conclude $\psi\left(\overline{\mathbb{C}}_{+}\right) \subset \Sigma_{\phi}$ for some angle $\phi<\frac{\pi}{2}$. By continuity of the argument function, this implies $\psi\left(\Sigma_{\frac{\pi}{2}+\eta}\right) \subset \mathbb{C}_{+}$ for some $\eta>0$. Therefore $\psi(z)$ cannot vanish in $\Sigma_{\frac{\pi}{2}+\eta}$. This implies that $\ell(z)$ is holomorphic in this sector and in a ball $B_{r_{0}}(0)$ for some $r_{0}>0$. For the asymptotic of $\ell(z)$ we have

$$
\ell(0)=0, \quad \lim _{|z| \rightarrow \infty} z \ell(z)=\frac{2 \mu_{1} \mu_{2}\left(\sqrt{\mu_{2} / \rho_{2}}-\sqrt{\mu_{1} / \rho_{1}}\right)}{\mu_{1} \sqrt{\mu_{2} / \rho_{2}}+\mu_{2} \sqrt{\mu_{1} / \rho_{1}}} \quad \text { for } z \in \mathbb{C} \backslash \mathbb{R}_{-} .
$$

Thus there is a constant $L=L(r, \phi)>0$ such that

$$
|\ell(z)| \leq \frac{L}{1+|z|} \quad z \in \Sigma_{\phi} \cup B_{r}(0)
$$

for each $\phi<\frac{\pi}{2}+\eta$ and $r<r_{0}$. Combining this estimate with the estimate for $m(z)$ from Sec.4 in [23], it is easy to conclude

$$
|s(\lambda, \tau)| \leq c_{\eta}(|\lambda|+|\tau|) \quad \lambda \in \Sigma_{\frac{\pi}{2}+\eta}, \quad \tau \in \Sigma_{\eta},
$$

whenever $\eta>0$ is small enough. Conversely since $m(0)>0$, given a small $\eta>0$ we find $\tau_{\eta} \in\left(0, r_{0}\right)$ such that $m(z) \in \Sigma_{\frac{\pi}{2}-3 \eta}$ and $|m(z)| \geq \frac{m(0)}{2}$ for all $\tau \in B_{r_{\eta}}(0)$. This implies that there is a constant $c_{\eta}>0$ such that

$$
|s(\lambda, \tau)| \geq|\lambda|+|\tau| \frac{\sigma}{2 \llbracket \rho \rrbracket^{2}} m(0), \quad \lambda \in \Sigma_{\frac{\pi}{2}+\eta}, \quad|\lambda| \leq r_{\eta}|\tau|^{2} .
$$

On the other hand, choosing $|\lambda| \geq C|\tau|$ we obtain

$$
|s(\lambda, \tau)| \geq \frac{|\lambda|}{2}+\left(\frac{C}{2}-\frac{\sigma}{\llbracket \rho \rrbracket^{2}} M-\frac{\left|c_{0}\right|}{\llbracket \rho \rrbracket} L-\frac{\left|b_{0}\right|}{\llbracket \rho \rrbracket}\right)|\tau| \geq c_{\eta}(|\lambda|+|\tau|)
$$

for all $\lambda \in \Sigma_{\frac{\pi}{2}+\eta}, \tau \in \Sigma_{\eta}$, with $|\lambda| \geq C|\tau|$ and

$$
C>2\left(\sigma M / \llbracket \rho \rrbracket^{2}+\left|c_{0}\right| L / \llbracket \rho \rrbracket+\left|b_{0}\right| / \llbracket \rho \rrbracket\right) .
$$

Therefore if $\lambda_{0}$ is chosen large enough this implies the lower bound

$$
|s(\lambda, \tau)| \geq c_{\eta}(|\lambda|+|\tau|) \quad \lambda \in \Sigma_{\frac{\pi}{2}+\eta}, \tau \in \Sigma_{\eta},|\lambda| \geq \lambda_{0} .
$$

Thus this boundary symbol has the same regularity behavior for $|\lambda| \geq \lambda_{0}$ as that for the problem in 23 . 
The linear problem with variable coefficients but small deviations for a flat interface, i.e.

$$
\begin{aligned}
& \rho \partial_{t} u-\mu(x) \Delta u+\nabla \pi=\rho f_{u} \quad \text { in } \dot{\mathbb{R}}^{n}, \quad t>0, \\
& \operatorname{div} u=g_{d} \quad \text { in } \dot{\mathbb{R}}^{n}, \quad t>0, \\
& \llbracket v \rrbracket+c(t, x) \nabla_{x} h=P_{\Sigma} g_{u} \quad \text { on } \mathbb{R}^{n-1}, \quad t>0, \\
& -\llbracket \mu(x) \partial_{y} v \rrbracket-\llbracket \mu_{0} \nabla_{x} w \rrbracket=g_{v} \quad \text { on } \mathbb{R}^{n-1}, \quad t>0, \\
& -2 \llbracket \mu(x) \partial_{y} w \rrbracket+\llbracket \pi \rrbracket-\sigma \Delta_{x} h=g_{w} \quad \text { on } \mathbb{R}^{n-1}, \quad t>0, \\
& -2 \llbracket \mu_{0} \partial_{y} w / \rho \rrbracket+\llbracket \pi / \rho \rrbracket=g_{h} \quad \text { on } \mathbb{R}^{n-1}, \quad t>0, \\
& \partial_{t} h-\llbracket \rho w \rrbracket / \llbracket \rho \rrbracket+b(t, x) \cdot \nabla_{x} h / \llbracket \rho \rrbracket=f_{h} \quad \text { on } \mathbb{R}^{n-1}, \quad t>0, \\
& u(0)=u_{0} \quad \text { in } \dot{\mathbb{R}}^{n}, \\
& h(0)=h_{0} \quad \text { on } \mathbb{R}^{n-1} \text {, }
\end{aligned}
$$

can be handled by a perturbation argument in the same way as in 14. The same is true for bent interfaces. However, the localization argument needs some significant modifications, which we explain in some detail now.

7.2. General Bounded Geometries. Here we use the method of localization. By assumption, $\partial \Omega$ is bounded in class $C^{3-}$ and $\Sigma$ is bounded and real analytic, so in particular of class $C^{4}$. Therefore we cover $\Sigma$ by $N$ balls $B_{r / 2}\left(x_{k}\right)$ with radius $r>0$ and centers $x_{k} \in \Sigma$ such that $\Sigma \cap B_{r}\left(x_{k}\right)$ can be parametrized over the tangent space $T_{x_{k}} \Sigma$ by a function $\theta_{k} \in C^{4}$ such that $\left|\nabla \theta_{k}\right|_{\infty} \leq \eta$ with $\eta>0$ defined as in the previous section. We extend these functions $\theta_{k}$ to all of $T_{x_{k}} \Sigma$ retaining the bound on $\nabla \theta_{k}$. In this way we have created $N$ bent half spaces $\Sigma_{k}$ to which the result proved in the previous subsection applies. We also suppose that $B_{r}\left(x_{k}\right) \subset \Omega$ for each $k$. Set $U:=\Omega \backslash \cup_{j=1}^{N} \overline{B_{r / 2}\left(x_{k}\right)}$ and $U_{k}=B_{r}\left(x_{k}\right)$, $k=1, \ldots, N$. The open set $U$ consists of one component $U_{0}$ characterized by $\partial \Omega \subset \overline{U_{0}}$ and $m$ components open sets say $U_{N+1}, \ldots, N_{N+m}$, which are interior to $\Sigma$, i.e., $\cup_{l=1}^{m} U_{N+l} \subset \Omega_{1}$. Fix a partition of unity $\left\{\varphi_{k}\right\}_{j=0}^{N+m}$ subject to the covering $\left\{U_{k}\right\}_{j=0}^{N+m}$ of $\Omega, \varphi \in C_{0}^{\infty}\left(\mathbb{R}^{n}\right), 0 \leq \varphi_{k} \leq 1$ and $\sum_{j=0}^{N+m} \varphi_{k} \equiv 1$. Note that $\varphi_{0}=1$ in a neighborhood of $\partial \Omega$. Let $\tilde{\varphi}_{k}$ denote cut-off functions with support in $U_{k}$ such that $\tilde{\varphi}_{k}=1$ on the support of $\varphi_{k}$.

Let $z=(u, \pi, \llbracket \pi \rrbracket, h)$ be a solution of (3.4) where we assume without loss of generality $u_{0}=h_{0}=f=g_{d}=0$. We then set $u_{k}=\varphi_{k} u, \pi_{k}=\varphi_{k} \pi, h_{k}=\varphi_{k} h$. 
Then for $k=1, \ldots, N z_{k}=\left(u_{k}, \pi_{k}, \llbracket \pi_{k} \rrbracket, h_{k}\right)$ satisfy the problems

$$
\begin{array}{lr}
\rho \partial_{t} u_{k}-\mu(x) \Delta u_{k}+\nabla \pi_{k}=F_{k}(u, \pi) & \text { in } \mathbb{R}^{n} \backslash \Sigma_{k}, \\
\operatorname{div} u_{k}=\left(\nabla \varphi_{k} \mid u\right) & \text { in } \mathbb{R}^{n} \backslash \Sigma_{k}, \\
P_{\Sigma_{k}} \llbracket u_{k} \rrbracket+c(t, x) \nabla_{\Sigma_{k}} h_{k}=\varphi_{k} P_{\Sigma_{k}} g_{u}+G_{u_{k}}(h) & \text { on } \Sigma_{k}, \\
\llbracket-\mu(x)\left(\nabla u_{k}+\left[\nabla u_{k}\right]^{\top}\right)+\pi_{k} \rrbracket \nu_{\Sigma_{k}}-\sigma\left(\Delta_{\Sigma} h_{k}\right) \nu_{\Sigma_{k}}=\varphi_{k} g_{k}+G_{g_{k}}(u, h) & \text { on } \Sigma_{k}, \\
-\llbracket \mu(x)\left(\nabla u_{k}+\left[\nabla u_{k}\right]^{\top}\right) \nu_{\Sigma_{k}} \cdot \nu_{\Sigma_{k}} / \rho \rrbracket+\llbracket \pi_{k} / \rho \rrbracket=\varphi_{k} g_{h}+G_{h_{k}}(u) & \text { on } \Sigma_{k}, \\
\partial_{t} h_{k}-\llbracket \rho u_{k} \cdot \nu_{\Sigma_{k}} \rrbracket / \llbracket \rho \rrbracket+b(t, x) \cdot \nabla_{\Sigma_{k}} h_{k} / \llbracket \rho \rrbracket=\varphi_{k} f_{h}+F_{h_{k}}(h) & \text { on } \Sigma_{k}, \\
u_{k}(0)=0 & \text { in } \mathbb{R}^{n} \backslash \Sigma_{k}, \\
h_{k}(0)=0 & \text { on } \Sigma_{k},
\end{array}
$$

where

$$
\begin{aligned}
F_{k}(u, \pi) & =\left(\nabla \varphi_{k}\right) \pi-\mu(x)\left[\Delta, \varphi_{k}\right] u, \\
G_{u_{k}}(h) & =c(t, x)\left(\nabla_{\Sigma_{k}} \varphi_{k}\right) h \\
G_{g_{k}}(u, h) & =-\llbracket \mu(x)\left(\nabla_{\Sigma_{k}} \varphi_{k} \otimes u+u \otimes \nabla_{\Sigma_{k}} \varphi_{k}\right) \rrbracket \nu_{\Sigma_{k}}-\sigma\left[\Delta_{\Sigma_{k}}, \varphi_{k}\right] h \nu_{\Sigma_{k}}, \\
G_{h_{k}}(u) & =-\llbracket \mu(x)\left(\nabla_{\Sigma_{k}} \varphi_{k} \otimes u+u \otimes \nabla_{\Sigma_{k}} \varphi_{k}\right) \nu_{\Sigma_{k}} \cdot \nu_{\Sigma_{k}} / \rho \rrbracket, \\
F_{h_{k}}(h) & =\left(b(t, x) \mid\left(\nabla_{\Sigma_{k}} \varphi_{k}\right) h\right) / \llbracket \rho \rrbracket .
\end{aligned}
$$

For $k=0$ we have the standard one-phase Stokes problem with parameters $\rho_{2}$, $\mu_{2}(x)$ on $\Omega$ with no-slip boundary condition on $\partial \Omega$, i.e.,

$$
\begin{aligned}
\rho_{2} \partial_{t} u_{0}-\mu_{2}(x) \Delta u_{0}+\nabla \pi_{0} & =F_{0}(u, \pi) & & \text { in } \Omega \backslash \Sigma, \\
\operatorname{div} u_{0} & =\left(\nabla \varphi_{0} \mid u\right) & & \text { in } \Omega \backslash \Sigma, \\
u_{0} & =0 & & \text { on } \partial \Omega, \\
u_{0}(0) & =0 & & \text { in } \Omega .
\end{aligned}
$$

For $k=N+l(l=1, \ldots, m)$ we have the Cauchy problem of the Stokes equation with parameters $\rho_{1}, \mu_{1}(x)$, i.e.,

$$
\begin{aligned}
\rho_{1} \partial_{t} u_{N+l}-\mu_{1}(x) \Delta u_{N+l}+\nabla \pi_{N+l} & =F_{N+l}(u, \pi) & & \text { in } \mathbb{R}^{n}, \\
\operatorname{div} u_{N+l} & =\left(\nabla \varphi_{N+l} \mid u\right) & & \text { in } \mathbb{R}^{n}, \\
u_{N+l}(0) & =0 & & \text { in } \mathbb{R}^{n} .
\end{aligned}
$$

Though the right members $G_{u_{k}}(h), G_{g_{k}}(u, h), G_{h_{k}}(u), F_{h_{k}}(h)$ have more time regularity than the corresponding data class, the terms $\left(\nabla \varphi_{k}\right) \pi$ in $F_{k}(u, \pi)$ and $\left(\nabla \varphi_{k} \mid u\right)$ unfortunately do not have this property. In order to remove this difficulty, we have to decompose the problem. Here is one major change compared to the 
construction in [14. Consider the following problem for the functions $\phi_{k}, \psi_{k}$.

$$
\begin{array}{rlrl}
\Delta \partial_{t} \phi_{k} & =\partial_{t} u \cdot \nabla \varphi_{k}=\operatorname{div}\left(\partial_{t} u \varphi_{k}\right) & & \text { in } \mathbb{R}^{n} \backslash \Sigma_{k}, \\
\llbracket \partial_{\nu} \partial_{t} \phi_{k} \rrbracket & =\llbracket \partial_{t} u \cdot \nu \varphi_{k} \rrbracket & \text { on } \Sigma_{k}, \\
\Delta \psi_{k} & =\operatorname{div} F_{k} & & \text { in } \mathbb{R}^{n} \backslash \Sigma_{k}, \\
\llbracket \partial_{\nu} \psi_{k} \rrbracket & =\llbracket F_{k} \cdot \nu \rrbracket & & \text { on } \Sigma_{k}, \\
\llbracket \psi_{k}-\rho \partial_{t} \phi_{k} \rrbracket & =0 & & \text { on } \Sigma_{k}, \\
\llbracket \psi_{k} / \rho-\partial_{t} \phi_{k} \rrbracket & =0 & & \text { on } \Sigma_{k},
\end{array}
$$

(7.5) is an elliptic system for $\left(\partial_{t} \phi_{k}, \psi_{k}\right)$ satisfying the Lopatinskii-Shapiro condition, it is uniquely solvable and its solution satisfies $\psi_{k}, \partial_{t} \phi_{k} \in L_{p}\left(J ; \dot{H}_{p}^{1}\left(\mathbb{R}^{n} \backslash \Sigma_{k}\right)\right)$. Hence with $\phi_{k}(0)=0$ we obtain by a time integration $\phi_{k} \in H_{p}^{1}\left(J ; \dot{H}_{p}^{1}\left(\mathbb{R}^{n} \backslash \Sigma_{k}\right)\right)$. Thanks to $\Delta \phi_{k}=\left(\nabla \varphi_{k} \mid u\right)$ in $\mathbb{R}^{n} \backslash \Sigma_{k}$ we conclude that $\phi_{k} \in L_{p}\left(J ; \dot{H}_{p}^{4}\left(\mathbb{R}^{n} \backslash \Sigma_{k}\right)\right)$, which implies

$$
\left.\nabla \phi_{k} \in H_{p}^{1}\left(J ; L_{p}\left(\mathbb{R}^{n}\right)\right) \cap L_{p}\left(J ; H_{p}^{3}\left(\mathbb{R}^{n} \backslash \Sigma_{k}\right)\right), \quad \nabla \psi_{k} \in L_{p}\left(J ; L_{p}\left(\mathbb{R}^{n}\right)\right)\right) .
$$

Defining

$$
\tilde{u}_{k}=u_{k}-\nabla \phi_{k}, \quad \tilde{F}_{k}(u, \pi)=F_{k}(u, \pi)-\nabla \psi_{k},
$$

we see that $\operatorname{div} \tilde{F}_{k}(u, \pi)=0$ and $\operatorname{div} \tilde{u}_{k}=0$ in $\mathbb{R}^{n} \backslash \Sigma_{k}$, as well as

$$
\left(\llbracket \tilde{F}_{k}(u, \pi) \rrbracket \mid \nu\right)=\left(\llbracket \tilde{u}_{k} \rrbracket \mid \nu\right)=0 \quad \text { on } \Sigma_{k} .
$$

Next we define

$$
\tilde{\pi}_{k}=\pi_{k}-\psi_{k}+\rho \partial_{t} \phi_{k}-\mu(x) \Delta \phi_{k}
$$

and observe that

$$
\begin{aligned}
& \llbracket \tilde{\pi}_{k} \rrbracket=\llbracket \pi_{k} \rrbracket-\llbracket \mu(x) \Delta \phi_{k} \rrbracket=\llbracket \pi_{k} \rrbracket-\llbracket \mu(x)\left(\nabla \phi_{k} \mid u\right) \rrbracket \\
& \llbracket \tilde{\pi}_{k} / \rho \rrbracket=\llbracket \pi_{k} / \rho \rrbracket-\llbracket \mu(x) \Delta \phi_{k} / \rho \rrbracket=\llbracket \pi_{k} / \rho \rrbracket-\llbracket \mu(x)\left(\nabla \phi_{k} \mid u\right) / \rho \rrbracket
\end{aligned}
$$

on $\Sigma_{k}$ by construction. Now $\tilde{z}_{k}=\left(\tilde{u}_{k}, \tilde{\pi}_{k}, \llbracket \tilde{\pi}_{k} \rrbracket, h_{k}\right)$ satisfies the problem

$$
\begin{array}{lr}
\rho \partial_{t} \tilde{u}_{k}-\mu(x) \Delta \tilde{u}_{k}+\nabla \tilde{\pi}_{k}=\tilde{F}_{k}(u, \pi) & \text { in } \mathbb{R}^{n} \backslash \Sigma_{k}, \\
\operatorname{div} \tilde{u}_{k}=0 & \text { in } \mathbb{R}^{n} \backslash \Sigma_{k}, \\
P_{\Sigma_{k}} \llbracket \tilde{u}_{k} \rrbracket+c(t, x) \nabla_{\Sigma_{k}} h_{k}=\varphi_{k} P_{\Sigma_{k}} g_{u}+G_{u_{k}}(h) & \text { on } \Sigma_{k}, \\
\llbracket-\mu(x)\left(\nabla \tilde{u}_{k}+\left[\nabla \tilde{u}_{k}\right]^{\top}\right)+\tilde{\pi}_{k} \rrbracket \nu-\sigma\left(\Delta_{\Sigma} h_{k}\right) \nu=\varphi_{k} g_{k}+\tilde{G}_{g_{k}}(u, h) & \text { on } \Sigma_{k}, \\
-\llbracket \mu(x)\left(\nabla \tilde{u}_{k}+\left[\nabla \tilde{u}_{k}\right]^{\top}\right) \nu \cdot \nu / \rho \rrbracket+\llbracket \tilde{\pi}_{k} / \rho \rrbracket=\varphi_{k} g_{h}+\tilde{G}_{h_{k}}(u) & \text { on } \Sigma_{k}, \\
\partial_{t} h_{k}-\llbracket \rho \tilde{u}_{k} \cdot \nu \rrbracket / \llbracket \rho \rrbracket+b(t, x) \cdot \nabla_{\Sigma_{k}} h_{k} / \llbracket \rho \rrbracket=\varphi_{k} f_{h}+\tilde{F}_{h_{k}}(h) & \text { on } \Sigma_{k}, \\
u_{k}(0)=0 & \text { in } \mathbb{R}^{n} \backslash \Sigma_{k}, \\
h_{k}(0)=0 & \text { on } \Sigma_{k},
\end{array}
$$


where

$$
\begin{aligned}
\tilde{G}_{g_{k}}(u, h) & =G_{g_{k}}(u, h)+2 \llbracket \mu(x) \nabla^{2} \phi_{k} \rrbracket \nu_{\Sigma_{k}}-\llbracket \mu(x)\left(\nabla \varphi_{k} \mid u\right) \rrbracket \nu_{\Sigma_{k}}, \\
\tilde{G}_{h_{k}}(u) & =G_{h_{k}}(u)+2 \llbracket \mu(x) \nabla^{2} \phi_{k} / \rho \rrbracket-\llbracket \mu(x)\left(\nabla \varphi_{k} \mid u\right) \nu_{\Sigma_{k}} \cdot \nu_{\Sigma_{k}} / \rho \rrbracket \\
\tilde{F}_{h_{k}}(h) & =F_{h_{k}}(h)+\llbracket \rho \partial_{\nu_{k}} \phi_{k} \rrbracket / \llbracket \rho \rrbracket .
\end{aligned}
$$

Now we know that the data in (7.7) satisfy the assumption of Corollary 3.2 in 14. Therefore the solution $\tilde{\pi}_{k}$ of (7.7) has more time regularity, we have $\bar{\pi}_{k} \in$ ${ }_{0} H_{p}^{\alpha}\left(J ; L_{p}(\Omega)\right)$ for each $\alpha \in(0,1 / 2-1 / 2 p)$. Rewrite (7.7) abstractly as

$$
L_{k} \tilde{z}_{k}=H_{k}+B_{k} z \text {. }
$$

Then by the same argument as in [14] we obtain

$$
\left\|z_{k}\right\|_{\mathbb{E}} \leq C\left(\left\|H_{k}\right\|_{\mathbb{F}}+a^{\gamma}\|z\|_{\mathbb{E}}\right) .
$$

Summing over all $k, z=\sum_{k=1}^{N+m} z_{k}$ yields

$$
\|z\|_{\mathbb{E}} \leq C\left(\|H\|_{\mathbb{F}}+a^{\gamma}\|z\|_{\mathbb{E}}\right) .
$$

Therefore choosing the length $a$ of the time interval small enough, we obtain the a priori estimate

$$
\|z\|_{\mathbb{E}} \leq C\|H\|_{\mathbb{F}} .
$$

Since the problem under consideration is time invariant, repeating this argument finitely many times, we may conclude that the operator $L:{ }_{0} \mathbb{E} \rightarrow{ }_{0} \mathbb{F}$ which maps solutions to their data is injective and has a closed range, i.e., $L$ is a semi-Fredholm operator.

It remains to prove surjectivity of $L$. For this we employ the continuation method for semi-Fredholm operators, which states that the Fredholm index remains constant under homotopies $L(\lambda)$, as long as the ranges of $L(\lambda)$ stay closed. For this purpose, we introduce a first continuation parameter $\alpha \in[0,1]$ by replacing the 7 th equation of (3.4) into

$$
\partial_{t} h+\alpha\left(-\Delta_{\Sigma}\right)^{\frac{1}{2}} h-(1-\alpha)\left(\llbracket \rho u \cdot \nu \rrbracket / \llbracket \rho \rrbracket+b(t, x) \cdot \nabla_{\Sigma} h / \llbracket \rho \rrbracket\right)=f_{h} \quad \text { on } \Sigma .
$$

With minor modifications, the analysis in subsection 7.1 shows that the corresponding problem is well-posed for each $\alpha \in[0,1]$ in the case of a flat interface with bounds independent of $\alpha \in[0,1]$. Therefore the same is true for bent interfaces and then by the above estimates also for a general geometry. Thus we only need to consider the case $\alpha=1$.

To prove surjectivity in this case, note that the equation for $h$ is independent from those for $u$ and $\pi$, and it is uniquely solvable in the right regularity class because of maximal regularity for the Laplace-Beltrami operator. So we may set now $h=0$.

Next we introduce a second continuation parameter $\beta \in[0,1]$ by

$$
P_{\Sigma} u_{2}=\beta P_{\Sigma} u_{1}+P_{\Sigma} g_{u}, \quad-\beta P_{\Sigma} T_{2}(u, \pi) \nu=-P_{\Sigma} T_{1}(u, \pi) \nu=P_{\Sigma} g
$$

with $T(u, \pi)=\mu(x)\left(\nabla u+[\nabla u]^{\top}\right)-\pi$. 
Again, we can prove that the a priori estimates are uniform for $\beta \in[0,1]$. The remaining problem for $\beta=0$ decouples into a one-phase Stokes problem with mixed Dirichlet-Neumann boundary condition in $\Omega_{2}$, Dirichlet condition on $\partial \Omega$ and so-called outflow conditions on $\Sigma$, and a one-phase Stokes problem with pure Neumann boundary condition in $\Omega_{1}$. According to 4 these are well-known to be solvable. This shows that we have surjectivity in the case $\alpha=1$ and $\beta=0$, hence by the continuation method also for $\alpha=0$ and $\beta=1$. The proof of Theorem 3.1 is now complete.

\section{ApPEndiX}

To save space, we follow here the derivation and notation in 23], Section 4.2.

(a) The symbol of $S_{\lambda}^{11}$. To obtain the algebraic system for the symbol of $\left[S_{\lambda}^{11}\right]^{-1}$ we set $g_{2}=0$ and let $k$ be given. Then (4.9) remains valid as well as the formulas for $a_{1}, a_{2}$. For $\alpha_{1}, \alpha_{2}$ we have here the equations

$$
\begin{aligned}
& \frac{\rho_{2} \sqrt{\mu_{2}}}{\omega_{2}} \beta_{2}+\frac{\rho_{1} \sqrt{\mu_{1}}}{\omega_{1}} \beta_{1}+\left(\rho_{1} \alpha_{1}+\rho_{2} \alpha_{2}\right)|\xi|=\llbracket \rho \rrbracket \hat{k} \\
& \frac{2}{\rho_{2}}\left(\mu_{2} \beta_{2}+\mu_{2} \alpha_{2}|\xi|^{2}\right)+\lambda \alpha_{2}-\frac{2}{\rho_{1}}\left(\mu_{1} \beta_{1}+\mu_{1} \alpha_{1}|\xi|^{2}\right)-\lambda \alpha_{1}=0 .
\end{aligned}
$$

Inserting $\beta_{k}$ from $(4.9$,$) this system becomes$

$$
\begin{aligned}
p_{1} \alpha_{1}-p_{2} \alpha_{2} & =0, \\
q_{1} \alpha_{1}+q_{2} \alpha_{2} & =\llbracket \rho \rrbracket k,
\end{aligned}
$$

where

$$
\begin{aligned}
& p_{1}=\rho_{1} \lambda\left[\frac{1}{\rho_{1}}-2 \llbracket \mu / \rho \rrbracket \frac{1}{\gamma(z) \omega_{1}(z)} \frac{\omega_{1}(z)-1}{\omega_{1}(z)+1}\right]=: \rho_{1} \lambda p_{1}^{0}, \\
& p_{2}=\rho_{2} \lambda\left[\frac{1}{\rho_{2}}+2 \llbracket \mu / \rho \rrbracket \frac{1}{\gamma(z) \omega_{2}(z)} \frac{\omega_{2}(z)-1}{\omega_{2}(z)+1}\right]=: \rho_{2} \lambda p_{2}^{0},
\end{aligned}
$$

and

$$
\begin{aligned}
& q_{1}=\frac{\rho_{1} \lambda}{|\xi| \gamma(z)}\left[\frac{\rho_{1}}{\omega_{1}(z)}+\frac{\rho_{1} \mu_{2} \gamma_{2}(z)}{\mu_{1} \omega_{1}(z)\left(\omega_{1}(z)+1\right)}+\frac{\rho_{2}}{\omega_{1}(z) \omega_{2}(z)} \frac{\omega_{1}(z)-1}{\omega_{1}(z)+1}\right]=: \frac{\rho_{1} \lambda}{|\xi| \gamma(z)^{2}} q_{1}^{0} \\
& q_{2}=\frac{\rho_{2} \lambda}{|\xi| \gamma(z)}\left[\frac{\rho_{2}}{\omega_{2}(z)}+\frac{\rho_{2} \mu_{1} \gamma_{1}(z)}{\mu_{2} \omega_{2}(z)\left(\omega_{2}(z)+1\right)}+\frac{\rho_{1}}{\omega_{1}(z) \omega_{2}(z)} \frac{\omega_{2}(z)-1}{\omega_{2}(z)+1}\right]=: \frac{\rho_{2} \lambda}{|\xi| \gamma(z)^{2}} q_{2}^{0},
\end{aligned}
$$

where the scaling $z=\lambda /|\xi|^{2}$ is employed, and recall

$$
\omega_{k}(z)=\sqrt{1+\rho_{k} z / \mu_{k}}, \quad \gamma_{k}(z)=\omega_{k}(z)+1 / \omega_{k}(z), \quad \gamma(z)=\mu_{1} \gamma_{1}(z)+\mu_{2} \gamma_{2}(z) .
$$

This yields the transformed interface pressures

$$
\begin{aligned}
& \hat{\pi}_{1}=\rho_{1} \lambda \alpha_{1}=\llbracket \rho \rrbracket \frac{p_{2}^{0}}{p_{1}^{0} q_{2}^{0}+p_{2}^{0} q_{1}^{0}}|\xi| \gamma(z)^{2} \hat{k}, \\
& \hat{\pi}_{2}=\rho_{2} \lambda \alpha_{2}=\llbracket \rho \rrbracket \frac{p_{1}^{0}}{p_{1}^{0} q_{2}^{0}+p_{2}^{0} q_{1}^{0}}|\xi| \gamma(z)^{2} \hat{k} .
\end{aligned}
$$

Note that $p_{k}^{0}, q_{0}^{k}$ are holomorphic in $\mathbb{C} \backslash(-\infty,-\eta], \eta=\min \left\{\mu_{k} / \rho_{k}\right\}>0$, and we have

$$
p_{k}^{0}(0)=1 / \rho_{k}, \quad q_{k}^{0}(0)=2\left(\mu_{1}+\mu_{2}\right)^{2} \rho_{k} / \mu_{k},
$$


and

$$
p_{k}^{0}(\infty)=1 / \rho_{k}, \quad q_{k}^{0}(\infty)=\left(\rho_{1}+\rho_{2}\right) \sqrt{\rho_{k} \mu_{k}}
$$

Therefore $p_{k}^{0}, q_{k}^{0}$ are holomorphic and bounded on $\Sigma_{\pi / 2+\varepsilon} \cup B_{\varepsilon}(0)$, for small $\varepsilon>0$.

So we need to show that the Lopatinskii-Determinant $r^{0}(z):=p_{1}^{0}(z) q_{2}^{0}(z)+p_{2}^{0}(z) q_{1}^{0}(z)$ has no zeros in $\operatorname{Re} z \geq 0$. After some tedious algebra, expanding and collecting terms, we obtain the factorization $r^{0}(z)=r_{1}^{0}(z) r_{2}^{0}(z)$, where

$$
r_{1}^{0}(z)=\gamma(z) /\left[\omega_{1}(z) \omega_{2}(z)\left(\omega_{1}(z)+1\right)\left(\omega_{2}(z)+1\right)\right],
$$

and

$$
\begin{aligned}
r_{2}^{0}(z) & =\left(\frac{\rho_{2}}{\rho_{1}} \omega_{1}(z)+\frac{\rho_{1}}{\rho_{2}} \omega_{2}(z)\right)\left(\omega_{1}(z)+1\right)\left(\omega_{2}(z)+1\right)\left(\omega_{1}(z)+1\right)\left(\omega_{2}(z)+1\right) \\
& +2\left(\omega_{1}(z)-1\right)\left(\omega_{2}(z)-1\right)+2 \frac{\mu_{2} \rho_{1}}{\mu_{1} \rho_{2}}\left(\omega_{2}(z)-1\right)+2 \frac{\mu_{1} \rho_{2}}{\mu_{2} \rho_{1}}\left(\omega_{1}(z)-1\right) \\
& +\frac{\mu_{2} \rho_{1}}{\mu_{1} \rho_{2}}\left(\omega_{2}^{2}(z)+1\right)\left(\omega_{2}(z)+1\right)+\frac{\mu_{1} \rho_{2}}{\mu_{2} \rho_{1}} \gamma_{1}(z) \omega_{1}(z)\left(\omega_{1}(z)+1\right)
\end{aligned}
$$

Obviously, $r_{1}^{0}(z)$ has no zeros in $\left.\mathbb{C} \backslash(-\infty, 0]\right)$; so we only have to look at $r_{2}^{0}(z)$. One easily checks that each summand of $r_{2}^{0}(z)$ has nonnegative imaginary part, provided $z \in \mathbb{C}$ is such that $\operatorname{Re} z, \operatorname{Im} z \geq 0$, so $r_{2}^{0}(z)$ can only be zero if each summand is zero. But this is not possible, as already the first term shows.

(b) The symbol of $S_{\lambda}^{22}$. To obtain the algebraic system for the symbol of $\left[S_{\lambda}^{22}\right]^{-1}$ we set $g_{1}=0$ and let $j$ be given. Then (4.9) remains valid as well as the formulas for $a_{1}, a_{2}$. For $\alpha_{1}, \alpha_{2}$ we have here the equations

$$
\begin{aligned}
& 2 \mu_{2}\left(\beta_{2}+|\xi|^{2} \alpha_{2}\right)+ \rho_{2} \lambda \alpha_{2}-2 \mu_{1}\left(\beta_{1}+|\xi|^{2} \alpha_{1}\right)+\rho_{1} \lambda \alpha_{1}=0, \\
& \frac{\sqrt{\mu_{2}}}{\omega_{2}} \beta_{2}+|\xi| \alpha_{2}+\frac{\sqrt{\mu_{1}}}{\omega_{1}} \beta_{1}+|\xi| \alpha_{1}=\llbracket 1 / \rho \rrbracket \hat{j} .
\end{aligned}
$$

Inserting $\beta_{k}$ from (4.9) this system becomes

$$
\begin{aligned}
p_{1} \alpha_{1}-p_{2} \alpha_{2} & =0, \\
q_{1} \alpha_{1}+q_{2} \alpha_{2} & =\llbracket 1 / \rho \rrbracket j,
\end{aligned}
$$

where

$$
\begin{aligned}
& p_{1}=\rho_{1} \lambda\left[1+2 \llbracket \mu \rrbracket \frac{1}{\gamma(z) \omega_{1}(z)} \frac{\omega_{1}(z)-1}{\omega_{1}(z)+1}\right]=: \rho_{1} \lambda p_{1}^{0}, \\
& p_{2}=\rho_{2} \lambda\left[1-2 \llbracket \mu \rrbracket \frac{1}{\gamma(z) \omega_{2}(z)} \frac{\omega_{2}(z)-1}{\omega_{2}(z)+1}\right]=: \rho_{2} \lambda p_{2}^{0},
\end{aligned}
$$

and

$$
\begin{aligned}
& q_{1}=\frac{\rho_{1} \lambda}{|\xi| \omega_{1}(z) \gamma(z)}\left[1+\frac{1}{\omega_{2}(z)} \frac{\omega_{1}(z)-1}{\omega_{1}(z)+1}+\frac{\gamma_{2}(z)}{\mu_{1}\left(\omega_{1}(z)+1\right)}\right]=: \frac{\rho_{1} \lambda}{|\xi| \gamma(z)^{2}} q_{1}^{0}, \\
& q_{2}=\frac{\rho_{2} \lambda}{|\xi| \omega_{2}(z) \gamma(z)}\left[1+\frac{1}{\omega_{1}(z)} \frac{\omega_{2}(z)-1}{\omega_{2}(z)+1}+\frac{\gamma_{1}(z)}{\mu_{2}\left(\omega_{2}(z)+1\right)}\right]=: \frac{\rho_{2} \lambda}{|\xi| \gamma(z)^{2}} q_{2}^{0} .
\end{aligned}
$$

This yields

$$
\begin{aligned}
& \hat{\pi}_{1}=\rho_{1} \lambda \alpha_{1}=\llbracket 1 / \rho \rrbracket \frac{p_{2}^{0}}{p_{1}^{0} q_{2}^{0}+p_{2}^{0} q_{1}^{0}}|\xi| \gamma(z)^{2} \hat{j}, \\
& \hat{\pi}_{2}=\rho_{2} \lambda \alpha_{2}=\llbracket 1 / \rho \rrbracket \frac{p_{1}^{0}}{p_{1}^{0} q_{2}^{0}+p_{2}^{0} q_{1}^{0}}|\xi| \gamma(z)^{2} \hat{j} .
\end{aligned}
$$


As in (a) the functions $p_{k}^{0}, q_{k}^{0}$ are holomorphic in $\mathbb{C} \backslash(-\infty,-\eta], \eta=\min \left\{\mu_{k} / \rho_{k}\right\}>0$, and we have

and

$$
p_{k}^{0}(0)=1, \quad q_{k}^{0}(0)=2\left(\mu_{1}+\mu_{2}\right)^{2} / \mu_{k}
$$

$$
p_{k}^{0}(\infty)=1, \quad q_{k}^{0}(\infty)=\left(\sqrt{\rho_{1} \mu_{1}}+\sqrt{\rho_{2} \mu_{2}}\right)^{2} \rho_{k} .
$$

Therefore $p_{k}^{0}, q_{k}^{0}$ are holomorphic and bounded on $\Sigma_{\pi / 2+\varepsilon} \cap B_{\varepsilon}(0)$, for small $\varepsilon>0$.

So we need to show that the Lopatinskii-determinant $r^{0}(z):=p_{1}^{0}(z) q_{2}^{0}(z)+p_{2}^{0}(z) q_{1}^{0}(z)$ has no zeros in $\operatorname{Re} z \geq 0$. After another tedious algebra, expanding and collecting terms, we obtain as in (a) the factorization $r^{0}(z)=r_{1}^{0}(z) r_{2}^{0}(z)$, where

$$
r_{1}^{0}(z)=\gamma(z) /\left[\omega_{1}(z) \omega_{2}(z)\left(\omega_{1}(z)+1\right)\left(\omega_{2}(z)+1\right)\right],
$$

and

$$
\begin{aligned}
r_{2}^{0}(z) & =\left(\omega_{1}(z)+\omega_{2}(z)\right)\left(\omega_{1}(z)+1\right)\left(\omega_{2}(z)+1\right)+2\left(\omega_{1}(z)-1\right)\left(\omega_{2}(z)-1\right) \\
& +\frac{\mu_{2}}{\mu_{1}}\left[2\left(\omega_{2}(z)-1\right)+\left(\omega_{2}^{2}(z)+1\right)\left(\omega_{2}(z)+1\right)\right] \\
& +\frac{\mu_{1}}{\mu_{2}}\left[2\left(\omega_{1}(z)-1\right)+\left(\omega_{1}^{2}(z)+1\right)\left(\omega_{1}(z)+1\right)\right] .
\end{aligned}
$$

Obviously, $r_{1}^{0}(z)$ has no zeros in $\left.\mathbb{C} \backslash(-\infty, 0]\right)$; so we only have to look at $r_{2}^{0}(z)$. One easily checks that each summand of $r_{2}^{0}(z)$ has nonnegative imaginary part, provided $z \in \mathbb{C}$ is such that $\operatorname{Re} z, \operatorname{Im} z \geq 0$, so $r_{2}^{0}(z)$ can only be zero if each summand is zero. But this is not possible, as already the first term shows.

(c) The symbol of $s(\lambda, \tau)$. We calculate the symbol $s(\lambda, \tau)$ defined by (7.2) in the same way as Sec.4-3 in 23]. It is enough to seek the solution of the problem

$$
\begin{aligned}
\hat{v}_{2}(0)-\hat{v}_{1}(0) & =-c_{0} i \xi \hat{h}, \\
\mu_{2}\left(\partial_{y} \hat{v}_{2}(0)+i \xi \hat{w}_{2}(0)\right)-\mu_{1}\left(\partial_{y} \hat{v}_{1}(0)+i \xi \hat{w}_{1}(0)\right) & =0, \\
-2 \mu_{2} \partial_{y} \hat{w}_{2}(0)+\hat{\pi}_{2}(0)+2 \mu_{1} \partial_{y} \hat{w}_{1}(0)-\hat{\pi}_{1}(0) & =0, \\
-2\left(\mu_{2} / \rho_{2}\right) \partial_{y} \hat{w}_{2}(0)+\hat{\pi}_{2}(0) / \rho_{2}+2\left(\mu_{1} / \rho_{2}\right) \partial_{y} \hat{w}_{1}(0)-\hat{\pi}_{1}(0) / \rho_{2} & =0 .
\end{aligned}
$$

Multiplying the 1 st and the 2 nd equations by $i \xi$, and setting $\beta_{j}=i \xi \cdot a_{j}$ for $a_{j} \in \mathbb{C}^{n-1}$, $j=1,2$, we have

$$
\begin{aligned}
\beta_{2}-\beta_{1} & =-|\xi|^{2}\left\{\left(\alpha_{2}-\alpha_{1}\right)-c_{0} \hat{h}\right\} \\
\sqrt{\mu_{2}} \omega_{2} \beta_{2}+\sqrt{\mu_{1}} \omega_{1} \beta_{1} & =-|\xi|^{2}\left(2|\xi|\left(\mu_{1} \alpha_{1}+\mu_{2} \alpha_{2}\right)+\left(\mu_{1} \sqrt{\mu_{1}} / \omega_{1}\right) \beta_{1}+\left(\mu_{2} \sqrt{\mu_{2}} / \omega_{2}\right) \beta_{2}\right) .
\end{aligned}
$$

Combining the 3 rd and the 4 th equation of (8.5), we obtain

$$
\alpha_{j}=-2 \mu_{j} \beta_{j} /\left(\omega_{j}^{2}+\mu_{j}|\xi|^{2}\right)
$$

Substituting the formula into (8.6) and using the scaling $\omega_{j}=\sqrt{\mu_{j}}|\xi| \omega_{j}(z), \gamma_{j}=$ $\mu_{j}|\xi| \gamma_{j}(z)$, we solve the system for $\beta_{j}$

$$
\left(\begin{array}{cc}
-1+2 /\left(\omega_{1}(z)^{2}+1\right) & 1-2 /\left(\omega_{2}(z)^{2}+1\right) \\
\mu_{1}\left\{\gamma_{1}(z)-4 /\left(\omega_{1}(z)^{2}+1\right)\right\} & \mu_{2}\left\{\gamma_{2}(z)-4 /\left(\omega_{2}(z)^{2}+1\right)\right\}
\end{array}\right)\left(\begin{array}{c}
\beta_{1} \\
\beta_{2}
\end{array}\right)=\left(\begin{array}{c}
c_{0} \tau^{2} \hat{h} \\
0
\end{array}\right),
$$

where we set $\tau=|\xi|$ and $z=\lambda / \tau^{2}$. Therefore substituting the $\beta_{j}$ and $\alpha_{j}$ by (8.7) into

we obtain (7.2).

$$
-\frac{\llbracket \rho w(0) \rrbracket}{\llbracket \rho \rrbracket}=-\frac{\left(\rho_{1} \alpha_{1}+\rho_{2} \alpha_{2}\right) \tau+\left(\rho_{2} / \tau \omega_{2}\right) \beta_{2}+\left(\rho_{1} / \tau \omega_{1}\right) \beta_{1}}{\llbracket \rho \rrbracket},
$$




\section{REFERENCES}

[1] H. Abels, M. Wilke, Well-posedness and qualitative behaviour of solutions for a two-phase Navier-Stokes-Mullins-Sekerka system. Interfaces and Free Boundaries, to appear 2013.

[2] D.M. Anderson, P. Cermelli, E. Fried, M.E. Gurtin, G.B. McFadden, General dynamical sharp-interface conditions for phase transformations in viscous heat-conducting fluids. $J$. Fluid Mech. 581 (2007), 323-370.

[3] D. Bothe and J. Prüss, $L_{p}$-Theory for a class of Non-Newtonian fluids. SIAM J. Math. Anal. 39 (2007), 379-421.

[4] D. Bothe, M. Köhne and J. Prüss, On a class of energy preserving boundray conditions for incompressible Newtonian flows. submitted 2012. arXiv:1207.0707

[5] E. DiBenedetto, A. Friedman, Conduction-convection problems with change of phase, $J$. Differential Equations 62 (1986), no. 2, 129-185.

[6] E. DiBenedetto, M. O'Leary, Three-dimensional conduction-convection problems with change of phase, Arch. Rational Mech. Anal. 123 (1993), no. 2, 99-116.

[7] R. Denk, M. Hieber, and J. Prüss, $\mathcal{R}$-boundedness, Fourier multipliers, and problems of elliptic and parabolic type, AMS Memoirs 788, Providence, R.I. (2003).

[8] R. Denk, M. Hieber, J.Prüss, Optimal $L^{p}-L^{q}$-estimates for parabolic boundary value problems with inhomogeneous data. Math. Z. 257 (2007), no. 1, 193-224.

[9] K.-H. Hoffmann, V.N. Starovoitov, The Stefan problem with surface tension and convection in Stokes fluid, Adv. Math. Sci. Appl. 8 (1998), no. 1, 173-183.

[10] K.-H. Hoffmann, V.N. Starovoitov, Phase transitions of liquid-liquid type with convection, Adv. Math. Sci. Appl. 8 (1998), no. 1, 185-198.

[11] M. Ishii, Thermo-Fluid Dynamic Theory of Two-Phase Flow Collection de la Direction des Études et Recherches D'Électricité d France, Paris 1975.

[12] M. Ishii, H. Takashi Thermo-fluid dynamics of two-phase flow. Springer, New York, 2006.

[13] M. Köhne, J. Prüss, M. Wilke, On quasilinear parabolic evolution equations in weighted $L_{p}$-spaces. J. Evol. Equ. 10, 443-463 (2010).

[14] M. Köhne, J. Prüss, M. Wilke, Qualitative behaviour of solutions for the two-phase NavierStokes equations with surface tension. Math. Annalen to appear 2013.

[15] Y. Kusaka, On a limit problem of the Stefan problem with surface tension in a viscous incompressible fluid flow, Adv. Math. Sci. Appl. 12 (2002), no. 2, 665-683.

[16] Y. Kusaka, A. Tani, On the classical solvability of the Stefan problem in a viscous incompressible fluid flow, SIAM J. Math. Anal. 30 (1999), no. 3, 584-602 (electronic).

[17] Y. Kusaka, A. Tani, Classical solvability of the two-phase Stefan problem in a viscous incompressible fluid flow, Math. Models Methods Appl. Sci. 12 (2002), no. 3, 365-391.

[18] J. Prüss, Maximal regularity for evolution equations in $L_{p}$-spacess. Conf. Sem. Mat. Univ. Bari 285, 1-39 (2003)

[19] J. Prüss, G. Simonett, Maximal regularity for evolution equations in weighted $L_{p}$-spaces. Archiv Math. 82, 415-431 (2004)

[20] J. Prüss, G. Simonett, On the two-phase Navier-Stokes equations with surface tension. Interfaces and Free Boundaries, 12, 311-345 (2010)

[21] J. Prüss, G. Simonett, Analytic solutions for the two-phase Navier-Stokes equations with surface tension. Progress in Nonlinear Differential Equations and their Applications 80, 507-540 (2011).

[22] J. Prüss, Y. Shibata, S. Shimizu, G. Simonett, On well-posedness of incompressible twophase flows with phase transition: The case of equal densities. Evolution Equations 83 Control Theory 1., 171-194 (2012)

[23] J. Prüss, S. Shimizu, On well-posedness of incompressible two-phase flows with phase transition: The case of non-equal densities. J. Evolution Eqns.12 917-941 (2012)

[24] J. Prüss, G. Simonett, On the manifold of closed hypersurfaces in $\mathbb{R}^{n}$. Discrete Cont. Dyn. Systems, to appear 2012. arXiv:1212.6445 
[25] J. Prüss, G. Simonett, R. Zacher, Convergence of solutions to equilibria for nonlinear parabolic problems. J. Diff. Equations 246, 3902-3931 (2009)

[26] J. Prüss, G. Simonett, R. Zacher, Qualitative behaviour of solutions for thermodynamically consistent Stefan problems with surface tension. Arch. Rat. Mech. Anal. to appear 2013

[27] J. Prüss, G. Simonett, R. Zacher, On the qualitative behaviour of incompressible two-phase flows with phase transition: The case of equal densities. Submitted 2012. arXiv:1209.3814

[28] S. Shibata, S. Shimizu, On the $L_{p}-L_{q}$ maximal regularity of the Neumann problem for the Stoke equations in a bounded domain. J. Reine Angew. Math. 615, 157-209 (2008)

Institut für Mathematik, Martin-Luther-Universität Halle-WittenberG, D-06099 Halle, Germany

E-mail address: jan.pruess@mathematik.uni-halle.de

Department of Mathematics, Shizuoka University, 422-8529 Shizuoka, Japan

E-mail address: ssshimi@ipc.shizuoka.ac.jp

Institut für Mathematik, Martin-Luther-Universität Halle-Wittenberg, D-06099 Halle, Germany

E-mail address: mathias.wilke@mathematik.uni-halle.de 